\title{
Effects of Size and Size Distribution on the Magnetic Properties of Maghemite Nanoparticles and Iron-Platinum Core-Shell Nanoparticles
}

\author{
Kelly Pisane
}

Follow this and additional works at: https://researchrepository.wvu.edu/etd

\section{Recommended Citation}

Pisane, Kelly, "Effects of Size and Size Distribution on the Magnetic Properties of Maghemite Nanoparticles and Iron-Platinum Core-Shell Nanoparticles" (2015). Graduate Theses, Dissertations, and Problem Reports. 6423.

https://researchrepository.wvu.edu/etd/6423

This Dissertation is protected by copyright and/or related rights. It has been brought to you by the The Research Repository @ WVU with permission from the rights-holder(s). You are free to use this Dissertation in any way that is permitted by the copyright and related rights legislation that applies to your use. For other uses you must obtain permission from the rights-holder(s) directly, unless additional rights are indicated by a Creative Commons license in the record and/ or on the work itself. This Dissertation has been accepted for inclusion in WVU Graduate Theses, Dissertations, and Problem Reports collection by an authorized administrator of The Research Repository @ WVU.

For more information, please contact researchrepository@mail.wvu.edu. 


\title{
Effects of Size and Size Distribution on the Magnetic Properties of Maghemite Nanoparticles and Iron-Platinum Core-Shell Nanoparticles
}

\section{Kelly Pisane}

\author{
Dissertation submitted \\ to the Eberly College of Arts and Sciences \\ at West Virginia University \\ in partial fulfillment of the requirements for the degree of \\ Doctor of Philosophy in \\ Physics
}

Mohindar Seehra, Ph.D., Chair

David Lederman, Ph.D.

Mikel Holcomb, Ph.D.

Edward Flagg, Ph.D.

Aleksandr Stefaniak, Ph.D.

Department of Physics \& Astronomy

Morgantown, West Virginia

2015

Keywords: $\gamma-\mathrm{Fe}_{2} \mathrm{O}_{3}$, Magnetic Nanoparticles, Core-Shell Nanoparticles, Magnetism, Magnetic

Anisotropy, Interparticle Interactions

Copyright 2015 Kelly L. Pisane 


\section{ABSTRACT \\ Effects of Size and Size Distribution on the Magnetic Properties of Maghemite Nanoparticles and Core-Shell Iron-Platinum Nanoparticles}

\section{Kelly L. Pisane}

Magnetic nanoparticles with large magnetic moments that can be manipulated with an external magnetic field, have potential uses in medicine because their sizes are comparable to biological scales. For such applications it is important to understand how their magnetic properties are affected by their size and size distribution inherently present in magnetic nanoparticles. For this purpose, maghemite $\left(\gamma-\mathrm{Fe}_{2} \mathrm{O}_{3}\right)$ nanoparticles with average diameters of $7.0 \pm 0.8 \mathrm{~nm}, 6.3 \pm 0.6 \mathrm{~nm}, 3.4 \pm 0.8 \mathrm{~nm}$ and $2.5 \pm 0.7 \mathrm{~nm}$ and Fe-Pt core-shell nanoparticles with an approximate core diameter of $2.2 \mathrm{~nm}$ were synthesized and investigated. To aid in the interpretation of the magnetic properties, the structural properties of these nanoparticles were investigated using transmission electron microscopy (TEM), X-ray diffraction (XRD), infrared spectroscopy (FTIR), and thermogravimetric analysis (TGA).

For investigations of the magnetic properties, detailed ac and dc magnetic characterization is presented and discussed in terms of a distribution of particle sizes and magnetic moments. The dc magnetization measurements cover the temperature range from $2 \mathrm{~K}$ to $350 \mathrm{~K}$ and magnetic fields up to 90 kOe. The temperature dependence of the ac susceptibilities, $\chi^{\prime}$ and $\chi^{\prime \prime}$, was measured at various frequencies from $10 \mathrm{~Hz}$ to $5 \mathrm{kHz}$. From the zero field-cooled dc magnetization, the values of blocking temperature $T_{B}$ have been determined and the ac magnetic data was used to determine the contribution of interparticle interactions to the observed blocking temperature for different sized nanoparticles. The measured blocking temperatures of the maghemite nanoparticles are $T_{B}=35 \mathrm{~K}, 42 \mathrm{~K}, 21 \mathrm{~K}$, and $29 \mathrm{~K}$ with contributions from interparticle interactions given in terms of $T_{o}=0 \mathrm{~K}, 11 \mathrm{~K}, 2.5 \mathrm{~K}$, and $12.5 \mathrm{~K}$ for the $7.0 \mathrm{~nm}, 6.3 \mathrm{~nm}, 3.4 \mathrm{~nm}$, and $2.5 \mathrm{~nm}$ samples respectively. From the variation of $T_{B}$ with ac measurement frequency, the anisotropy constants $K_{a}$ determined for the maghemite nanoparticles are: $K_{a}=5.57,7.51,18.57$, and 79.9 in units of $10^{5} \mathrm{erg} / \mathrm{cm}^{3}$ for the $7.0 \mathrm{~nm}, 6.3 \mathrm{~nm}, 3.4 \mathrm{~nm}$, and $2.5 \mathrm{~nm}$ samples with a Néel-Brown attempt frequency of $f_{o}=2.6 \times 10^{10} \mathrm{~Hz}$. The same approach applied to Fe-Pt nanoparticles yields $T_{B}=13 \mathrm{~K}, T_{o}=5 \mathrm{~K}, K_{a}=4.74 \times 10^{6} \mathrm{erg} / \mathrm{cm}^{3}$, and $f_{o}=5.3 \times 10^{10} \mathrm{~Hz}$. For maghemite nanoparticles, the size dependence of the anisotropy shows an increase with decreasing particle diameter consistent with data of other investigators. However this dependence is more rapid than the $1 / D$ behavior typically used to discuss the size dependence of nanoparticle magnetic anisotropy.

The magnetic field dependence of the magnetization of the nanoparticles below their blocking temperature $T_{B}$ indicates negligible coercivity for the $7.0 \mathrm{~nm}, 3.4 \mathrm{~nm}$, and $2.5 \mathrm{~nm}$ maghemite samples. However, for the $6.3 \mathrm{~nm}$ maghemite and the Fe-Pt samples, significant coercivity $H_{C}$ is observed with their magnitudes increasing with decreasing temperatures below $T_{B}$ and reaching 400 Oe and 750 Oe at 2 $\mathrm{K}$, respectively. Above $T_{B}$ the field dependence of the magnetization of all the samples was analyzed in two different ways: in terms of a modified Langevin equation that ignores the distribution of particle diameters and in terms of a lognormal distribution of particle magnetic moments mimicking the size distribution. An important conclusion from this comparison is that the two approaches yield consistent and physically meaningful results as long as the width parameter, $s$, of the log-normal distribution is less than 0.83 .

Another important result from these investigations is the derivation of the Eq. $\Phi=\Phi_{o}[1-$ $\left.\left(T_{o} / T_{B}\right)\right]$ relating the parameter $\Phi$ used to describe the interparticle interaction strength to the VogelFulcher temperature $T_{o}$ in addition to providing the theoretical basis for the experimentally observed $\Phi_{o} \approx 0.11$ to 0.15 . Experimental verification of this relationship is presented using the published data on a variety of nanoparticle systems. 


\section{Acknowledgments}

I take this opportunity to express my gratitude to the people who have supported me through this process:

Thank you Dr. Mohindar Seehra for your support and guidance and for offering to step in as my advisor so that I could complete my PhD. I feel extremely lucky to have had the opportunity to work with you and learn from you.

I thank Dr. Aleksandr Stefaniak for giving me the opportunity to visit NIOSH and learn more about toxicology research and agreeing to serve on my committee. I thank my committee members Dr. Edward Flagg, Dr. Mikel Holcomb, and especially Dr. David Lederman — I really enjoyed getting the opportunity to take classes with you and appreciate all the help you have given me throughout the years.

I would also like to thank the other department staff members who have helped me throughout my time here: Carl Weber, Doug Mathess, JR Raber, George Parnell, Amy Matuga, Vanessa Baker, Vicki \& Hannah Snively. I am especially grateful to Greg \& Sherry Puskar, Viola Bryant, Philip Tucker \& Kim Quedado whose support and camaraderie helped make my last few years in the department happy ones.

To my lab mates (past and present) Vishal Narang, Zhengjun Wang, Sobhit Singh, Usha Geddham \& Phil Tabor, thank you for your support and friendship.

I thank my friends, particularly Stephanie Sears, Josh Miller, Audrey \& Aaron Holsclaw, and Will Booth. A special thanks goes to Jeff Walker for letting me take over his apartment as my dissertation writing station, putting up with my ridiculous hours over the years, and believing in me when I wasn't so sure I could make it.

I thank my family, especial my parents Cindy \& Joe Pisane, for their unwavering belief that I could do this; it meant a great deal to me.

Finally, I acknowledge NSF Grant \#1144676 and the WVU IGERT program for supporting the research presented here. 


\section{Table of Contents}

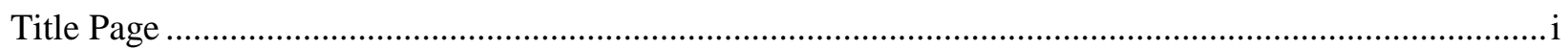

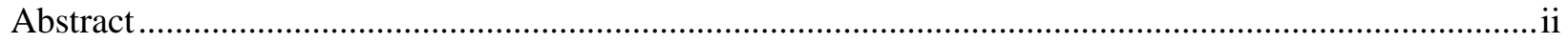

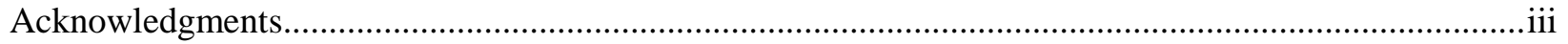

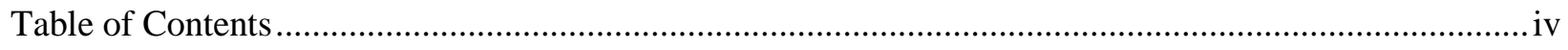

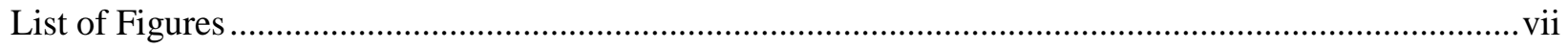

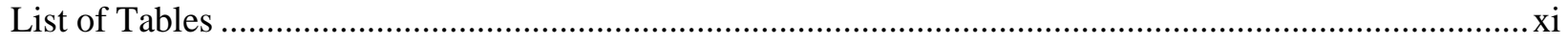

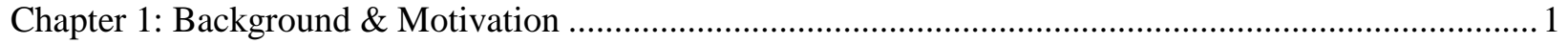

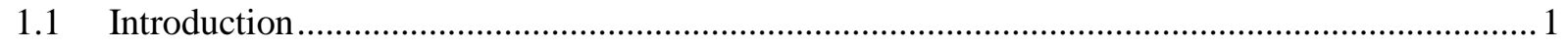

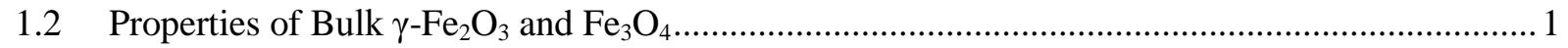

1.3 Previous Studies on $\gamma-\mathrm{Fe}_{2} \mathrm{O}_{3}$ Nanoparticles ....................................................................

1.4 Motivation for this Research......................................................................................

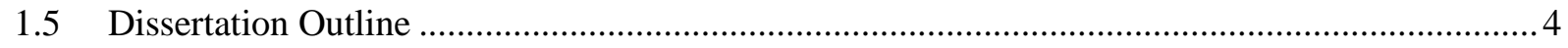

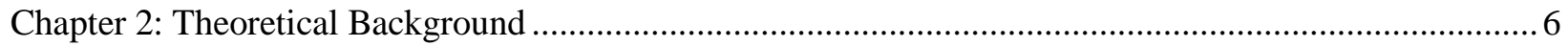

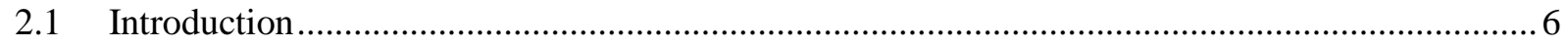

2.2 Temperature \& Magnetic Field Dependence of Magnetization ....................................................6

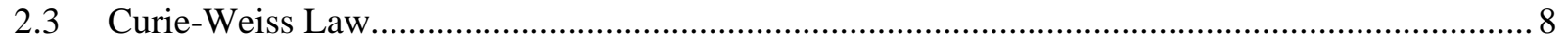

2.4 Exchange Interaction

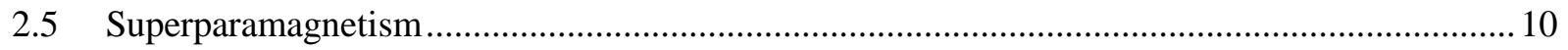

2.5.1 Magnetic Relaxation and Blocking Temperature.............................................................. 11

2.5.2 Effects of Magnetic Field on the Energy Barrier ......................................................... 13

2.6 Magnetic Field Dependence of Magnetization at Temperatures above $T_{B} \ldots \ldots \ldots \ldots \ldots \ldots \ldots \ldots \ldots \ldots \ldots \ldots . . .14$

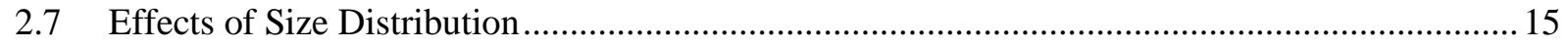

2.8 Temperature and Frequency Dependence of ac Susceptibilities..................................................15

Chapter 3: Synthesis and Structural Characterization of Maghemite Nanoparticles ................................... 18

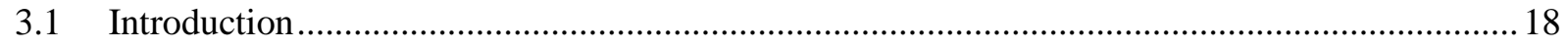

3.2 Synthesis of Maghemite Nanoparticles with Oleic Acid Coating................................................. 18

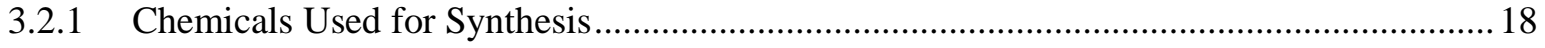

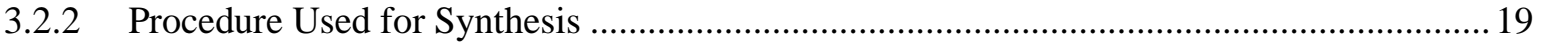

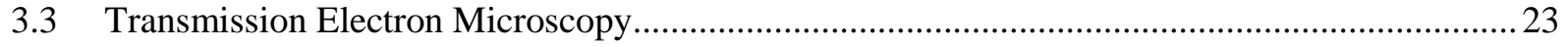

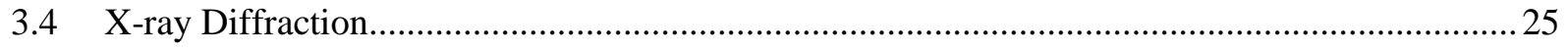

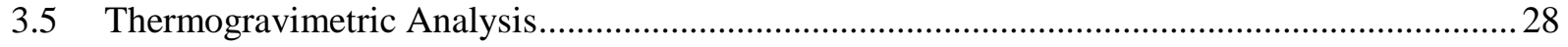

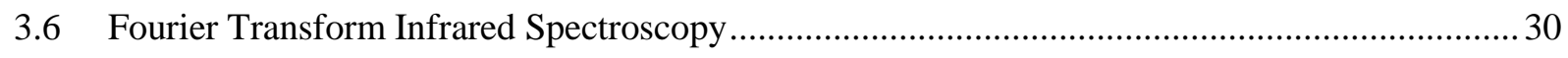




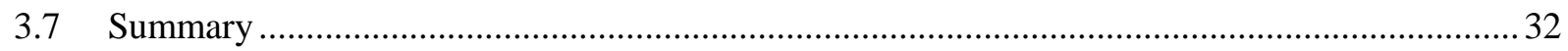

Chapter 4: Magnetic Properties of 7.0 nm Maghemite Nanoparticles ...................................................... 33

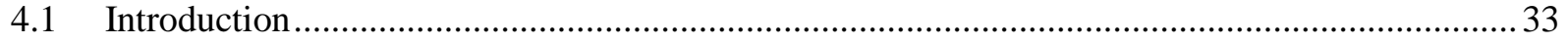

4.2 Procedures for Magnetic Measurements ............................................................................... 33

4.2.1 Preparation and Loading of the Sample for Magnetic Measurements ................................33

4.2.2 Mass and Background Determination .......................................................................... 34

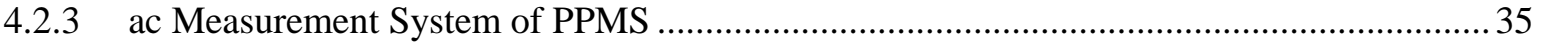

4.2.4 Measurement Programming ...................................................................................... 35

4.2.5 Zero Field-Cooled and Field-Cooled Measurements ........................................................ 36

4.3 Temperature \& Magnetic Field Dependence of Magnetization of the $7.0 \mathrm{~nm} \gamma-\mathrm{Fe}_{2} \mathrm{O}_{3}$ Sample .. 37

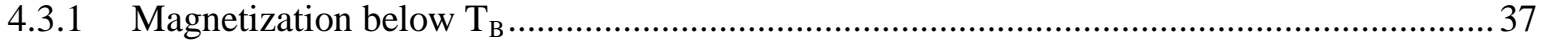

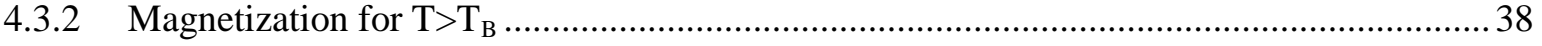

4.3.3 Temperature and Frequency Dependence of ac Susceptibilities ....................................... 39

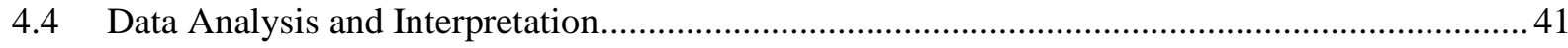

4.4.1 Néel-Brown Relaxation and Interparticle Interaction ................................................. 41

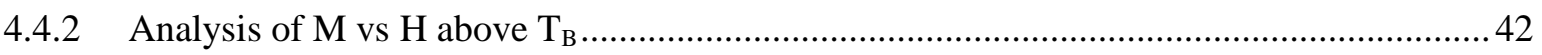

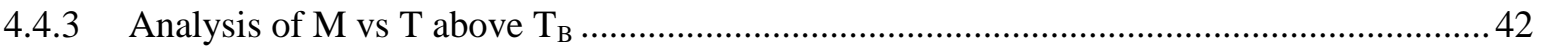

4.4.4 Analysis using a Distribution of Magnetic Moments ......................................................... 44

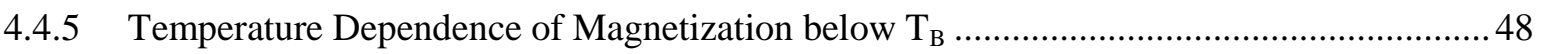

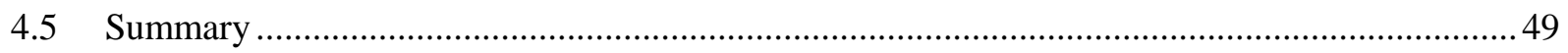

Chapter 5: Size Dependence of the Magnetic Properties of Maghemite Nanoparticles ............................51

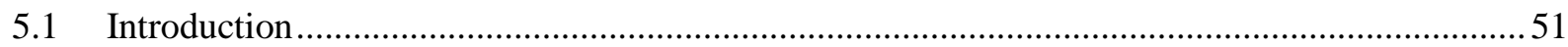

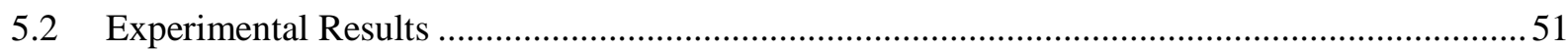

5.2.1 Temperature Dependence of the Magnetization of $\gamma-\mathrm{Fe}_{2} \mathrm{O}_{3}$ Nanoparticles.........................51

5.2.2 Magnetic Field Dependence of dc Magnetization...........................................................52

5.2.3 Temperature and Frequency Dependence of ac Susceptibilities .......................................55

5.3 Analysis, Interpretation, and Discussion of the Results ........................................................57

5.3.1 Size and Frequency Dependence of the Blocking Temperatures ......................................57

5.3.2 Effects of Interparticle Interactions on the Blocking Temperatures ...................................58

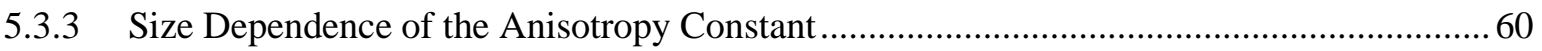

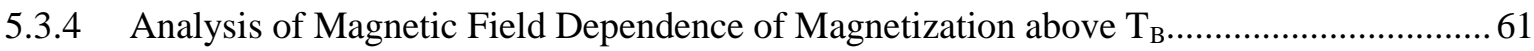

5.3.5 Discussion of Modified Langevin Fit Results ..................................................................6 65

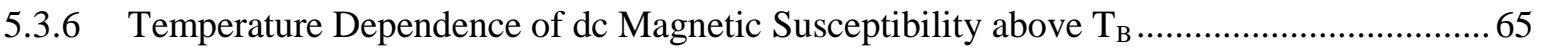

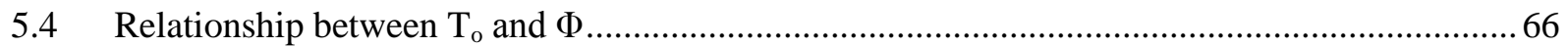


5.5 Summary .68

Chapter 6: Iron-Platinum Core-Shell Nanoparticles: Synthesis, Structural Characterization, and Magnetic Properties .69

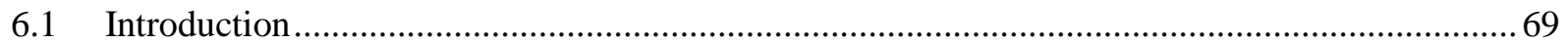

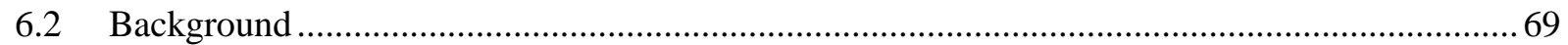

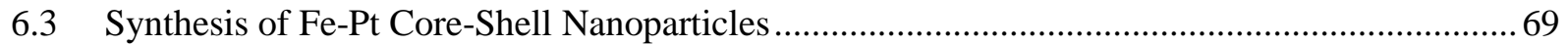

6.3.1 Chemicals \& Equipment Required for Synthesis of Fe-Pt Core-Shell Nanoparticles .........69

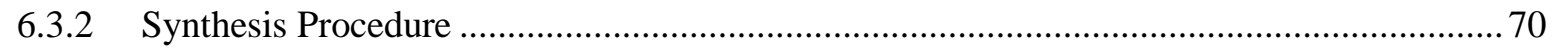

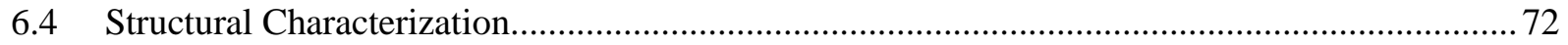

6.4.1 Transmission Electron Microscopy and X-ray Diffraction ............................................... 72

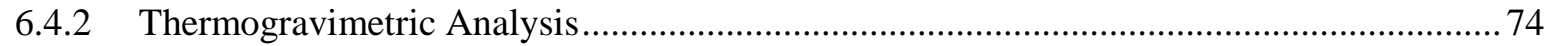

6.5 Temperature and Magnetic Field Dependence of Magnetization .............................................. 75

6.5.1 Zero Field-Cooled and Field-Cooled Magnetization ........................................................ 75

6.5.2 Magnetization as a Function of Applied Magnetic Field Strength...................................... 76

6.6 Temperature Dependence of ac Magnetic Susceptibilities .......................................................... 77

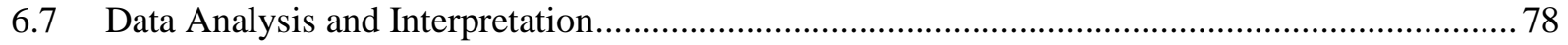

6.7.1 Néel-Brown Relaxation and Interparticle Interactions......................................................78

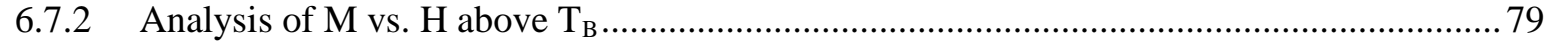

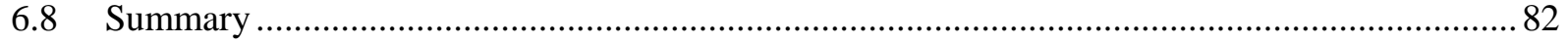

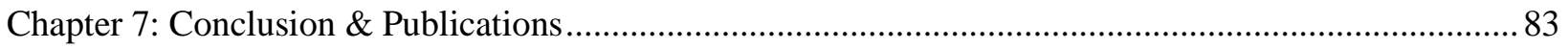

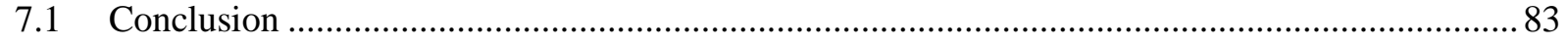

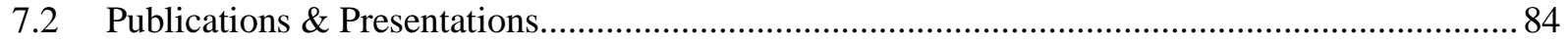

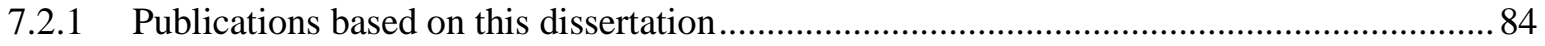

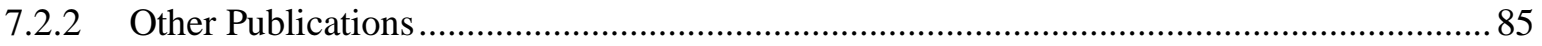

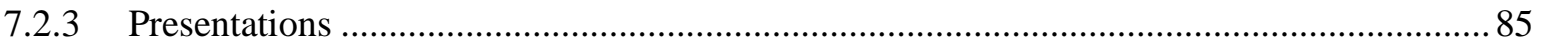

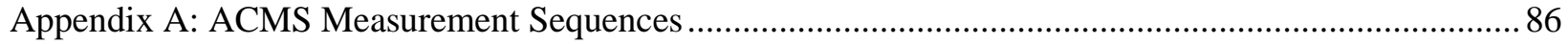

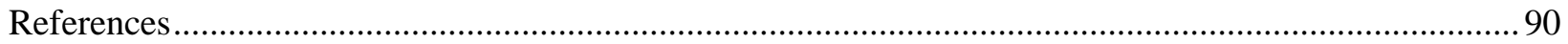




\section{List of Figures}

Fig. $1.1 \quad$ Illustration of spinel structure-page 1

Fig. 1.2 Electron spin configuration in magnetite-page 2

Fig. 2.1 Brillouin function fit to the magnetic moment vs. $\mathrm{H} / \mathrm{T}$ data for $\mathrm{Cr}^{3+}, \mathrm{Fe}^{3+}$, and $\mathrm{Gd}^{3+}$-page 8

Fig. 2.2 Simulated plot of relaxation rate as a function of temperature for $10.3 \mathrm{~nm}$ magnetite-page 11

Fig. 2.3 Simulated relaxation rate as a function of particle diameter at room temperaturepage 12

Fig. 2.4 Simulated relaxation rate as a function of applied magnetic field strength-page 14

Fig. 3.1 Experimental apparatus for nanoparticle synthesis-page 19

Fig. 3.2 Formation of the oleate complex during maghemite synthesis-page 20

Fig. 3.3 Synthesis procedure for maghemite nanoparticles-page 22

Fig. 3.4 Transmission electron micrographs of maghemite nanoparticle samples-page 23

Fig. 3.5 Size distributions of maghemite nanoparticles-page 24

Fig. 3.6 X-ray diffraction pattern for commercial maghemite powder-page 26

Fig. 3.7 X-ray diffraction patterns for maghemite nanoparticle samples-page 26

Fig. 3.8 Williamsen-Hall plots for maghemite nanoparticles-page 27

Fig. 3.9 Thermogravimetric analysis plots of sample weight as a function of temperaturepage 29

Fig. 3.10 Fourier-transform infrared spectra of commercial maghemite powder, pure oleic acid, and $7.0 \mathrm{~nm}$ maghemite nanoparticles coated in oleic acid-page 30

Fig. 3.11 Fourier-transform infrared spectra of maghemite nanoparticle samples with oleic acid coating-page 31 
Fig. 4.1 Apparatus used to prepare magnetic samples-page 34

Fig. 4.2 Sample used for magnetic measurements-page 34

Fig. 4.3 Temperature dependence of the magnetization of $7.0 \mathrm{~nm}$ maghemite nanoparticles under the zero field-cooled and field-cooled conditions-page 36

Fig. 4.4 Hysteresis loop at $2 \mathrm{~K}$ for $7.0 \mathrm{~nm}$ maghemite nanoparticles-page 37

Fig. 4.5 Temperature dependence of the coercivity of $7.0 \mathrm{~nm}$ maghemite nanoparticlespage 38

Fig. 4.6 Magnetic field dependence of the magnetization of $7.0 \mathrm{~nm}$ maghemite nanoparticles at various temperatures above the blocking temperature-page 39

Fig. 4.7 In-phase and out-of-phase ac magnetic susceptibilities of $7.0 \mathrm{~nm}$ maghemite nanoparticles at measuring frequencies ranging from $10 \mathrm{~Hz}$ to $10 \mathrm{kHz}$-page 40

Fig. 4.8 Analysis of the frequency dependence of the blocking temperature of $7.0 \mathrm{~nm}$ maghemite nanoparticles-page 40

Fig. 4.9 Modified Langevin fit to the data of Fig. 4.6-page 42

Fig. 4.10 Curie-Weiss fit to the temperature dependence of the magnetic susceptibility of $7.0 \mathrm{~nm}$ maghemite nanoparticles-page 44

Fig. 4.11 Simulated magnetic moment distributions for different distribution widths-page 46

Fig. 4.12 Difference between the field-cooled and zero field-cooled magnetization fit using a distribution of blocking temperatures-page 49

Fig. 5.1 Zero-field cooled and field-cooled magnetization vs temperature for all maghemite nanoparticle samples-page 52

Fig. 5.2 Hysteresis loops at $2 \mathrm{~K}$ for all maghemite nanoparticle samples-page 53

Fig. 5.3 Temperature dependence of the coercivity and remnant magnetization of the 6.3 nm maghemite nanoparticles-page 53 
Fig. 5.4 Magnetic field dependence of the magnetization for temperatures above the blocking temperature fit to a modified Langevin equation including a distribution of particle magnetic moments for all maghemite nanoparticles-page 54

Fig. 5.5 In-phase and out-of-phase ac susceptibilities of $7.0 \mathrm{~nm}$ maghemite nanoparticles measured at frequencies from $10 \mathrm{~Hz}$ to $5 \mathrm{kHz}$ as a function of temperature-page 55

Fig. 5.6 In-phase and out-of-phase ac susceptibilities of $6.3 \mathrm{~nm}$ maghemite nanoparticles measured at frequencies from $10 \mathrm{~Hz}$ to $5 \mathrm{kHz}$ as a function of temperature-page 56

Fig. 5.7 In-phase and out-of-phase ac susceptibilities of $3.4 \mathrm{~nm}$ maghemite nanoparticles measured at frequencies from $10 \mathrm{~Hz}$ to $5 \mathrm{kHz}$ as a function of temperature-page 56

Fig. $5.8 \quad$ In-phase and out-of-phase ac susceptibilities of $2.5 \mathrm{~nm}$ maghemite nanoparticles measured at frequencies from $10 \mathrm{~Hz}$ to $5 \mathrm{kHz}$ as a function of temperature-page 57

Fig. 5.9 Frequency dependence of the blocking temperatures for all four maghemite nanoparticle samples-page 59

Fig. 5.10 Blocking temperature as a function of particle diameter-page 60

Fig. 5.11 Anisotropy constants as a function of maghemite particle diameter from this dissertation as well as from literature-page 61

Fig. 5.12 Temperature dependent saturation magnetization and particle magnetic moments from the data of Tables 5.3, 5.4, 5.5, and 5.6-page 63

Fig. 5.13 Curie-Weiss fit to the inverse magnetic susceptibility vs temperature-page 66

Fig. 5.14 Interparticle interaction parameter $\Phi$ vs. the ratio of the Vogel-Fulcher temperature to the blocking temperature-page 67

Fig. 6.1 Synthesis procedure for the Fe-Pt core-shell nanoparticles-page 71 
Fig. 6.2 Size distribution of the Fe-Pt nanoparticles including regular and high-resolution transmission electron microscope images-page 72

Fig. 6.3 X-ray diffraction pattern of Fe-Pt nanoparticles with reference patterns for Fe, $\mathrm{Pt}$ and FePt-73

Fig. 6.4 Lattice fringes of a Fe-Pt nanoparticle observed in the transmission electron microscope with a plot of the gray values used to determine the $\mathrm{d}$ spacing-page 73

Fig. 6.5 Thermogravimetric analysis of Fe-Pt nanoparticle sample used to determine the mass of polyvinylpyrrolidone present in the sample-page 74

Fig. 6.6 Temperature dependence of the magnetization of the Fe-Pt nanoparticles for the zero field-cooled and field-cooled cases-page 75

Fig. 6.7 Hysteresis loop for the Fe-Pt nanoparticles at 2 K-page 76

Fig. 6.8 Temperature dependence of the coercivity of Fe-Pt nanoparticles-page 76

Fig 6.9 Magnetic field dependence of the magnetization of Fe-Pt nanoparticles above the blocking temperature-page 77

Fig. 6.10 In-phase and out-of-phase ac magnetic susceptibility of the Fe-Pt nanoparticles measured at frequencies from $10 \mathrm{~Hz}$ to $5 \mathrm{kHz}$-page 78

Fig. 6.11 Vogel-Fulcher law fit to the frequency dependent blocking temperature measurements-page 79

Fig. 6.12 Scaled temperature dependence of the magnetization of Fe-Pt nanoparticles above the blocking temperature-page 79

Fig. 6.13 Data of Fig. 6.9 illustrating the results of the modified Langevin fit for the $50 \mathrm{~K}$ and $100 \mathrm{~K}$ cases ignoring a distribution of particle magnetic moments-page 80

Fig 6.14 Data of Fig. 6.9 illustrating the results of the modified Langevin fit for the $50 \mathrm{~K}$ and $100 \mathrm{~K}$ cases including a distribution of particle magnetic moments-page 81 


\section{List of Tables}

Table 3.1 Synthesis parameters for maghemite nanoparticle formation-page 21

Table 3.2 Size distribution parameters for maghemite nanoparticle samples-page 24

Table 3.3 Results of Williamsen-Hall fit to the X-ray diffraction data of the maghemite nanoparticles-page 27

Table 3.4 Results of thermogravimetric analysis on maghemite nanoparticle samples-page 30

Table 4.1 Magnitudes of the parameters obtained from a fit of the data of Fig. 4.6 to a modified Langevin equation-page 39

Table 4.2 Simulated values of mean magnetic moment, standard deviation, and location of the peak in the lognormal distribution function describing the particle magnetic moments-page 47

Table 4.3 Parameters from a fit of the data of Fig. 4.6 to a modified Langevin fit including a distribution in particle magnetic moments-page 48

Table 5.1 Observed blocking temperatures determined from the peak in the zero fieldcooled data for all four maghemite nanoparticles-page 51

Table 5.2 Vogel-Fulcher temperatures and anisotropy constants for the maghemite nanoparticle samples-page 62

Table 5.3 Parameters for the fit to a modified Langevin ignoring the distribution of particle sizes for the $7.0 \mathrm{~nm}$ maghemite nanoparticles-page 62

Table 5.4 Parameters for the fit to a modified Langevin ignoring the distribution of particle sizes for the $6.3 \mathrm{~nm}$ maghemite nanoparticles-page 62

Table 5.5 Parameters for the fit to a modified Langevin ignoring the distribution of particle sizes for the $3.4 \mathrm{~nm}$ maghemite nanoparticles-page 62 
Table 5.6 Parameters for the fit to a modified Langevin ignoring the distribution of particle sizes for the $2.5 \mathrm{~nm}$ maghemite nanoparticles-page 60

Table 5.7 Calculated estimates for the number of nanoparticles per unit volume for perfectly efficient packing and hexagonal-close-packed assemblies of nanoparticles-page 63

Table 5.8 Parameters for the fit to a modified Langevin equation including a distribution of particle magnetic moments for the $7.0 \mathrm{~nm}$ maghemite nanoparticles-page 64

Table 5.9 Parameters for the fit to a modified Langevin equation including a distribution of particle magnetic moments for the $6.3 \mathrm{~nm}$ maghemite nanoparticles-page 64

Table 5.10 Parameters for the fit to a modified Langevin equation including a distribution of particle magnetic moments for the $3.4 \mathrm{~nm}$ maghemite nanoparticles-page 64

Table 5.11 Parameters for the fit to a modified Langevin equation including a distribution of particle magnetic moments for the $2.5 \mathrm{~nm}$ maghemite nanoparticles-page 65

Table 5.12 Results of the Curie-Weiss fit to the data of Fig. 5.13-page 66

Table 5.13 Summary of key properties of maghemite nanoparticles-page 68

Table 6.1 Parameters from the fit of the magnetic field dependent magnetization of Fe-Pt nanoparticles above the blocking temperature to a modified Langevin equation ignoring the distribution of magnetic moments-page 80

Table 6.2 Parameters from the fit of the magnetic field dependent magnetization of Fe-Pt nanoparticles above the blocking temperature to a modified Langevin equation including a distribution of magnetic moments-page 81 


\section{Chapter 1}

\section{Background \& Motivation}

\subsection{Introduction}

This dissertation deals primarily with the size-dependent magnetic properties of maghemite ( $\gamma$ $\mathrm{Fe}_{2} \mathrm{O}_{3}$ ) nanoparticles with one chapter dedicated to the magnetic properties of iron-platinum core-shell nanoparticles. In this introductory chapter, the properties of bulk magnetite $\left(\mathrm{Fe}_{3} \mathrm{O}_{4}\right)$ and bulk $\gamma-\mathrm{Fe}_{2} \mathrm{O}_{3}$ are introduced and a brief summary of research carried out on $\gamma-\mathrm{Fe}_{2} \mathrm{O}_{3}$ nanoparticles is provided. The motivation for the research presented throughout this dissertation is provided and followed with a brief outline of the work that will be presented in the chapters that follow.

\subsection{Properties of Bulk $\gamma-\mathrm{Fe}_{2} \mathrm{O}_{3}$ and $\mathrm{Fe}_{3} \mathrm{O}_{4}$}

Magnetite $\left(\mathrm{Fe}_{3} \mathrm{O}_{4}\right)$ and maghemite $\left(\gamma-\mathrm{Fe}_{2} \mathrm{O}_{3}\right)$ are the two stable oxides of iron with ferrimagnetic ordering. $\mathrm{Bulk} \mathrm{Fe}_{3} \mathrm{O}_{4}\left(\gamma-\mathrm{Fe}_{2} \mathrm{O}_{3}\right)$ has an inverse spinel structure (Fig. 1.1) with lattice constant $a=0.839$ $\mathrm{nm}(0.835 \mathrm{~nm})$, ferrimagnetic Néel temperature $T_{F N} \cong 948 \mathrm{~K}(600 \mathrm{~K})$, and $8(32 / 3)$ formula units per unit cell. The cations in $\mathrm{Fe}_{3} \mathrm{O}_{4}$ and $\gamma-\mathrm{Fe}_{2} \mathrm{O}_{3}$ are arranged as follows: $8 \mathrm{Fe}_{3} \mathrm{O}_{4}=8\left[\mathrm{Fe}^{3+}\right]_{\mathrm{A}}\left[\mathrm{Fe}^{3+} \mathrm{Fe}^{2+}\right]_{\mathrm{B}} \mathrm{O}_{4}$ and $(32 / 3) \gamma-\mathrm{Fe}_{2} \mathrm{O}_{3}=\left[8 \mathrm{Fe}^{3+}\right]_{\mathrm{A}}\left[(40 / 3) \mathrm{Fe}^{3+}(8 / 3) \mathrm{V}\right]_{\mathrm{B}} \mathrm{O}_{32}$, where $\mathrm{V}$ represents a vacancy. In $\mathrm{Fe}_{3} \mathrm{O}_{4}$ the magnetic

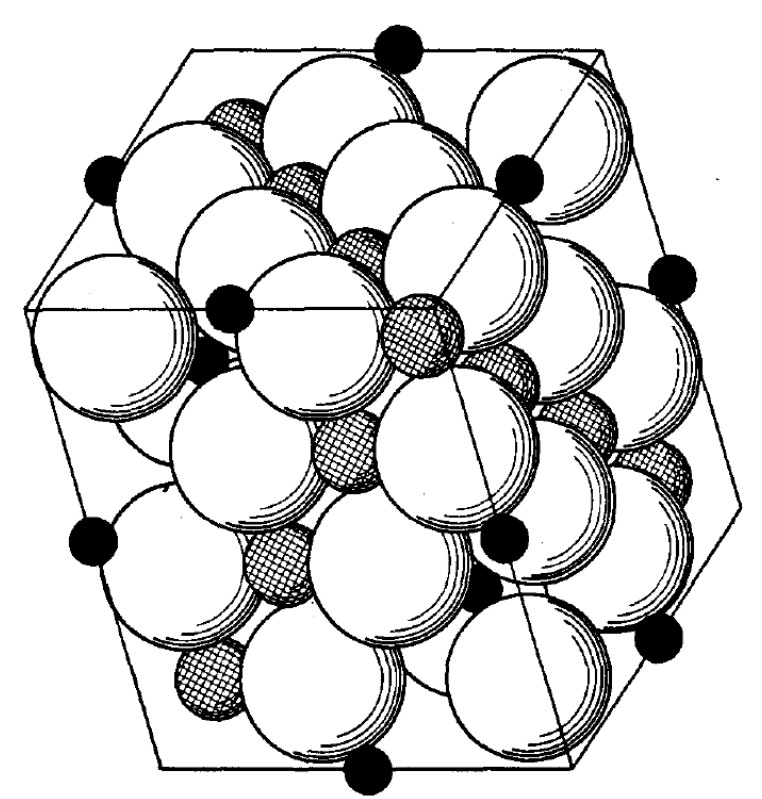

Figure 1.1: Arrangement of atoms in a unit cell with a spinel structure. Large spheres represent the oxygen atoms in the unit cell, cross-hatched spheres represent the octahedral B sites, and the small black spheres represent the tetrahedral A sites.

Reprinted with permission from (Verwey \& Heilmann, 1947). Copyright1947, AIP Publishing LLC. 


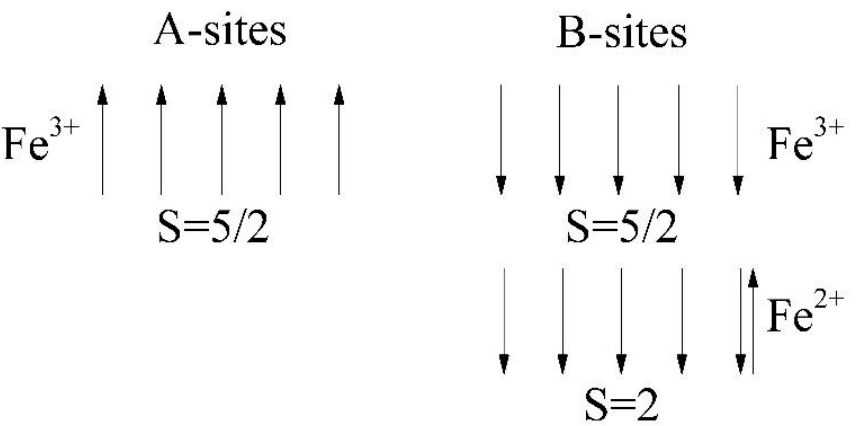

Figure 1.2: Electron configuration of $\mathrm{Fe}_{3} \mathrm{O}_{4}$ formula unit illustrates the canceling of the $\mathrm{Fe}^{3+}$ magnetic moments of the $A$ and $B$ sites.

moments of the $\mathrm{Fe}^{3+}$ ions on the $\mathrm{A}$ sites and the $\mathrm{Fe}^{3+}$ ions on the $\mathrm{B}$ sites cancel each other out so that the net magnetic moment observed is due to the $\mathrm{Fe}^{2+}$ ions only (Fig. 1.2). The saturation magnetization of bulk magnetite can be calculated using a theoretical magnetic moment per $\mathrm{Fe}^{2+}$ ion of $4 \mu_{B}$ yielding the magnetic moment of $32 \mu_{B}$ per unit cell containing 8 formula units. The mass of the unit cell is given by $\rho a^{3}=3.04 \times 10^{-21} \mathrm{~g}$ where $\rho=5.15 \mathrm{~g} / \mathrm{cm}^{3}$ is the density which gives a magnetization value of $98 \mathrm{emu} / \mathrm{g}$ for bulk $\mathrm{Fe}_{3} \mathrm{O}_{4}$. In $\gamma-\mathrm{Fe}_{2} \mathrm{O}_{3}$ there are only $\mathrm{Fe}^{3+}$ ions, but there are $16 / 3$ more $\mathrm{Fe}^{3+}$ ions on $\mathrm{B}$ sites than on the A sites. Each $\mathrm{Fe}^{3+}$ ion has a magnetic moment of $5 \mu_{B}$ so that the magnetic moment per unit cell of $\gamma$ $\mathrm{Fe}_{2} \mathrm{O}_{3}$ is $80 / 3 \mu_{B}$. With $\rho=4.86 \mathrm{~g} / \mathrm{cm}^{3}$, the saturation magnetization of bulk $\gamma-\mathrm{Fe}_{2} \mathrm{O}_{3}$ is $87 \mathrm{emu} / \mathrm{g}$, slightly lower than that of $\mathrm{Fe}_{3} \mathrm{O}_{4}$.

\subsection{Previous Studies on $\gamma-\mathrm{Fe}_{2} \mathrm{O}_{3}$ Nanoparticles}

Magnetic properties of nanoparticles depend not only on the particle size, size distribution, and morphology, but also on factors such as interparticle interactions, interactions between the surface spins and ligands, the presence of defects and degree of ordering of the surface and core spins as well as the interactions between them. To understand these different effects, detailed investigations of the magnetic properties of nanoparticles as a function of size, temperature, magnetic field strength, and measurement frequency are needed. In addition, careful structural characterization is required to understand these results. The novel physics that arises from such investigations as well as the diverse applications of magnetic nanoparticles in catalysis, ferrofluid applications, magnetic storage media, and biomedicine have attracted the attention of researchers worldwide (Thanh, 2012; Gubin, 2009; Fiorani, 2005; Pankhurst, et al., 2003; Pankhurst, et al., 2009; Berry \& Curtis, 2003; Wang, et al., 2012).

Typically, it is assumed that the magnetic moment of a ferrimagnetic particle should align completely with a large applied magnetic field and, as such, the magnetization should saturate. The measured values of the saturation magnetization in experiments are observed to be lower than the bulk 
values. In 1968, Berkowitz et al. attempted to explain this phenomena in micron-sized polycrystalline maghemite in terms of nonmagnetic grain boundaries between nanocrystallites. The model proposed was described by the equation (Berkowitz, et al., 1968)

$$
\frac{M_{S}(D)}{M_{S}(b u l k)}=1-3 g / D
$$

where $M_{S}(D)$ is the saturation magnetization of a nanocrystallite of diameter $D, g$ is the grain boundary thickness, or the thickness of the non-magnetic regions, and $M_{S}(b u l k)$ is the bulk saturation magnetization. This same idea has been applied to nanoparticles of $\gamma-\mathrm{Fe}_{2} \mathrm{O}_{3}$ with the decrease in room temperature saturation magnetization with decreasing particle size often attributed to a core-shell structure consisting of a core of magnetically ordered material and $\sim 1 \mathrm{~nm}$ thick shell of magnetically dead material that does not contribute to the magnetization (Millan, et al., 2007). Mössbauer data has (Pankhurst \& Pollard, 1991) shown that surface spins are not entirely disordered and does not provide evidence for the presence of non-magnetic iron in $\gamma-\mathrm{Fe}_{2} \mathrm{O}_{3}$ nanoparticles (Coey, 1971). Coey argued that the reduced magnetization observed in $\gamma-\mathrm{Fe}_{2} \mathrm{O}_{3}$ nanoparticles was due to canting of spins at the surface of particles caused by missing nearest neighbors. This model of noncolinear surface spins in $\gamma-\mathrm{Fe}_{2} \mathrm{O}_{3}$ nanoparticles has been explained in terms of unbalanced superexchange between cations near the surface by Morrish and Haneda who estimated that, in their sample of $6.5 \mathrm{~nm}$ particles, the canting angle was 25-27 degrees showing little dependence on the applied field strength (Morrish \& Haneda, 1983). These effects become increasingly important for nanoparticles where a significant portion of the magnetic ions are located at or near the surface.

Surface spin canting is not the only contributor to the reduced magnetization of $\gamma-\mathrm{Fe}_{2} \mathrm{O}_{3}$ nanoparticles. In addition to surface spin canting, the presence of disorder in the core spins has also been inferred from observation of large coercivity and large high-field susceptibilities in $\gamma-\mathrm{Fe}_{2} \mathrm{O}_{3}$ nanoparticles (Parker, et al., 1993; Morales, et al., 1999). The cationic vacancies in $\gamma-\mathrm{Fe}_{2} \mathrm{O}_{3}$ may be ordered and evenly distributed such that there is minimal core spin canting; however, as the degree of vacancy ordering decreases, inhomogeneities in the core of the nanoparticles can lead to significant reduction in the magnetization (Grau-Crespo, et al., 2010). Furthermore, the crystallinity of the nanoparticles has been shown to effect the magnetic behavior significantly. Serna et al. reported coercivity of 700 Oe and 3000 Oe for two different $3.5 \mathrm{~nm}$ samples prepared by the same technique at different temperatures (Serna, et al., 2001). Thus, the synthesis procedure may also affect the magnetic properties significantly.

Studies on several different size nanoparticles of $\gamma-\mathrm{Fe}_{2} \mathrm{O}_{3}$ dispersed in a polystyrene resin matrix have addressed the issue of the size distribution on the measured dc magnetic properties (Vaishnava, et al., 2007) although, without ac measurements, no information can be gained about effects of interparticle 
interactions nor can a determination of the relaxation rate be carried out (Singh, et al., 2009; Seehra, et al., 2010). In previous studies of $\gamma-\mathrm{Fe}_{2} \mathrm{O}_{3}$ nanoparticles, ac measurements have not been carried out (Dutta, et al., 2004; Vaishnava, et al., 2007). Fiorani et al. did do ac measurements on particles of different sizes, but made no investigation into the effects of the particle size distributions (Fiorani, et al., 2002). Others, such as Nadeem et al., accounted for size distribution but looked only at a single sample (Nadeem, et al., 2011).

\subsection{Motivation for this Research}

Because $\mathrm{Fe}_{3} \mathrm{O}_{4}$ and $\gamma-\mathrm{Fe}_{2} \mathrm{O}_{3}$ have high values of $T_{F N}$, the measured magnetization of each is expected to be only slightly lower than the saturation magnetization values calculated in section 1.2 even at room temperature. These high magnetization values coupled with the resistance to oxidation of $\mathrm{Fe}_{3} \mathrm{O}_{4}$ and $\gamma-\mathrm{Fe}_{2} \mathrm{O}_{3}$ as compared with pure iron and their high biocompatibility when compared with other magnetic materials make $\mathrm{Fe}_{3} \mathrm{O}_{4}$ and $\gamma-\mathrm{Fe}_{2} \mathrm{O}_{3}$ highly suitable for biomedical applications (Thanh, 2012; Pankhurst, et al., 2003; Pankhurst, et al., 2009; Berry \& Curtis, 2003; Wang, et al., 2012). Realizing these applications requires detailed understanding not only of the interactions of nanomaterials with biological environments, but also of the details of the magnetic behavior of the nanoparticles.

To date, much of the published work presented has either ignored any distribution in particle size or the effects of interparticle interactions. In this work, detailed ac and dc magnetic characterization is presented and discussed in terms of a distribution of particle sizes and magnetic moments. The ac magnetic data is used to determine the contribution of interparticle interactions to the observed blocking temperature for different sized nanoparticles. The anisotropy constants for the nanoparticles are determined and the results are considered alongside the work of others who have attempted to account for interparticle interactions. The anisotropy shows a trend that is markedly different than the $1 / D$ behavior typically used to discuss nanoparticle anisotropy. In addition, the validity of the approach across multiple types of magnetic nanoparticles will be demonstrated by utilizing the same investigation techniques for the analysis of very small iron-platinum core-shell magnetic nanoparticles.

\subsection{Dissertation Outline}

This dissertation consists of 7 chapters (including this one). The general outline for the rest of the dissertation is as follows. Chapter 2 deals with the theoretical framework necessary for the discussion of magnetic behavior. For clarity, much of the information in Chapter 2 is presented for a single spin or single magnetic moment rather than an assembly of particles or moments with a distribution of sizes or magnitudes. After the background material has been covered, details of the synthesis and structural characterization of oleic acid-coated $\gamma-\mathrm{Fe}_{2} \mathrm{O}_{3}$ nanoparticles are described in Chapter 3. The techniques 
used are described and the results dealing with the magnetic behavior are presented. Chapter 4 covers the procedure for magnetic measurements presented in this dissertation and the magnetic properties of the 7.0 $\mathrm{nm} \gamma-\mathrm{Fe}_{2} \mathrm{O}_{3}$ nanoparticles in detail. The majority of the results presented in Chapter 4 have been published in a recent paper (Pisane, et al., $2015 \mathrm{I}$ ). After the detailed discussion of the $7.0 \mathrm{~nm}$ particles, Chapter 5 will focus on the size dependent magnetic properties of $\gamma-\mathrm{Fe}_{2} \mathrm{O}_{3}$ nanoparticles. The same methods of Chapter 4 are applied to nanoparticles of $2.5 \mathrm{~nm}, 3.4 \mathrm{~nm}$, and $6.3 \mathrm{~nm}$ and the results are discussed as a function of particle size. Chapter 6 will briefly discuss the results of applying the characterization techniques of Chapters $3 \& 4$ to iron-platinum core-shell magnetic nanoparticles (Pisane, et al., 2015 II). Finally, Chapter 7 will provide a summary of the key results of the work presented in Chapters 3-6. 


\section{Chapter 2}

\section{Theoretical Background}

\subsection{Introduction}

In this chapter, some of the basic equations used to interpret the data presented in this dissertation are described. Unless otherwise indicated, the background information given here is at the level of material given in the text books by Morrish (1955) and Kittel (1976). The purpose of this chapter is to provide the necessary framework to discuss the results of the experiments in the chapters that follow.

\subsection{Temperature \& Magnetic Field Dependence of Magnetization}

The magnetic moment $\vec{\mu}$ is related to the angular momentum $\vec{J}$ by the equation

$$
\vec{\mu}=-\frac{g \mu_{B}}{\hbar} \vec{J}
$$

where $\mu_{B}$ is the Bohr magneton, $\hbar$ is Plank's constant, $\vec{J}$ is the total angular momentum operator, and $g$ is a dimensionless proportionality constant called the $g$ factor. For a non-interacting electron,

$$
\vec{J}=\vec{S}+\vec{L}
$$

where $\vec{S}$ is the spin angular momentum, $\vec{L}$ is the orbital angular momentum, and

$$
g=1+\frac{J(J+1)+S(S+1)-L(L+1)}{2 J(J+1)}
$$

is the Landé g-factor. From Eq. 2.2 and 2.3 it is seen that if $L=0$, then $J=S$ and $g=2$. Alternatively, if $S=0$, then $J=L$ and $g=1$. Given the magnetic moment, the energy of the system in a magnetic field is calculated next. The Hamiltonian for a magnetic moment in a magnetic field is given by

$$
\mathcal{H}=-\vec{\mu} \cdot \vec{H}
$$

where $\vec{H}$ is the magnetic field. Combining equations 2.1 and 2.4 to determine the energy levels gives

$$
E_{j m}=\frac{g \mu_{B}}{\hbar}\langle j m|\vec{H} \cdot \vec{J}| j m\rangle
$$

For $\vec{H}=H \hat{z}$, Eq. 2.5 simplifies to

$$
E_{m}=\frac{g \mu_{B}}{\hbar} H\left\langle j m\left|J_{z}\right| j m\right\rangle=g \mu_{B} H m
$$

where $m$ is the magnetic or spin quantum number which describes the orientation of an orbital in space and can have any integer value from $-J$ to $J$. For $H=0$ Oe, these energy levels are degenerate; however, 
an applied magnetic field splits the energy levels so that the difference between each state is proportional to the field strength $H$, a phenomenon known as Zeeman splitting. According to statistical mechanics, the free energy is given by

$$
F=-k_{B} T \ln \sum_{m} \exp \left[-E_{m} / k_{B} T\right]
$$

where $k_{B}$ is the Boltzmann constant and $T$ is the temperature. The magnetization $M$ defined as magnetic moment per unit volume is given by

$$
M=-\frac{1}{V} \frac{\partial F}{\partial H}
$$

where $V$ is the particle volume. Substituting Eq. 2.7 into Eq. 2.8 gives the van Vleck equation:

$$
M=-\frac{1}{V} \frac{\sum_{-J}^{J} g \mu_{B} m \exp \left[-g \mu_{B} H m / k_{B} T\right]}{\sum_{-J}^{J} \exp \left[-g \mu_{B} H m / k_{B} T\right]} .
$$

Performing the sum in Eq. 2.9 gives

$$
M=N g \mu_{B} B_{J}(x)
$$

where

$$
B_{J}(x)=\frac{2 J+1}{2 J} \operatorname{coth}\left(\frac{2 J+1}{2 J} x\right)-\frac{1}{2 J} \operatorname{coth}\left(\frac{1}{2 J} x\right)
$$

is the Brillouin function where $x=g \mu_{B} H / k_{B} T$ and $N=1 / V$ is the number of magnetic atoms/ions per unit volume. In the low-field, high temperature limit $x \ll 1$ and, using $\operatorname{coth}(y) \cong y^{-1}+\frac{1}{3} y+\cdots$ for $y \ll 1$, Eq. 2.10 reduces to

$$
M=\frac{N g^{2} J(J+1) \mu_{B}^{2} H}{3 k_{B} T}=\frac{N \mu^{2} H}{3 k_{B} T}=\frac{C H}{T}
$$

Eq. 2.12 is the Curie Law. Alternatively, in the high-field, low temperature limit where $H / T$ is large, Eq. 2.10 begins to saturate as $B_{J}(x) \rightarrow 1$ for $x \rightarrow \infty$. These results can be seen from the plot of the Brillouin function shown in Fig. 2.1. Note that here $\mu^{2}=g^{2} J(J+1) \mu_{B}{ }^{2}$ is the magnetic moment per atom (ion). If the magnetization follows the inverse temperature dependence of the Curie Law given by Eq. 2.12, then the Curie constant $C=N \mu^{2} / 3 k_{B}$ can be determined which, in turn, yields the magnetic moment $\mu$ of the system. In the low temperature, high-field limit when $B_{J}(x) \rightarrow 1$, Eq. 2.10 reduces to $M=N g \mu_{B} J$. Thus, the saturation magnetization can be used to determine the quantity $g \mu_{B} J$. 


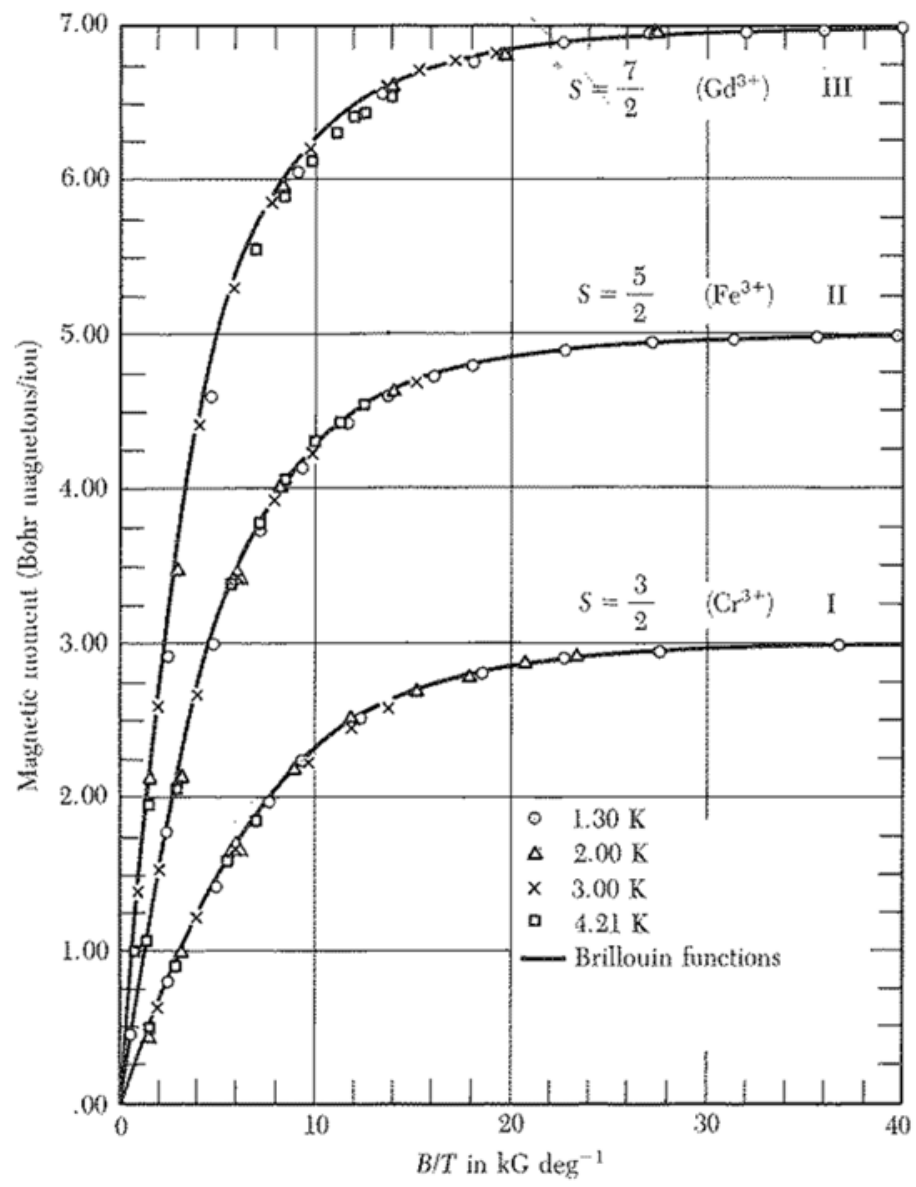

Figure 2.1: Fit of the magnetic moment data for $\mathrm{Cr}^{3+}(S=3 / 2), \mathrm{Fe}^{3+}(S=5 / 2)$, and $\mathrm{Gd}^{3+}(S=7 / 2)$, as a function of $\mathrm{H} / \mathrm{T}$ to the Brillouin function, Eq. 2.10.

Reprinted with permission from Kittel (1976). Copyright 1976, John Wiley \& Sons, Inc.

For the case where $J \rightarrow \infty$, the classical case when the moments can orient in all directions, $(2 J+$ $1) / 2 J \rightarrow 1$. Also, $(1 / 2 J) \operatorname{coth}(x / 2 J)=\frac{1}{x}\left(\frac{x}{2 J}\right) \operatorname{coth}\left(\frac{x}{2 J}\right) \rightarrow 1 / x$ since $y \times \operatorname{coth} y \rightarrow 1$ as $y \rightarrow 0$. In this case,

$$
B(x)=\operatorname{coth}(x)-\frac{1}{x}=L(x)
$$

where $L(x)$ is called the Langevin function. This equation will be used in the case of superparamagnets for which the magnetic moment per particle can be $\sim 10^{3} \mu_{B}$ or larger.

\subsection{Curie-Weiss Law}

Until now, no efforts have been made to account for interactions between ions; however, in a solid the magnetic moments of the individual atoms are able to interact with one another. In 1905, Weiss 
proposed that the effects on a given ion due to the magnetic moments of the other ions in a solid be replaced by a molecular field

$$
\overrightarrow{H_{m}}=\gamma \vec{M}
$$

where $\gamma$ is a constant and $\vec{M}$ is the magnetization. Replacing $H$ in Eq. 2.12 with $H_{o}+H_{m}$ yields

$$
M=\frac{C}{T}\left(H_{o}+\gamma M\right)
$$

which, when solved, gives

$$
M=\frac{C H_{o}}{T-\theta} \text { or } \chi=\frac{C}{T-\theta}
$$

where $\theta=\gamma C$. Eq. 2.16 is the Curie-Weiss law. According to the Curie-Weiss law, when $T \rightarrow \theta, \chi \rightarrow \infty$ so that there is a magnetization even in the absence of an applied field, such as in the case of ferromagnetic materials. Additional background on the molecular field theory presented in this section can be found in Smart (1966).

\subsection{Exchange Interaction}

In Section 2.3, the effect of interactions between an ion in a crystal and the rest of the crystal was discussed without any explanation of the source of this interaction. If one were to calculate the molecular field in a permanent magnet, it would be too large to be explained by dipole-dipole interactions. The extra forces required to align the magnetic moments of the atoms in magnetic materials are due to exchange interactions. Exchange interactions are quantum mechanical and the value of $\gamma$ in Eq. 2.14 can only be determined using quantum mechanics.

If one considers two electrons at points $a$ and $b$ with spin angular momentum $\overrightarrow{S_{a}}$ and $\overrightarrow{S_{b}}$, respectively, their exchange energy can be described by

$$
\mathcal{H}_{e x}=-2 J_{a b} \overrightarrow{S_{a}} \cdot \overrightarrow{S_{b}}
$$

where $J_{a b}$ is the exchange integral for the interaction between ions $a$ and $b$ which is positive for ferromagnetic materials (so that the lowest energy state is one where the spins are parallel) and negative for antiferromagnetic materials (so that the lowest energy state is one where the spins are antiparallel). The exchange integral for electrons 1 and 2 at sites $a$ and $b$ is given by

$$
J_{a b}=\int \psi_{a}^{*}\left(r_{1}\right) \psi_{b}^{*}\left(r_{2}\right) \frac{e^{2}}{r_{12}} \psi_{b}\left(r_{1}\right) \psi_{a}\left(r_{2}\right) d^{3} r_{1} d^{3} r_{2}
$$


and depends on the overlap of the wave functions of the electrons, $\psi_{a}\left(r_{1}\right)$ and $\psi_{b}\left(r_{2}\right)$, and the Pauli repulsion. If there are $z$ nearest neighbors with which the exchange is significant, then the exchange energy is a sum over all of the nearest neighbors $(\mathrm{nn})$ :

$$
\begin{aligned}
& \mathcal{H}_{\text {ex }}=-2 \sum_{a \neq b}^{n n} J_{a b} \overrightarrow{S_{a}} \cdot \overrightarrow{S_{b}} \\
& \left\langle\mathcal{H}_{\text {ex }}\right\rangle=-2 J_{a b}\left\langle\overrightarrow{S_{a}}\right\rangle \cdot \sum_{b=1}^{z}\left\langle\overrightarrow{S_{b}}\right\rangle .
\end{aligned}
$$

Let $J_{a b}=J_{e}$ be the nearest neighbor exchange constant, and equating Eq. 2.20 with Eq. 2.4 gives:

$$
\begin{gathered}
\left\langle\mathcal{H}_{e x}\right\rangle=-g \mu_{B} H_{m} \cdot\left\langle\overrightarrow{S_{b}}\right\rangle \\
2 J_{e} Z\left\langle S_{b}\right\rangle=g \mu_{B} H_{m} \\
H_{m}=\frac{2 J_{e} Z\left\langle S_{b}\right\rangle}{g \mu_{B}}
\end{gathered}
$$

and one can see that $\gamma \propto J_{e}$. Then, substituting for $M$ using Eq. 2.12 and solving for $\gamma$ gives

$$
\gamma=\frac{2 J_{e} z}{N g^{2} \mu_{B}^{2}}
$$

From here, one finds

$$
\theta=\frac{2 z J_{e} S(S+1)}{3 k_{B}}
$$

which is the Curie temperature often denoted as $T_{C}$. Below $T_{C}$, the spins will align parallel or antiparallel (ferromagnetic or antiferromagnetic coupling) depending on the sign of $J_{e}$ while above $T_{C}$ they will behave as paramagnets. The information presented in this section can be found in more detail in Kittel (1976).

\subsection{Superparamagnetism}

In section 2.3, the Curie-Weiss law for a single spin was described. Now, a small maghemite particle of volume $V$ below its ferrimagnetic ordering temperature $T_{F N} \sim 600 \mathrm{~K}$ is considered. In the absence of an applied field, the height of the energy barrier between the parallel and antiparallel directions of magnetization is given by

$$
\Delta E=K_{a} V
$$

where $K_{a}$ is the anisotropy constant. From Eq. 2.26, it is seen that as the particle size decreases, the energy barrier is also decreased. For small enough particles this barrier height becomes comparable with the thermal energy: 


$$
K_{a} V \sim k_{B} T
$$

where $\mathrm{k}_{\mathrm{B}}$ is the Boltzman constant and $T$ is the temperature. When this happens, the particle has enough thermal energy for its moment to switch between the parallel and antiparallel directions and so it behaves like a paramagnet. There is a critical size below which the entire magnetic moment of the particle behaves like a single spin except with a very large magnetic moment; hence the term superparamagnetism. This size can be estimated from Eq. 2.27: at room temperature ( $300 \mathrm{~K})$, the thermal energy is $4.14 \times 10^{-14} \mathrm{erg}$; using $K_{a}=4.7 \times 10^{4} \mathrm{erg} / \mathrm{cm}^{3}$ for bulk magnetite (Fiorani, et al., 2002), a critical $V \approx 8.81 \times 10^{-19} \mathrm{~cm}^{3}$ is found which, for a spherical particle, corresponds to a diameter $D \approx 11.9 \mathrm{~nm}$. Therefore, superparamagnetism is only observed in very small particles.

\subsubsection{Magnetic Relaxation and Blocking Temperature}

Now, a nanoparticle with uniaxial anisotropy along the z-axis is considered; such a system has two equivalent directions of magnetization: $+\hat{z}$ and $-\hat{z}$ where the energy barrier to switching, $\Delta E$, between these equivalent magnetization directions is a function of the angle between the $\mathrm{z}$-axis and the magnetization. A superparamagnetic particle that is in a field will have its magnetic moment aligned with the field. When the applied field is turned off, the magnetization will begin to decay as the field is no longer able to force the magnetic moments to align in any particular direction. To characterize this decay, the magnetization is first described as a function of time after the field is switched off by

$$
M=M(0) \exp (-t / \tau)
$$

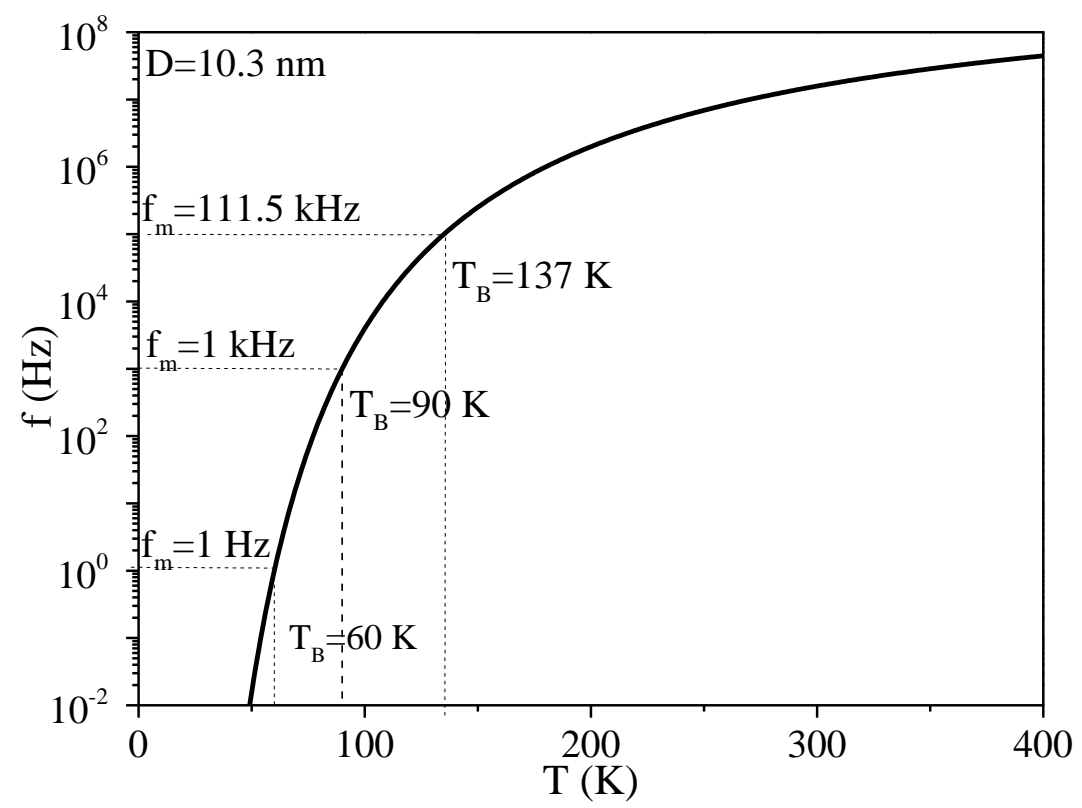

Figure 2.2: Solid curve is relaxation rate vs. Temperature for a $10.3 \mathrm{~nm}$ magnetite nanoparticle. Dashed lines indicate the blocking temperature that would be observed for select frequencies. 


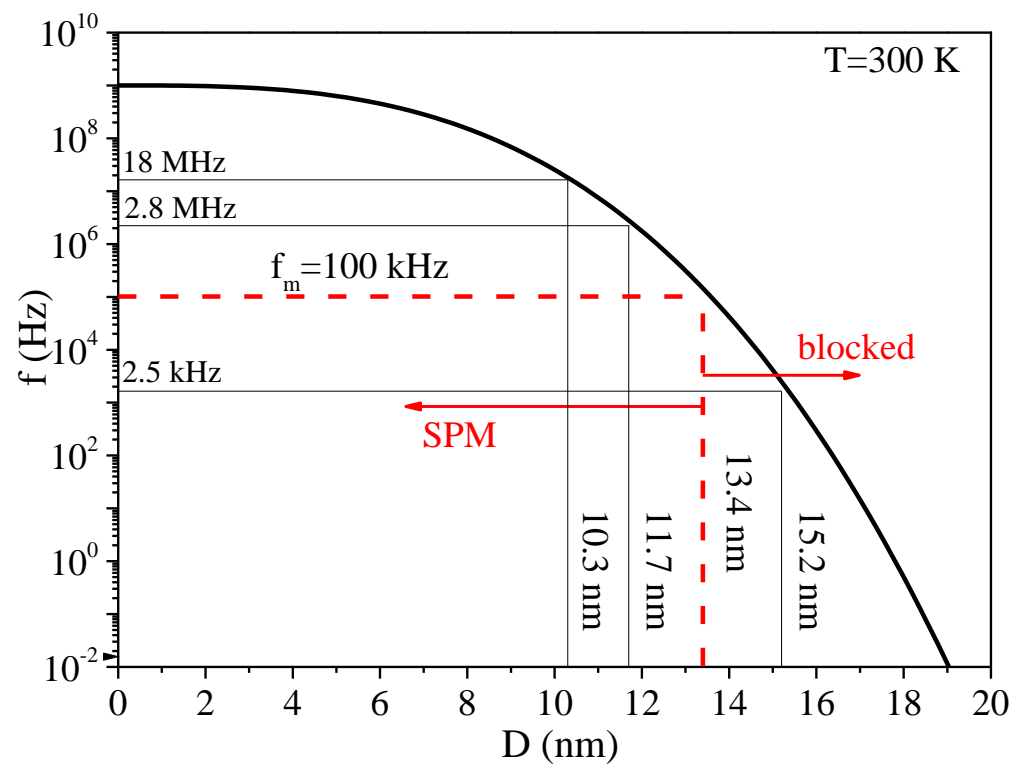

Figure 2.3: Size dependence of the relaxation rate for nanoparticles with $K_{a}=3 \times 10^{5} \mathrm{erg} / \mathrm{cm}^{3}$ at $T=300 \mathrm{~K}$. Lines drawn are to show the different relaxations rates for particles of different diameters $(10.3 \mathrm{~nm}, 11.7 \mathrm{~nm}, 13.4 \mathrm{~nm}$, and $15.2 \mathrm{~nm})$ at $300 \mathrm{~K}$.

where $M(0)$ is the magnetization at the instant the field is turned off, $t$ is the time in seconds after the field has been turned off, and $\tau=1 / f$ is the relaxation time. From statistical mechanics, the probability of overcoming an energy barrier $\Delta E$ due to thermal energy $k_{B} T$ is given by

$$
\exp \left[-\Delta E / k_{B} T\right]
$$

and so the relaxation rate can be written as

$$
f=1 / \tau=f_{o} \exp \left[-\frac{\Delta E}{k_{B} T}\right]
$$

where $f_{o}$ is the attempt frequency. Eq. 2.30 is known as the Néel relaxation equation (Néel, 1949; Néel, 1955). Plugging Eq. 2.26 into 2.30, it becomes clear that $f$ will increase with increasing temperature (Fig. 2.2) and decrease with increasing particle size (Fig 2.3).

If the relaxation rate is high enough that the magnetic moments can switch direction during a measurement, the measured magnetic moment will average out to zero. Because the relaxation rate in Eq. 2.30 depends on temperature, the temperature for which $f=f_{m}$ is defined as the blocking temperature and given by

$$
T_{B}=T_{a} / \ln \left(f_{o} / f_{m}\right) \text { with } T_{a}=K_{a} V / k_{B}
$$

where $f_{m}$ is the inverse of the time it takes to complete a single measurement. This $T_{B}$ is the temperature, below which, a net magnetization is observed for the particles and they are said to be 
'blocked' from switching direction of magnetization and, above which, the measured magnetization is zero due to the switching of magnetization direction. The dashed lines in Fig 2.2 represent blocking temperatures observed for a $10.3 \mathrm{~nm}$ magnetic nanoparticle at select measurement frequencies.

\subsubsection{Effects of Magnetic Field on the Energy Barrier}

Magnetic anisotropy is the directional dependence of the magnetic properties of a material. If the nanoparticle has one easy axis of magnetization, then the anisotropy energy for a particle with uniaxial anisotropy is given by

$$
E_{k}=K_{a} V \sin ^{2} \theta
$$

where $\theta$ is the angle between the magnetic moment and the anisotropy axis, $V$ is the particle volume, and $K_{a}$ is the anisotropy constant. When applying a field along the easy axis of magnetization so that $\theta$ is the angle between the magnetic moment and the applied field direction, the total energy of the system becomes

$$
E=K_{a} V \sin ^{2} \theta-\mu H \cos \theta
$$

To determine the effective barrier height in an applied magnetic field, the minimum and maximum values of $E$ must be determined. Taking

$$
\frac{\partial E}{\partial \theta}=2 K_{a} V \sin \theta \cos \theta+\mu H \sin \theta=0
$$

yields $\theta=0, \pi, \cos ^{-1}\left(-\mu H /\left(2 K_{a} V\right)\right)$. Evaluating $E$ at each of these values of $\theta$ gives two local minima $( \pm \mu H)$ and a maximum $K_{a} V\left(1+\left(\frac{\mu H}{2 K_{a} V}\right)^{2}\right)$. The energy barrier to switching from the antiparallel to parallel state is then

$$
\Delta E=E_{\text {max }}-E_{\text {antiparallel }}=K_{a} V\left(1+\left(\frac{\mu H}{2 K_{a} V}\right)^{2}\right)-\mu H=K_{a} V\left(1-\frac{\mu H}{2 K_{a} V}\right)^{2} .
$$

Eq. 2.35 describes the energy barrier that a magnetic moment must overcome in an applied magnetic field in order to align with the field. From the negative sign in Eq. 2.35, it is clear that an applied field lowers the barrier to switching and that, for $H=2 K_{a} V / \mu$, the barrier disappears. This value, often denoted $H_{k}$, represents the anisotropy field for a magnetic moment, this is the magnetic field strength required to force the moment to align with the magnetic field direction. The relaxation rate for the $10.3 \mathrm{~nm} \gamma-\mathrm{Fe}_{2} \mathrm{O}_{3}$ nanoparticle of Fig 2.2 is plotted as a function of applied magnetic field strength in Fig. 2.4. 


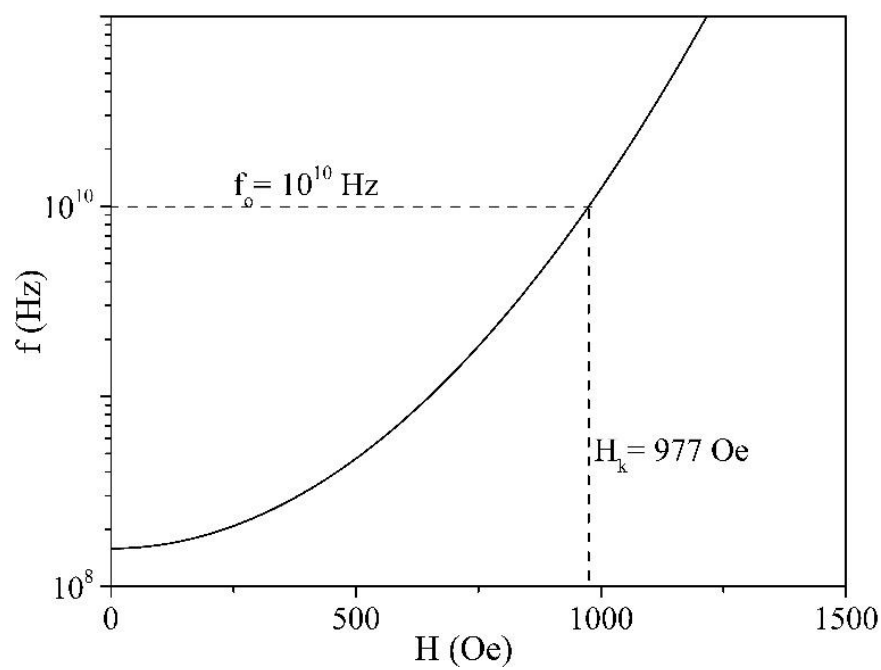

Figure 2.4: Relaxation rate vs. applied magnetic field for $T=300 \mathrm{~K}, K_{a}=3 \times 10^{5} \mathrm{erg} / \mathrm{cm}^{3}, D=10.3$ $n m$. The rate of relaxation increases with increasing $H$.

\subsection{Magnetic Field Dependence of Magnetization at Temperatures above $T_{B}$}

In a superparamagnetic nanoparticle, a large number of spins can contribute collectively to the magnetic moment making $\mu \gg \mu_{B}$. Since the spins are coupled, the net result is a very large effective spin such that $J$ is very large and $m$ may take on many different values. The sum in Eq. 2.9 then becomes an integral and the Brillouin function can be simplified to the Langevin function;

$$
B(x) \rightarrow L(x)=\operatorname{coth}(x)-1 / x
$$

so that

$$
M=N \mu L(x)=M_{o} L\left(\frac{\mu_{p} H}{k_{B} T}\right)
$$

where $\mu_{p}$ is the magnetic moment per particle and $M_{o}$ is the temperature dependent saturation magnetization. In practice, canted spins at the surface of nanoparticles may contribute a high-field magnetic susceptibility $\left(\chi_{a}\right)$, and an additional term is added to Eq. 2.37 (Makhlouf, et al., 1997; Punnoose, et al., 2004):

$$
M=M_{o} L\left(\frac{\mu_{p} H}{k_{B} T}\right)+\chi_{a} H
$$

Eq. 2.38 and slight modifications of it will be used throughout this dissertation to analyze the magnetic response of nanoparticles at temperatures above $T_{B}$. 


\subsection{Effects of Size Distribution}

In a sample with more than one nanoparticle, there will be some variation in particle size and the diameters of the particles $(D)$ can typically be described by a lognormal distribution (Richardson \& Desai, 1976):

$$
f(D)=\frac{1}{D \lambda_{D} \sqrt{2 \pi}} \exp \left\{-\frac{\left[\ln \left(D / D_{O}\right)\right]^{2}}{2 \lambda_{D}{ }^{2}}\right\}
$$

where $D_{o}$ is the median value of $D$ and $\lambda_{D}$ describes the width of the distribution. The average diameter can be found by integrating the distribution function:

$$
\begin{gathered}
\langle D\rangle=\int_{0}^{\infty} D f(D) d D=\frac{1}{\lambda_{D} \sqrt{2 \pi}} \int_{0}^{\infty} \exp \left\{\frac{-\left[\ln \left(D / D_{o}\right)\right]}{2 \lambda_{D}{ }^{2}}\right\} d D \\
=\lim _{D \rightarrow \infty} \frac{D_{o}}{2} \exp \left(\frac{\lambda_{D}{ }^{2}}{2}\right) \operatorname{erf}\left[\frac{\ln \left(D / D_{o}\right)-\lambda_{D}{ }^{2}}{\sqrt{2} \lambda_{D}}\right]-\lim _{D \rightarrow 0} \frac{D_{o}}{2} \exp \left(\frac{\lambda_{D}{ }^{2}}{2}\right) \operatorname{erf}\left[\frac{\ln \left(D / D_{o}\right)-\lambda_{D}{ }^{2}}{\sqrt{2} \lambda_{D}}\right] \\
=D_{o} \exp \left[\frac{\lambda_{D}{ }^{2}}{2}\right] .
\end{gathered}
$$

The parameter $\lambda_{D}$ is related to the mean and standard deviation of the distribution by

$$
\lambda_{D}=\sqrt{\ln \left(1+\frac{\sigma_{D}^{2}}{\langle D\rangle^{2}}\right)}
$$

so that the standard deviation is given by

$$
\sigma_{D}=\langle D\rangle\left[\exp \left(\lambda_{D}^{2}\right)-1\right]^{1 / 2}
$$

Because the energy barrier given in Eq. 2.26 depends on particle volume, the effects of a distribution of particle sizes on the observed magnetic data can be significant as will be shown in Chapter 4.

\subsection{Temperature and Frequency Dependence of ac Susceptibilities}

In Chapters $4 \& 5$, data on the temperature and frequency dependence of the ac susceptibilities, $\chi^{\prime}$ and $\chi^{\prime \prime}$, in various samples of maghemite will be presented. Here basic equations governing $\chi^{\prime}$ and $\chi^{\prime \prime}$ are derived. For ac measurements, the magnetic field varies with time and

$$
\vec{M}=\chi \vec{H}
$$

can be replaced with 


$$
\vec{M}=\chi \overrightarrow{H_{o}} \exp [i \omega t]
$$

where $H_{o}$ is the ac field amplitude, $\omega=2 \pi f_{m}$ where $f_{m}$ is the frequency of the ac magnetic field (measuring frequency) in $\mathrm{Hz}, t$ is the time in seconds, and $i=\sqrt{-1}$. The ac magnetic susceptibility $\chi$ can be determined by approximating the rate of change of the magnetization with

$$
\frac{d \vec{M}}{d t}=f\left[\overrightarrow{M_{o}}-\vec{M}\right]
$$

where

$$
\overrightarrow{M_{o}}=\chi_{o} \vec{H}
$$

is the equilibrium magnetization with $\chi_{o}$ as the dc initial susceptibility and $f$ is the relaxation rate given by

$$
f=1 / \tau=f_{o} \exp \left[-\frac{\mathrm{K}_{a} V}{k_{B} T}\right]
$$

Here, Eq. 2.47 is a restatement of Eq. 2.30 with $\Delta E=K_{a} V$. First, Eq. 2.46 and Eq. 2.47 are substituted into Eq. 2.45 to yield

$$
\frac{d \vec{M}}{d t}=f \overrightarrow{H_{o}} \exp [i \omega t]\left[\chi_{o}-\chi\right]
$$

Differentiating Eq. 2.44, substituting the result into the left side of Eq. 2.48, and simplifying gives

$$
\chi=\chi^{\prime}-i \chi^{\prime \prime}=\frac{\chi_{o}}{1+i \omega \tau}
$$

where $\tau=1 / f, \chi^{\prime}=M^{\prime} / H_{o}$ is the in-phase ac magnetic susceptibility, and $\chi^{\prime \prime}=M^{\prime \prime} / H_{o}$ is the out-ofphase magnetic susceptibility. Then, the components can be expressed as (Andersson, et al., 1997; Lundgren, et al., 1981):

$$
\chi^{\prime}=\frac{\chi_{o}}{1+(\omega \tau)^{2}}
$$

and

$$
\chi^{\prime \prime}=\frac{\chi_{o} \omega \tau}{1+(\omega \tau)^{2}}
$$

For randomly oriented nanoparticles each with magnetic moment $\mu=M_{S} V$, Eq. 2.50 and Eq. 2.51 can be written as (Andersson, et al., 1997):

$$
\chi^{\prime}=\frac{M_{S}}{3 K_{a}}\left\{1+\frac{K_{a} V}{k_{B} T} \frac{1}{1+(\omega \tau)^{2}}\right\}
$$


and

$$
\chi^{\prime \prime}=\frac{M_{S}}{3 K_{a}}\left\{\frac{K_{a} V}{k_{B} T} \frac{\omega \tau}{1+(\omega \tau)^{2}}\right\}
$$

It can be shown from Eq. 2.52 and Eq. 2.53 that $\chi^{\prime}$ and $\chi^{\prime \prime}$ are related by (Lundgren, et al., 1981)

$$
\chi^{\prime \prime}=C \frac{\partial\left[T \chi^{\prime}\right]}{\partial T}
$$

where

$$
C \cong \frac{\pi}{2 \ln \left[f_{o} / 2 \pi f_{m}\right]}
$$

and that $\chi^{\prime \prime}$ is a maximum for $\omega \tau=1$. The blocking temperature $T_{B}$ for such nanoparticles that do not interact can be found by setting $f=f_{m}$ to arrive at

$$
T_{B}=\frac{K_{a} V}{k_{B} \ln \left(f_{o} / f_{m}\right)}
$$

When the particles interact, Eq. 2.54 is modified to the Vogel-Fulcher equation (Shtrikman \& Wohlfarth, 1981; Shim, et al., 2006; Tholence, 1993):

$$
T_{B}=T_{o}+\frac{K_{a} V}{k_{B} \ln \left(f_{o} / f_{m}\right)}
$$

From Eq. 2.55, it can be seen that $T_{B}$ should increase with increasing $f_{m}$. By utilizing temperature dependent ac measurements at multiple $f_{m}$ values for each sample in this dissertation, the quantities $K_{a}$, $f_{o}$, and $T_{o}$ are determined in Chapters 4,5 , and 6 using the theoretical framework provided here. 


\section{Chapter 3}

\section{Synthesis and Structural Characterization of Maghemite Nanoparticles}

\subsection{Introduction}

This chapter contains information regarding the synthesis and structural characterization of oleic acid-coated $\gamma-\mathrm{Fe}_{2} \mathrm{O}_{3}$ nanoparticles. The synthesis procedure used is described and the modifications to produce the different sizes are discussed. The characterization of the particle diameters by transmission electron microscopy (TEM) is presented as well as the crystalline size estimates determined from X-ray diffraction (XRD). The oleic acid coating present on the nanoparticles is analyzed using infrared spectroscopy measurements and the oleic acid contribution to the mass of the samples is determined from thermogravimetric analysis (TGA). The information presented in this chapter will be used in Chapters 4 and 5 to aid in analyzing the magnetic data.

\subsection{Synthesis of Maghemite Nanoparticles with Oleic Acid Coating}

\subsubsection{Chemicals Used for Synthesis}

In the synthesis of oleic acid-coated $\gamma-\mathrm{Fe}_{2} \mathrm{O}_{3}$ nanoparticles, the following chemicals were used as received without further modification:

- $\quad$ iron pentacarbonyl (Sigma Aldrich Stock \#481718)

- trimetylamine N-oxide (Sigma Aldrich Stock \#317594)

- $\quad$ oleic acid (Fisher Stock \#A195)

- dioctyl ether (Sigma Aldrich Stock \#249599)

- $\quad$ toluene (Sigma Aldrich Stock \#179965)

- $\quad$ anhydrous ethanol (Fisher Stock \#A405F)

- lauric acid (Sigma Aldrich Stock \#W261408)

All steps in the synthesis procedure were performed in a fume hood. An argon atmosphere was maintained inside the apparatus during synthesis by supplying flowing ultra-high-purity argon (Matheson Trigas) through the top of the condenser via a needle-tipped rubber tube that was inserted through a rubber stopper. A second needle-tip was additionally inserted into the rubber stopper to allow the escape of gas when the pressure built due to heating. The condenser was cooled by slowly running tap water. The equipment required for synthesis included:

- round-bottomed flask

- condenser

- thermometer 
- heating pad

- $\quad$ syringes (small narrow-tipped for iron pentacarbonyl \& wide-tipped for the oleic acid)

- graduated cylinder

- scale

- $\quad$ strong permanent magnet(s)

- $\quad$ watch glass

\subsubsection{Procedure Used for Synthesis}

Maghemite nanoparticles were synthesized using a modification of the procedure of Hyeon et al. (2001; Dutta, et al., 2004). The apparatus used, shown in Fig. 3.1, consists of a round bottom flask, a heating pad, and a condenser. To synthesize the oleic acid-coated $\gamma-\mathrm{Fe}_{2} \mathrm{O}_{3}$ nanoparticles, first, oleic acid was added to $10 \mathrm{~mL}$ of dioctyl ether in a round bottom flask and the mixture was heated to $100{ }^{\circ} \mathrm{C}$ under an argon atmosphere. The amount of oleic acid used for each sample will be discussed later. Once the temperature of the mixture was stable at $100{ }^{\circ} \mathrm{C}, 200 \mu \mathrm{L}$ of iron pentacarbonyl $\left(\mathrm{Fe}(\mathrm{CO})_{5}\right)$ was drawn into a Teflon-tipped syringe. To add the $\mathrm{Fe}(\mathrm{CO})_{5}$ into the solution, the stopper holding the thermometer was briefly removed and the syringe inserted. Once the $\mathrm{Fe}(\mathrm{CO})_{5}$ was added, the iron oleate complex began to form and the stopper with the thermometer was quickly replaced while the solution changed from clear to a deep yellow and dense vapors began to form (Fig. 3.2). The resulting iron-oleate complex was subsequently heated to $285{ }^{\circ} \mathrm{C}$ and allowed to reflux for one hour during which the formation of iron nanocrystallites occurred. The heat was removed and the solution allowed to cool. To oxidize the iron particles, $0.34 \mathrm{~g}$ of dehydrated $\left(\mathrm{CH}_{3}\right)_{3} \mathrm{NO}$ was added to the mixture. The resulting solution was heated to and held at $130{ }^{\circ} \mathrm{C}$ for two hours. The temperature was then increased to $285{ }^{\circ} \mathrm{C}$ and the solution was

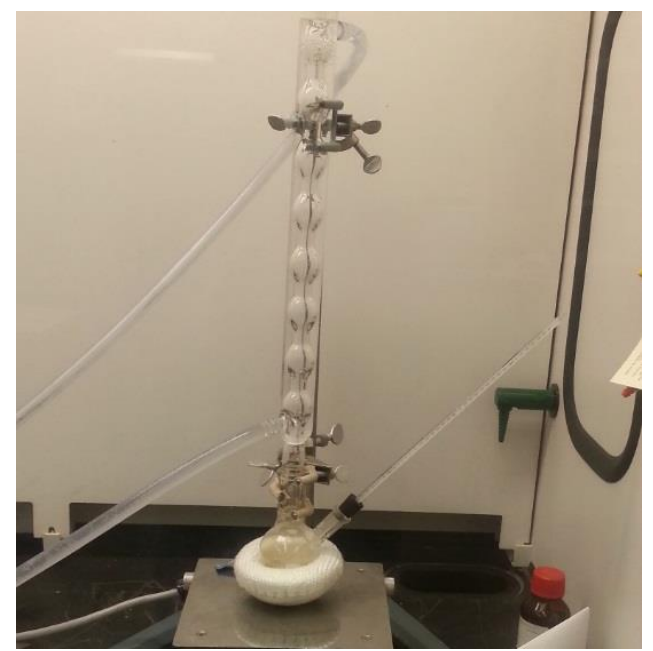

Figure 3.1: Synthesis apparatus used. Contents of the flask were heated under an argon atmosphere while temperature was monitored using a standard thermometer. 


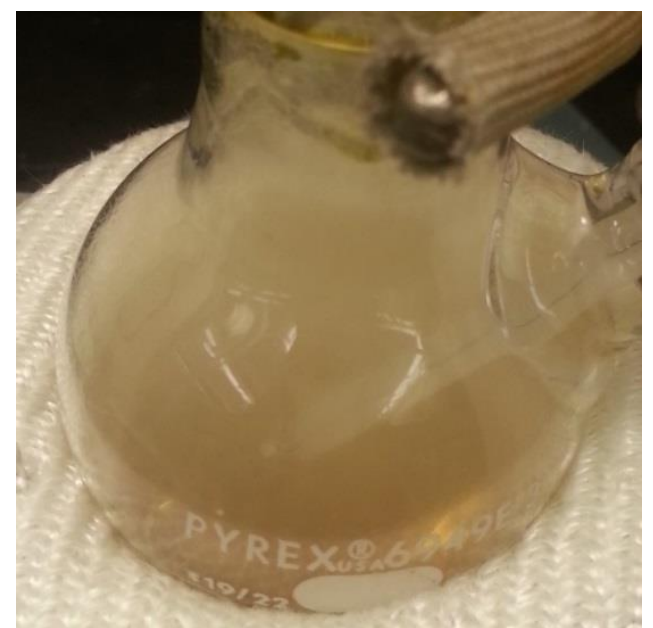

Figure 3.2: Solution after $\mathrm{Fe}(\mathrm{CO})_{5}$ was added and thermometer was returned. The stage at which the iron oleate complex is formed.

allowed to reflux for one hour. The result of the synthesis was a deep brown solution that contained $\gamma$ $\mathrm{Fe}_{2} \mathrm{O}_{3}$ nanoparticles coated in oleic acid.

The coated nanoparticles were removed from solution by adding small amounts of ethanol to $\sim 1$ $\mathrm{mL}$ aliquots of solution until the particles began to flocculate. The vial containing the aliquot was set on top of a strong permanent magnet and left there until all the particles settled out and the supernatant was almost completely clear. Keeping the magnet underneath the vial to hold the particles in place, the supernatant was poured out.

The particles were washed by first adding a few drops of toluene and then shaking the vial to disperse the nanoparticles. Then, a few drops of ethanol were added to cause the particles to flocculate. A magnet was placed under the vial to help expedite the process of settling out the particles and hold them in place while the supernatant was poured off. This process was repeated two additional times (total of three washing steps). The washed particles were collected by adding a small amount of ethanol into the vial and shaking to dislodge particles stuck at the bottom of the vial. The ethanol-nanoparticle solution was poured into a watch glass and the ethanol was allowed to evaporate. The dried powder was collected and used for magnetic measurements.

The ratio of oleic acid to iron pentacarbonyl was adjusted to control particle size; however, the size results obtained by Hyeon, et al. (2001) could not be reproduced. The results were consistent with those of Dutta, et al. (2004), though. Increasing concentrations of oleic acid in the solution lead to larger particles being formed as can be seen from Table 3.1. Increasing the oleic acid content beyond 1:3 did not increase particle size beyond $7 \mathrm{~nm}$. In fact; attempts to produce nanoparticles using a 1:4 ratio produced another $7.0 \mathrm{~nm}$ sample. In addition, increasing the reflux time on the 1:3 synthesis attempt 
Table 3.1: Synthesis parameters for $\gamma-\mathrm{Fe}_{2} \mathrm{O}_{3}$ nanoparticle samples. The quantity of $\mathrm{Fe}(\mathrm{CO})_{5}$ was the same $(200 \mu \mathrm{L})$ for each synthesis attempt and the molar ratio was varied by changing the amount of oleic acid $(O A)$ used. The mean particle diameter $\langle D\rangle$ has been determined from TEM.

\begin{tabular}{|c|c|c|}
\hline Ratio of $\mathrm{Fe}(\mathrm{CO})_{5}: \mathrm{OA}$ & $\mathrm{OA}(\mathrm{g})$ & $\langle D\rangle(\mathrm{nm})$ \\
\hline $2: 1$ & 0.21 & $2.5 \pm 0.7$ \\
\hline $1: 1$ & 0.43 & $3.4 \pm 0.7$ \\
\hline $1: 3$ & 1.28 & $6.3 \pm 0.6$ \\
\hline $1: 3$ & 1.28 & $7.0 \pm 0.8$ \\
\hline
\end{tabular}

yielded particles with $\langle D\rangle=6.2 \mathrm{~nm}$ and $\sigma_{D}=0.6 \mathrm{~nm}$, making it nearly indistinguishable from the $6.3 \mathrm{~nm}$ sample; therefore, the time consuming magnetic measurements were not carried out on this sample.

To further try to produce nanoparticles larger than $7.0 \mathrm{~nm}$, following another procedure described in Hyeon, et al. (2001), $0.2 \mathrm{~mL}$ of $\mathrm{Fe}(\mathrm{CO})_{5}$ was injected into a solution containing $0.91 \mathrm{~g}$ of lauric acid, 7 $\mathrm{mL}$ of dioctyl ether, and $0.57 \mathrm{~g}$ of $\left(\mathrm{CH}_{3}\right)_{3} \mathrm{NO}$ under an argon atmosphere at $100{ }^{\circ} \mathrm{C}$ while stirring and then continuing to stir the solution at $120{ }^{\circ} \mathrm{C}$ for 1 hour before heating to reflux for an additional hour. This attempt was unsuccessful. When ethanol was added to the resulting solution, nothing settled out even with the aid of a strong permanent magnet indicating that the solution did not contain magnetic nanoparticles.

One final synthesis attempt to produce $\gamma-\mathrm{Fe}_{2} \mathrm{O}_{3}$ nanoparticles larger than $7.0 \mathrm{~nm}$ was also based on a procedure from Hyeon, et al. (2001). The procedure followed to produce the $7.0 \mathrm{~nm}$ samples was repeated except after the iron oxide nanocrystallites were formed, an additional batch of iron oleate complex was added and the mixture and aged at $285{ }^{\circ} \mathrm{C}$ for one hour before removing the heat and allowing the solution to cool to room temperature. The iron particles were again oxidized by adding, 0.34 $\mathrm{g}$ of dehydrated $\left(\mathrm{CH}_{3}\right)_{3} \mathrm{NO}$ and heating to $130{ }^{\circ} \mathrm{C}$ for two hours. The temperature was then increased to $285^{\circ} \mathrm{C}$ and the solution was allowed to reflux for one hour. The resulting particles were not significantly larger than $7.0 \mathrm{~nm}$. After the numerous synthesis attempts failed to yield a nanoparticle sample significantly larger than $7.0 \mathrm{~nm}$, analysis was carried out using the $7.0 \mathrm{~nm}, 6.3 \mathrm{~nm}, 3.4 \mathrm{~nm}$, and $2.5 \mathrm{~nm}$ samples only. A flow diagram showing the synthesis procedure that ultimately resulted in the nanoparticle samples discussed can be seen in Fig. 3.3 with the octagonal sections indicating the formation of a product (iron oleate complex, iron nanocrystallites, and $\gamma-\mathrm{Fe}_{2} \mathrm{O}_{3}$ nanoparticles) for reference. 


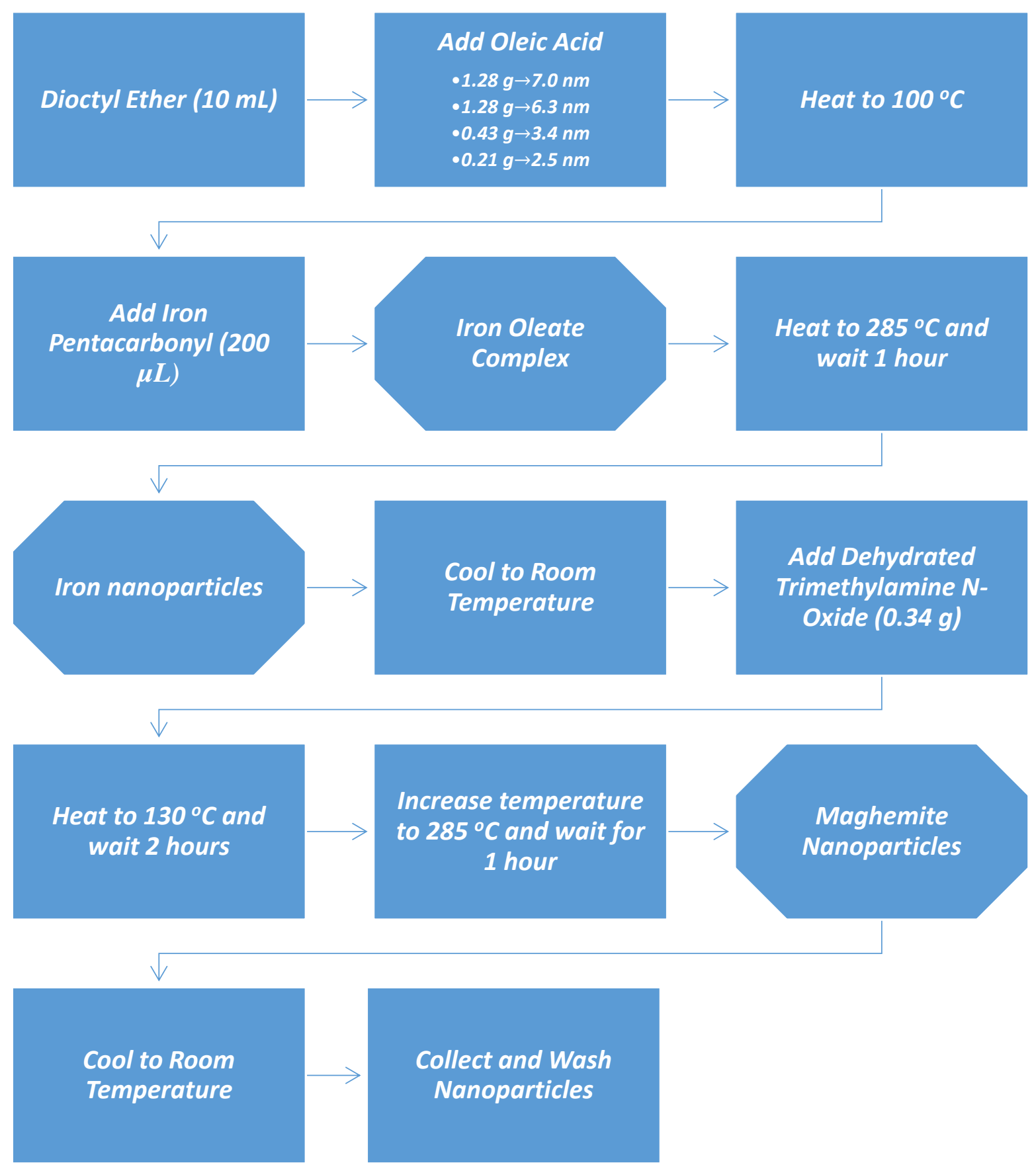

Figure 3.3: Synthesis procedure for $\gamma-\mathrm{Fe}_{2} \mathrm{O}_{3}$ nanoparticles. Each rectangular section indicates a step in the synthesis procedure and each octagonal section represents a product of the synthesis procedure. 


\subsection{Transmission Electron Microscopy}

Transmission electron microscopy (TEM) was used to determine the size and size distribution of the nanoparticles in each sample. Samples were prepared for TEM during washing. At the final washing step, after the particles had been suspended in toluene and dispersed by shaking, a pipette was used to collect several drops of solution. This was then dropped directly onto a 400 mesh carbon-coated copper TEM grid purchased from Ted Pella. The grid was allowed to dry for a minimum of 2 hours before TEM was performed. A JEOL JEM 2100 TEM was used with a $\mathrm{LaB}_{6}$ filament operated at $200 \mathrm{kV}$ and images were obtained using an ES500W Erlangshen CCD camera and Digital Micrograph software purchased from Gatan, Inc.

The TEM grid with the sample loaded on it was placed into a single-tilt sample holder provided by JEOL for use with the TEM and was loaded into the microscope. The TEM was aligned and operated at $200 \mathrm{kV}$. Briefly, the condenser aperture and the beam were centered at 50k magnification and the optical axis of the electron gun and condenser system were aligned at 100k magnification before the condenser lens astigmation was corrected and the condenser lens deflection coil tilt was adjusted as needed. The magnification was then increased to $120 \mathrm{k}$ and an image of the NPs was obtained and used to center the current axis and align the objective lens. The magnification was then increased to $300 \mathrm{k}$ and the image was used to center the voltage axis/align the objective lens. The objective lens astigmation was corrected using the $300 \mathrm{k}$ image by setting the CCD area to $1024 \times 1024$ pixels and performing a live fast Fourier transform of the digital image as the objective lens astigmation was adjusted until the Fourier transformed image was as round as possible. Once the microscope had been aligned, more than 20 images were taken of the sample to be used for the determination of the size distribution. For each sample, the magnetization and the number of images taken depended partly on the density of the particles on the grid but more than 300 NPs were measured even for the least dense $3.4 \mathrm{~nm}$ sample. Representative images can be seen in Fig. 3.4.

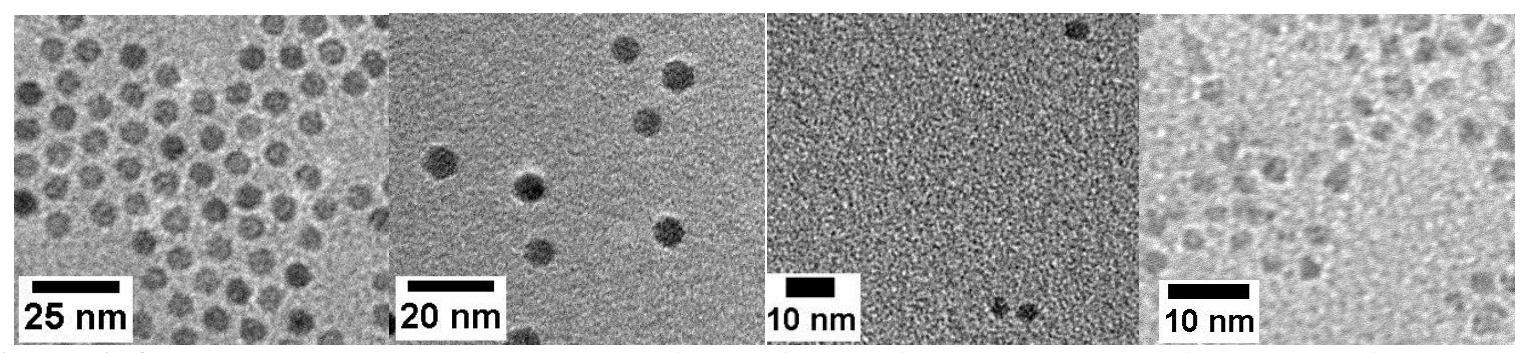

Figure 3.4: Representative TEM micrographs of the maghemite nanoparticles. From left to right, $\langle D\rangle=7.0 \mathrm{~nm}, 6.3 \mathrm{~nm}, 3.4 \mathrm{~nm}$, and $2.5 \mathrm{~nm}$. 
Table 3.2: Size distribution parameters determined from the fit of histograms in Fig. 3.4 to Eq. 3.1 and calculated quantities, $\langle D\rangle$ and $\sigma_{D}$ determined from Eq. 3.2 and 3.3, respectively.

\begin{tabular}{|c|c|c|c|}
\hline$\langle D\rangle(\mathrm{nm})$ & $\sigma_{D}(\mathrm{~nm})$ & $D_{o}(\mathrm{~nm})$ & $\lambda_{D}$ \\
\hline 2.5 & 0.7 & 2.4 & 0.28 \\
\hline 3.4 & 0.7 & 3.4 & 0.21 \\
\hline 6.3 & 0.6 & 6.2 & 0.09 \\
\hline 7.0 & 0.8 & 7.0 & 0.11 \\
\hline
\end{tabular}

The NP areas were determined from micrographs using the National Institutes of Health's, public domain ImageJ program and the analyze particles function. In ImageJ, the digital micrograph files were opened, and the threshold of each adjusted. When necessary, micrographs were broken up into regions over which uniform analysis could be performed. The software was set to fit particles to ellipses and display outlines of all measured particles. The outlines were visibly checked against the original micrograph to identify regions where more than one particle had been included together or only a partial

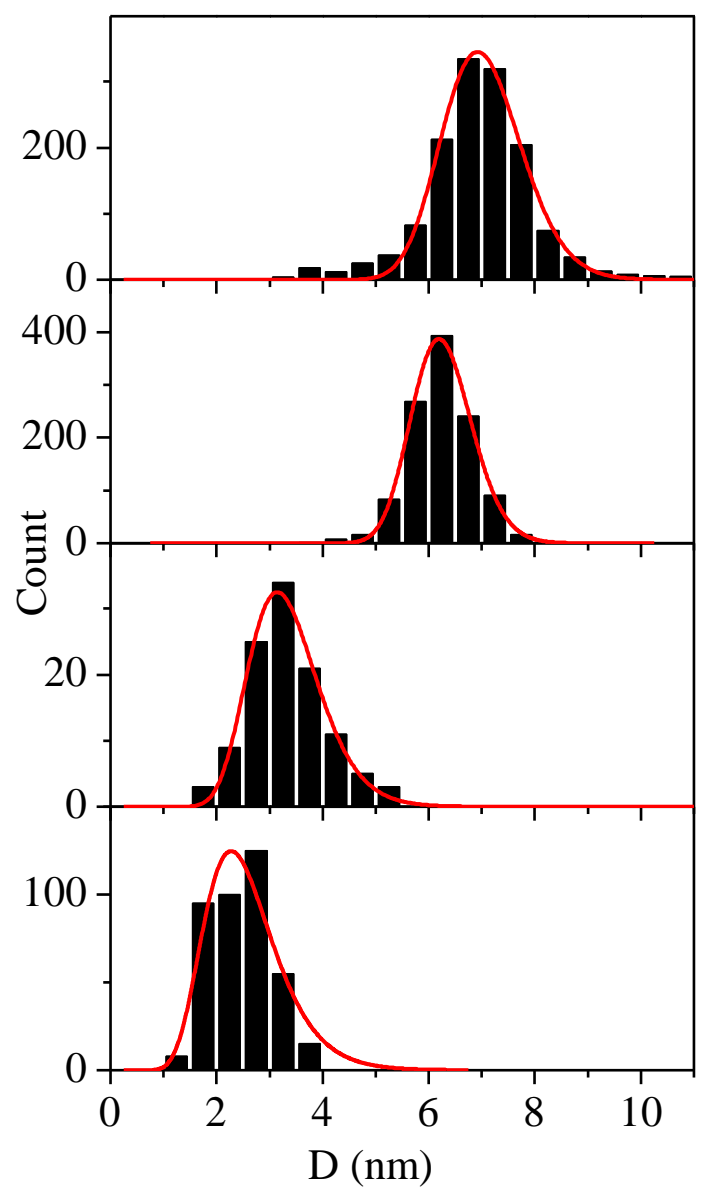

Figure 3.5: Size distributions of maghemite nanoparticles. The black bars represent the number of particles counted with the corresponding diameter and the red curve is a fit to Eq. 3.1. The parameters from the fits are given in Table 3.2. 
particle had been measured. These data were removed and the results were analyzed in Origin Lab software. The measured nanoparticle areas were used to calculate diameters assuming spherical particles using $D=\sqrt{4 A / \pi}$. The major and minor axes that were measured for each particle were used to evaluate the aspect ratios and confirm the roundness of the particles. The majority of nanoparticles had aspect ratios of less than $\sim 1.3$.

A histogram of the particle diameters was plotted and the results were fit to a lognormal distribution of sizes (Richardson \& Desai, 1976) described by

$$
f(D)=\frac{1}{\sqrt{2 \pi} D \lambda_{D}} \exp \left\{-\frac{\left[\ln \left(D / D_{o}\right)\right]^{2}}{2 \lambda_{D}{ }^{2}}\right\}
$$

(a restatement of Eq. 2.38) shown as a solid red curve in Fig. 3.5. The parameters obtained from the fit are given in Table 3.2. From the values of $D_{o}$ and $\lambda_{D}$, the mean diameters and standard deviations given in Table 3.2 were determined using

$$
\langle D\rangle=D_{o} \exp \left[\lambda_{D}^{2} / 2\right]
$$

and

$$
\sigma_{D}=\langle D\rangle\left[\exp \left(\lambda_{D}^{2}\right)-1\right]^{1 / 2}
$$

respectively. Eq. 3.1-3.3 are explained in more detail in Section 2.7.

\subsection{X-ray Diffraction}

$\mathrm{X}$-ray diffraction was used to estimate the size of the nanoparticles and confirm the formation of maghemite. A Rigaku RU-300 X-ray Diffractometer with $\mathrm{CuK}_{\alpha}$ radiation $(\lambda=0.15418 \mathrm{~nm})$ was used. In Fig. 3.6, the XRD pattern for a commercial maghemite powder (Alfa Aesar Stock \# 39951) is shown with the peaks indexed for reference. The pattern was obtained using a step size of $0.015^{\circ}$ and an integration time of 15 seconds per step to match the conditions that would be used for the maghemite nanoparticles. The full width at half-maximum was measured for each of the major peaks and these values used as an approximation of the instrumental broadening, $b$ for each peak.

The XRD patterns for the maghemite nanoparticles are shown in Fig. 3.7. The increase in broadening of the peaks with decreasing particle diameter is especially evident when comparing the pattern for the $3.4 \mathrm{~nm}$ and $6.3 \mathrm{~nm}$ particles. The crystallite size and the strain can be calculated from the Williamson-Hall relationship described by: 


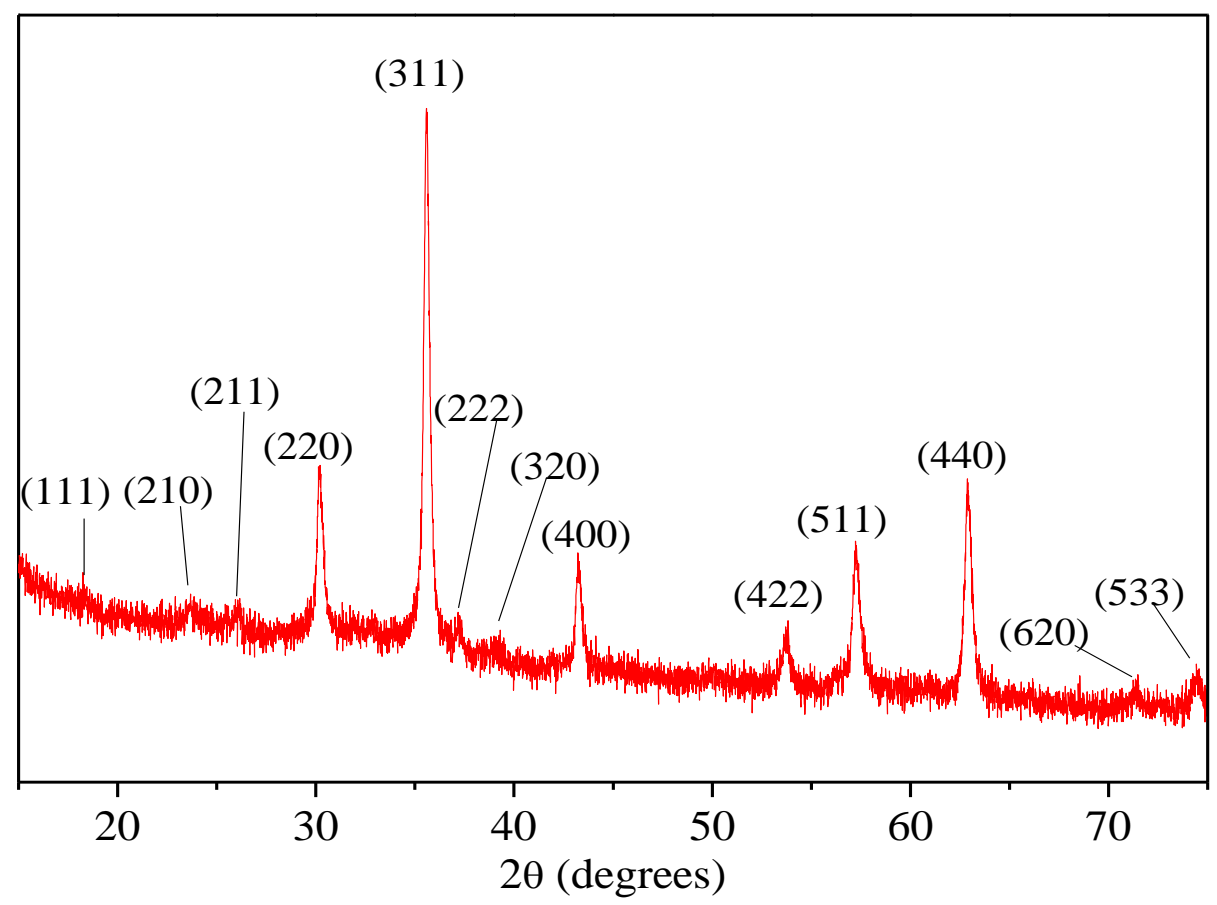

Figure 3.6: XRD pattern from commercial maghemite powder with indexed peaks. These data have been used to determine the instrumental broadening, $b$.

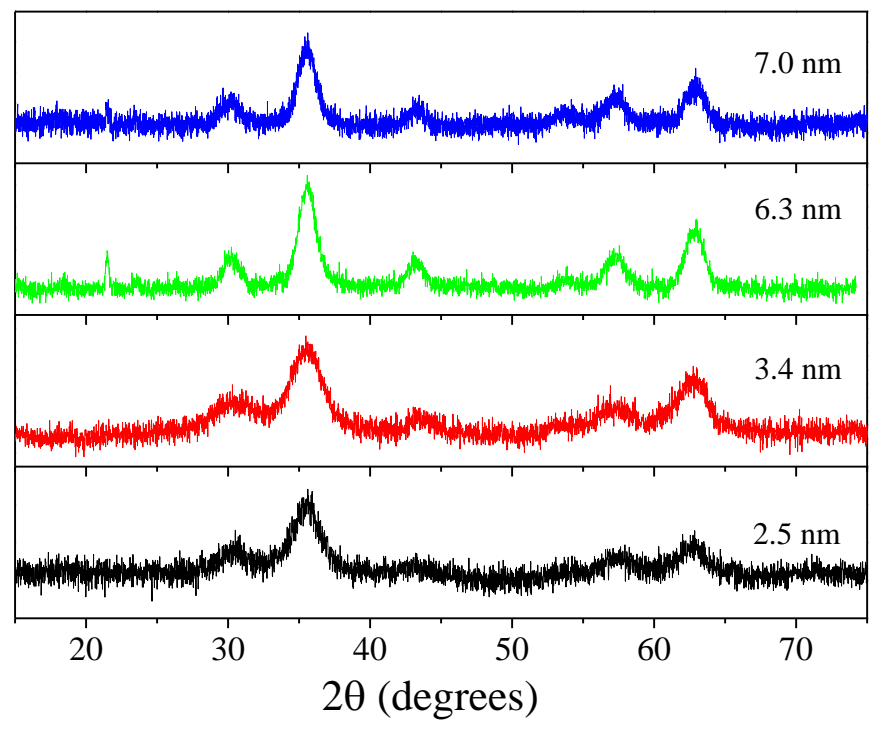

Figure 3.7: XRD data for maghemite nanoparticles with $\langle D\rangle=7.0 \mathrm{~nm}$ (blue), $6.3 \mathrm{~nm}$ (green), $3.4 \mathrm{~nm}$ (red), and $2.5 \mathrm{~nm}$ (black). All data were taken with a step size of 0.015 degrees with a measurement time of 15 seconds per step using a Rigaku RU-300 XRD. 


$$
\beta \cos \theta=\frac{0.98 \lambda}{D_{X R D}}+\eta \sin \theta
$$

where $\beta$ is peak broadening in radians, $\lambda$ is the wavelength of the $\mathrm{x}$-rays $\left(0.15418 \mathrm{~nm}\right.$ for $\mathrm{CuK}_{\alpha}$ radiation), $D_{X R D}$ is the crystalline diameter, and $\eta$ is the strain parameter. The peak broadening is given by $\beta=$ $\sqrt{B^{2}-b^{2}}$ where $B$ is the full width at half maximum of the peak and $b$ is the instrumental broadening determined from the XRD pattern of the bulk maghemite powder (Ibrahim, et al., 1992). The instrumental broadening $b$ is determined from the commercial powder since the size effects will be 1000 times smaller in the commercial micron-sized particles than the nanoparticles. The values of $b$ determined for each peak are $\sim 10-20 \%$ of the $B$ values determined for the nanoparticles so that $\beta$ is 97 -

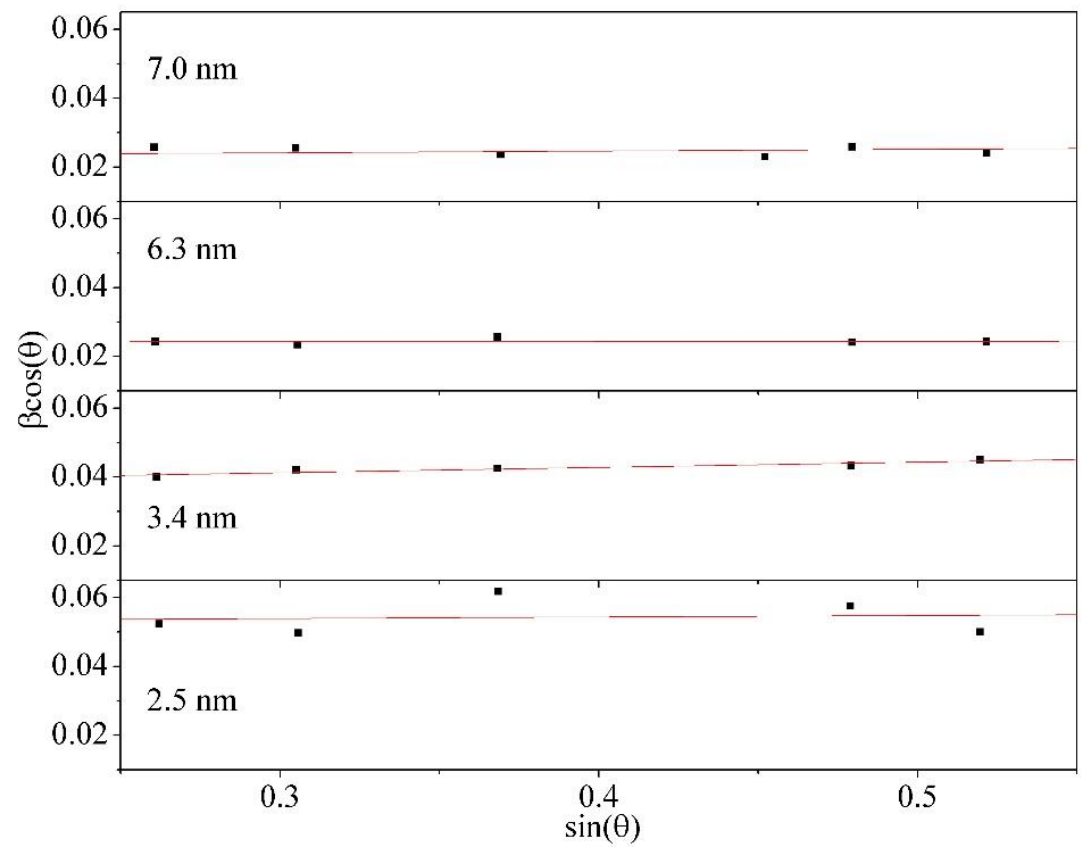

Figure 3.8: Data measured from the peaks in Fig. 3.7. Red line is Williamsen-Hall fit to Eq. 3.4 with the results given in Table 3.3.

Table 3.3: Results of Williamson-Hall (Eq. 3.4) fitting shown as solid red lines for each dataset in Fig. 3.8. The crystalline diameter from the fitting $\left(D_{X R D}\right)$ agrees well with the mean particle diameter $\langle D\rangle$ determined from TEM. The lattice parameters a calculated from the XRD peaks are consistent for all four samples.

\begin{tabular}{|c|c|c|c|}
\hline$\langle D\rangle(\mathrm{nm})$ & $D_{X R D}(\mathrm{~nm})$ & $\eta\left(10^{-3}\right)$ & $a(\mathrm{~nm})$ \\
\hline 7.0 & 6.7 & 5 & $0.842 \pm 0.002$ \\
\hline 6.3 & 6.3 & 0.7 & $0.840 \pm 0.001$ \\
\hline 3.4 & 4.1 & 15 & $0.840 \pm 0.002$ \\
\hline 2.5 & 2.9 & 4 & $0.842 \pm 0.002$ \\
\hline
\end{tabular}


99\% of $B$. To determine $D_{X R D}$ and $\eta$, a line was fit to a plot of $\beta \cos \theta$ vs. $\sin \theta$ and the slope taken to be $\eta$ while the intercept was taken to be $0.89 \lambda_{D} / D_{X R D}$ (Fig. 3.8). For extremely small particles, the effect of instrumental broadening is quite small as $B \gg b$, but for accurate comparison across all four samples, this effect has been included to determine the values of $D_{X R D}$ given in Table 3.3.

From the locations of the peaks in the XRD pattern of the maghemite nanoparticles, $d$ spacings were calculated using $d=\lambda / 2 \sin \theta$. For each $d$ spacing, the lattice parameter $a=\sqrt{h^{2}+k^{2}+l^{2}}$ was calculated where $h, k$, and $l$ are the miller indices associated with each peak. The values of $a$ for a given sample were plotted against $\cos ^{2} \theta / \sin \theta$ and the intercept of a linear fit to the data was used to determine $a$ for each sample given in Table 3.3. Any differences in the values of $a$ between samples are within the uncertainty of the values determined and so it is inferred that there is no significant change in the lattice constant with particle size for the four samples.

The values in Table 3.3 are in reasonable agreement with the mean diameters determined from TEM. Unfortunately, uncertainties in measuring the FWHM of the peaks, particularly for the smaller particles where some overlap between adjacent peaks may interfere with the shape, along with possible errors in removing the background and deviations between the true instrumental broadening and the broadening determined from our submicron particles makes accurate determination of these parameters difficult. It is reassuring, however, that the values determined are in reasonable agreement with those of TEM.

\subsection{Thermogravimetric Analysis}

Thermogravimetric analysis (TGA) is performed with a Q50 TGA from TA Instruments to determine the mass of magnetic material present in the samples and the approximate thickness of the oleic acid coating. For TGA, the particles are placed in a titanium pan that is suspended and the mass of the sample is determined. The pan is inserted into a furnace with dry flowing nitrogen gas and then is heated while the mass is continually measured (Fig. 3.9). As the temperature reaches the boiling point of the sample constituents, there is a change in the mass associated with the loss of the surfactants. Because there is little to no change in the mass of $\gamma-\mathrm{Fe}_{2} \mathrm{O}_{3}$ with temperature the mass left at the end is considered to be the mass due to the $\gamma-\mathrm{Fe}_{2} \mathrm{O}_{3}$. This information is used to scale the measured magnetization values to be in terms of magnetization per mass of magnetic material $\left(\gamma-\mathrm{Fe}_{2} \mathrm{O}_{3}\right)$. This is critical for true comparisons since the magnetic properties arrive from the $\gamma-\mathrm{Fe}_{2} \mathrm{O}_{3}$ and not from the oleic acid and comparisons of magnetic moment per total sample mass are not valid when the ratio of oleic acid to $\gamma$ $\mathrm{Fe}_{2} \mathrm{O}_{3}$ are not consistent from one sample to the next. 


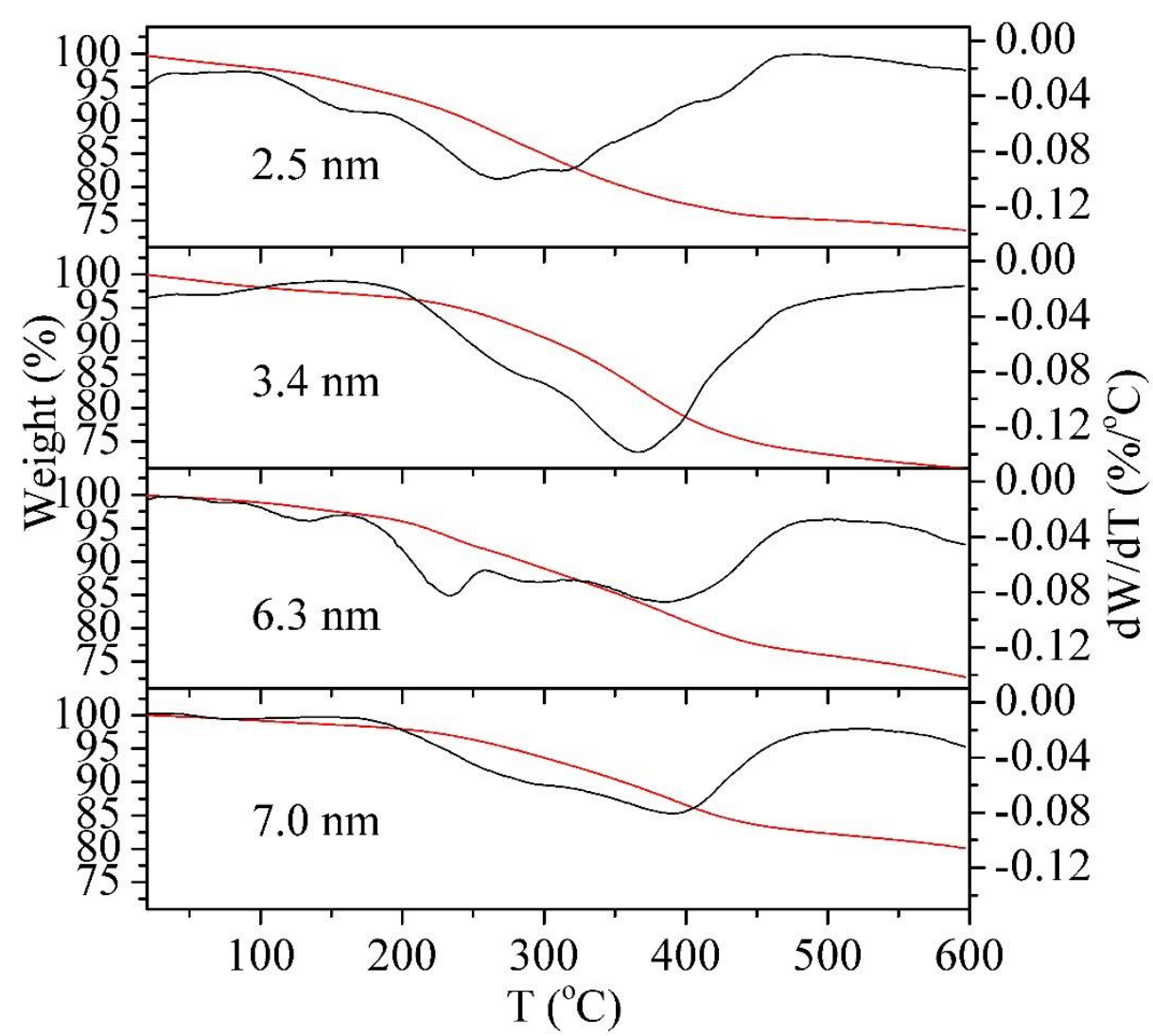

Figure 3.9: TGA results. Red curve shows the weight \% of the sample as a function of temperature and black curve shows a derivative of the weight vs Temperature curve. Each measurement was done by increasing the temperature at a rate of $5 \mathrm{C} / \mathrm{min}$. The results of TGA are summarized in Table 3.4.

In addition, determining the amount of oleic acid present in the sample allows estimation of the average interparticle spacing by assuming a relatively uniform oleic acid coating for each of the particles. If the particles are spherical and the coating thickness $t$ is the same for each particle, then the following relationship may be used to estimate the oleic acid thickness:

$$
\frac{m_{\text {initial }}}{m_{\text {final }}}=\frac{m_{m+o a}}{m_{m}}=\frac{\rho_{m} V_{m}+\rho_{o a} V_{o a}}{\rho_{m} V_{m}}
$$

where $\rho_{m}=4.856 \mathrm{~g} / \mathrm{cm}^{3}$ and $\rho_{o a}=0.895 \mathrm{~g} / \mathrm{cm}^{3}$ are the density of $\gamma-\mathrm{Fe}_{2} \mathrm{O}_{3}$ and oleic acid, respectively and $V_{m}$ and $V_{o a}$ are the volume of the $\gamma-\mathrm{Fe}_{2} \mathrm{O}_{3}$ and the oleic acid, respectively. Then, solving for the thickness of the shell gives

$$
t=\frac{\langle D\rangle}{2}\left\{\sqrt[3]{\frac{\rho_{m}}{\rho_{\text {oa }}}\left[\frac{m_{\text {initial }}}{m_{\text {final }}}-1\right]+1}-1\right\}
$$

where the ratio $m_{\text {initial }} / m_{\text {final }}=1 / r$ is determined from the TGA measurements, and $D$ is the diameter of the $\gamma-\mathrm{Fe}_{2} \mathrm{O}_{3}$ particle $\left(\langle D\rangle+2 t\right.$ is the diameter of the oleic acid-coated $\gamma-\mathrm{Fe}_{2} \mathrm{O}_{3}$ nanoparticle). In this 
Table 3.4: Results from TGA analysis. The ratio of the mass of maghemite $\left(m_{m}\right)$ to the measured sample mass (maghemite + oleic acid, $m_{m+o a}$ ) and oleic acid coating thickness $t$ calculated from Eq. 3.6.

\begin{tabular}{|c|c|c|}
\hline$\langle D\rangle(\mathrm{nm})$ & $r=m_{m} / m_{m+o a}$ & $t(\mathrm{~nm})$ \\
\hline 2.5 & .749 & 0.5 \\
\hline 3.4 & .720 & 0.8 \\
\hline 6.3 & .757 & 1.3 \\
\hline 7.0 & .820 & 1.0 \\
\hline
\end{tabular}

way, an estimate for the thickness of the oleic acid coating can be determined from the TGA results by knowing the mean diameter of the particle (results in Table 3.4).

\subsection{Fourier Transform Infrared Spectroscopy}

Fourier Transform Infrared Spectroscopy (FTIR) was carried out on the $2.5 \mathrm{~nm}$ and $6.3 \mathrm{~nm}$ samples using an Infinity Gold FTIR from Thermo Mattson to determine how the oleic acid was bound to the maghemite nanoparticles. For the pure oleic acid, commercial maghemite, and $7.0 \mathrm{~nm}$ particles, a Perkin Elmer Spectrum 100 FTIR Spectrometer was used. Fig. 3.10 shows the FTIR spectra for pure oleic acid, commercial $\gamma-\mathrm{Fe}_{2} \mathrm{O}_{3}$ powder, and the $7.0 \mathrm{~nm}$ nanoparticles as synthesized. For the $7.0 \mathrm{~nm}$ spectrum, there are broad peaks around $1644-1520 \mathrm{~cm}^{-1}$ and $1461-1313 \mathrm{~cm}^{-1}$. From these peaks, possible $\Delta=$

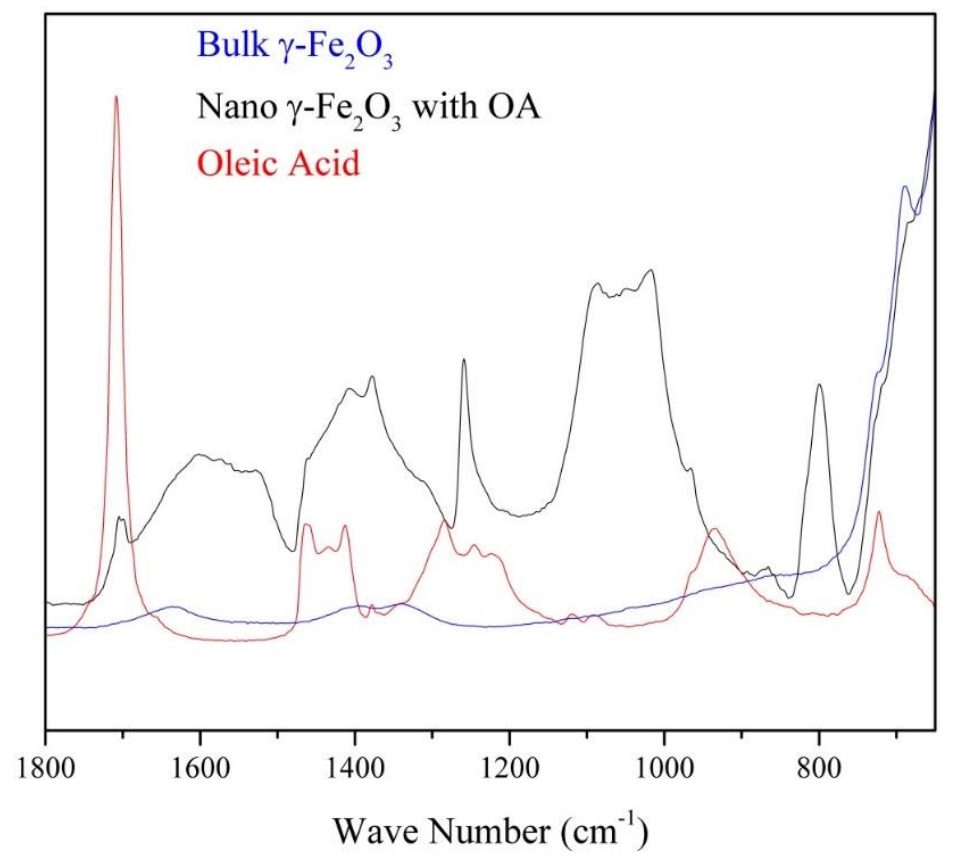

Figure 3.10: FTIR of bulk, commercial $\gamma-\mathrm{Fe}_{2} \mathrm{O}_{3}$ (blue), $7.0 \mathrm{~nm} \gamma-\mathrm{Fe}_{2} \mathrm{O}_{3}$ nanoparticles coated in oleic acid (black) and pure oleic acid (red). 


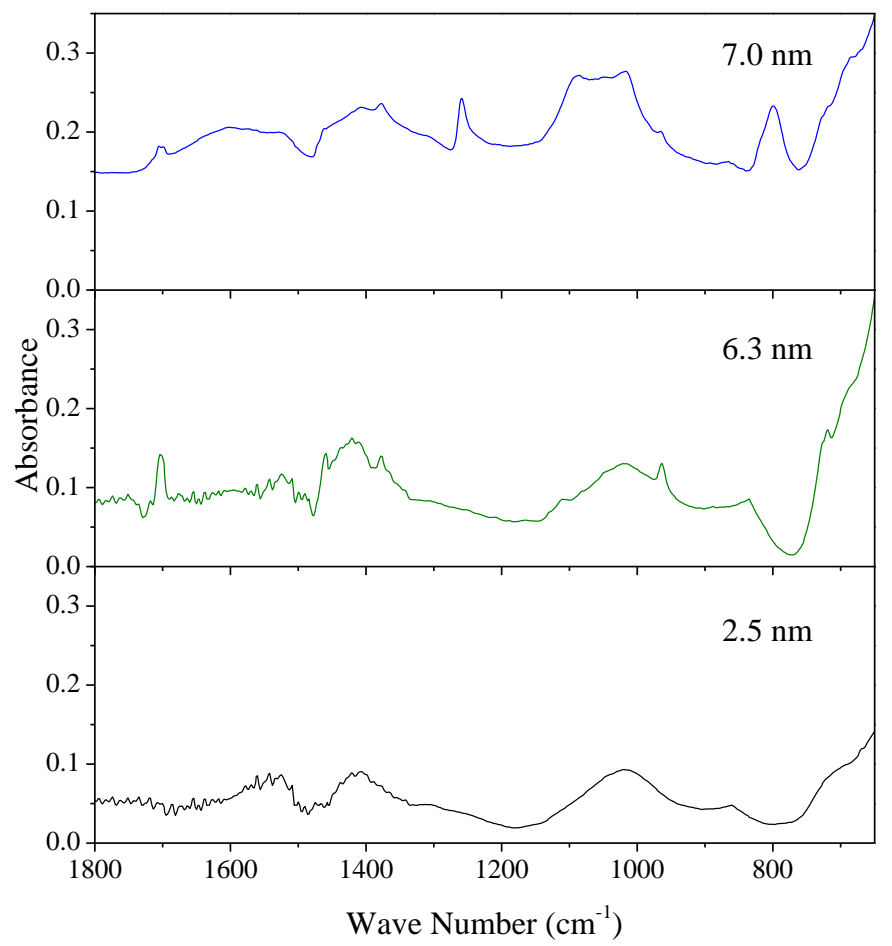

Figure 3.11: FTIR of $7.0 \mathrm{~nm}$ particles (top), $6.3 \mathrm{~nm}$ particles (center), and $2.5 \mathrm{~nm}$ particles (bottom) $v_{a}\left(\mathrm{COO}^{-}\right)-v_{S}\left(\mathrm{COO}^{-}\right)$values from $59-331 \mathrm{~cm}^{-1}$ can be calculated indicating that multiple types of metal-oleic acid complexes may be present on the nanoparticle surface including the unidentate $(\Delta>200$ $\mathrm{cm}^{-1}$ ), bidentate $\left(\Delta<100 \mathrm{~cm}^{-1}\right)$, and bridging complexes (intermediate values of $\Delta$ ) (Nakamoto, 1986). The peak near $1710 \mathrm{~cm}^{-1}$ is also present in pure oleic acid and is associated with the carbonyl stretching mode $(\mathrm{C}=\mathrm{O})$ and may indicate that some of the oleic acid is not bound via covalent bonds, but rather via hydrogen bonds (Soler, et al., 2007).

In Fig. 3.11, the FTIR spectra for each of the nanoparticle samples is shown. For the $6.3 \mathrm{~nm}$ sample, the possible values of $\Delta$ range from $30-212 \mathrm{~cm}^{-1}$ and for the $2.5 \mathrm{~nm}$ sample, the values of $\Delta$ range from $52-243 \mathrm{~cm}^{-1}$ indicating that, like for the $7.0 \mathrm{~nm}$ particles, the unidentate, bidentate and bridging complexes may all be present. The peak at $1710 \mathrm{~cm}^{-1}$ is more pronounced in the $6.3 \mathrm{~nm}$ spectrum than the $7.0 \mathrm{~nm}$ spectrum in agreement with the TGA findings that there is additional oleic acid on the $6.3 \mathrm{~nm}$ samples. This peak is absent in the $2.5 \mathrm{~nm}$ sample indicating that $\mathrm{t}$

here is no oleic acid hydrogen bonded to the nanoparticles in agreement with the thinner oleic acid coating estimate of only $0.5 \mathrm{~nm}$ for the $2.5 \mathrm{~nm}$ sample. FTIR data were not obtained for the $3.4 \mathrm{~nm}$ sample before TGA was performed; therefore, there is no data for the $3.4 \mathrm{~nm}$ sample in Fig. 3.11. 


\subsection{Summary}

In this chapter, the synthesis procedure used has been described and the structural characterization of the nanoparticle samples has been discussed. Results of TEM, XRD, FTIR, and TGA have been presented and have shown that oleic acid-coated $\gamma-\mathrm{Fe}_{2} \mathrm{O}_{3}$ nanoparticles with diameters $\langle D\rangle=7.0 \pm 0.8 \mathrm{~nm}, 6.3 \pm 0.6 \mathrm{~nm}, 3.4 \pm 0.8 \mathrm{~nm}, 2.5 \pm 0.7 \mathrm{~nm}$ have been produced with oleic acid coating thicknesses $t=1.0 \mathrm{~nm}, 1.3 \mathrm{~nm}, 0.8 \mathrm{~nm}$, and $0.5 \mathrm{~nm}$, respectively. The values of $D_{X R D}$ consistent with $\langle D\rangle$ determined from TEM provide evidence for the high degree of crystallinity of the nanoparticles. 


\section{Chapter 4}

\section{Magnetic Properties of 7.0 nm Maghemite Nanoparticles}

\subsection{Introduction}

In this chapter, magnetic measurements of $7.0 \mathrm{~nm}$ maghemite $\left(\gamma-\mathrm{Fe}_{2} \mathrm{O}_{3}\right)$ nanoparticles and the results and analysis of these measurements are discussed in considerable detail. The techniques and analysis in this chapter will form the basis of the work that will be presented in Chapter 5 . The magnetization $M$ of the sample was measured using an ac measurement system (ACMS) option for the Physical Property Measurement System (PPMS) purchased from Quantum Design, Inc. The data presented in this chapter are all from a single sample of $\gamma-\mathrm{Fe}_{2} \mathrm{O}_{3}$ nanoparticles coated with oleic acid that were being investigated in the Basic Pharmaceutical Sciences Department at WVU for potential uses in targeted drug delivery. The detailed characterization was carried out in part to aid collaborators with this larger project. The results presented in this chapter on the $7.0 \mathrm{~nm}$ maghemite sample have been published by the author recently (Pisane, et al., 2015) and presented at the 2014 American Physical Society March Meeting.

\subsection{Procedures for Magnetic Measurements}

In this section, the procedures used for carrying out the magnetic measurements on the $7.0 \mathrm{~nm}$ sample are described. The same procedures were used for magnetic measurements on all the other samples, results of which are presented in Chapter 5 and Chapter 6. Consequently, description of these procedures is not repeated there.

\subsubsection{Preparation and Loading of the Sample for Magnetic Measurements}

To prepare a synthesized sample for loading into the PPMS for magnetic measurements, the following procedure was adopted for all the samples investigated in this dissertation. After collecting the dried powder resulting from the washing procedure outlined in Chapter 3 (Section 3.1), the sample was funneled slowly into a plastic sample bag that was sealed on three sides using weighing paper and a pipette (Fig. 4.1). To minimize the amount of sample that clings to the inside of the pipette and the chances of clogging, the pipette was scored and most of the narrow end was removed. Once the bag was filled with at least $10 \mathrm{mg}$ of powder, the powder was pressed down into the bottom of the bag so that the powder was as compact as possible without breaking the existing seal on the plastic. This was done to ensure that the powder would not move during measurements and the bag was subsequently sealed on the open side. The completely sealed samples were stitched into drinking straws supplied by Quantum 
A

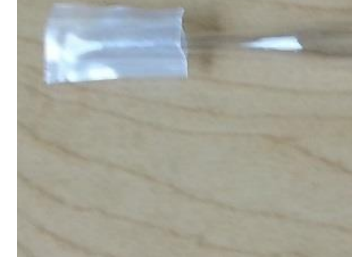

Figure 4.1: Sample preparation apparatus. A) sample bag B) shortened pipette C) weighing paper funnel

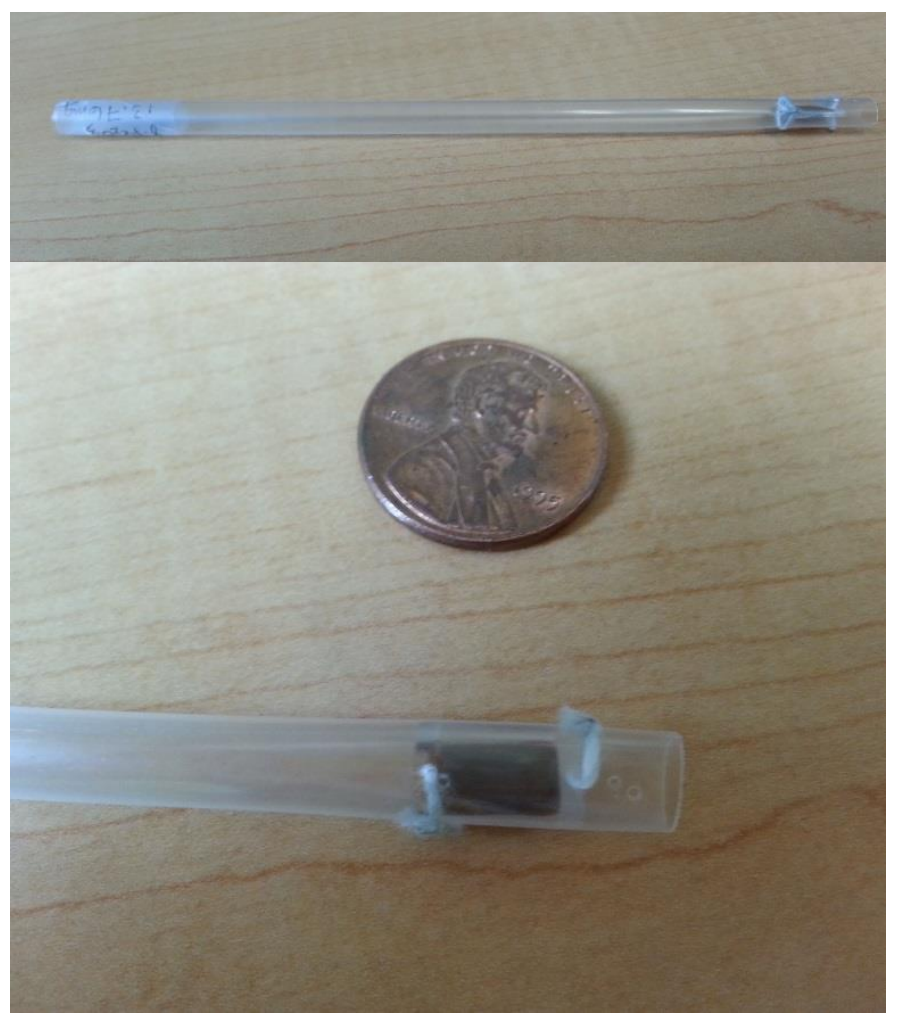

Figure 4.2: A representative ACMS sample. Top: a full sample as would be mounted in the ACMS Bottom: Up-close look at the magnetic sample stitched into the straw (penny shown for scale).

Design, Inc. for use with the ACMS using $100 \%$ cotton white embroidery thread to hold the sample in place during the measurements (Fig. 4.2).

\subsubsection{Mass and Background Determination}

In order to determine the magnetization in units of emu/g, it is necessary to know the mass of the sample accurately and to identify any contribution from the sample holder. Here, the weight of the samples was determined using an Ohaus Analytical Plus Balance to an accuracy of $\pm 0.1 \mathrm{mg}$. In order to 
determine the background signal, a stack of small pieces of the plastic material that holds the sample powder was stitched into a straw with the same $100 \%$ cotton thread used for the $\gamma-\mathrm{Fe}_{2} \mathrm{O}_{3}$ samples. The magnetization of the plastic was measured from 0 to $90 \mathrm{kOe}$ at $50 \mathrm{~K}$. The slope of the linear response was measured and a susceptibility of $\chi=-9.80 \times 10^{-7} \mathrm{emu} / \mathrm{g}$ Oe was determined. Because diamagnetic susceptibilities are temperature independent, the background signal is the same for every measurement made on a sample at a given magnetic field. The largest possible response will be for the maximum field of $90 \mathrm{kOe}$ where $M=-0.088 \mathrm{emu} / \mathrm{g}$. For each sample, the mass of the plastic used fell between 20 and 30 $\mathrm{mg}$ so that the largest possible contribution from the plastic was $-0.0026 \mathrm{emu}$ at $90 \mathrm{kOe}$. This is less than $0.5 \%$ of the smallest magnetic response measured at $90 \mathrm{kOe}$ for any sample in this dissertation. Any background signal from the sample holder falls within the uncertainty of the measurement, including the measurements of the mass of the samples; therefore, it was considered unnecessary to apply the background correction to the observed magnetization of the samples.

\subsection{3 ac Measurement System of PPMS}

All magnetic measurements in this dissertation were made with an ac measurement system (ACMS). The ACMS is both a dc magnetometer and an ac susceptometer. The temperature and dc magnetic field (up to $\pm 90 \mathrm{kOe}$ ) are controlled by the PPMS while the ACMS insert contains the drive coils for the ac magnetic field as well as the detection coils and a thermometer located near the sample. The sample sits in the center of the ACMS coilset on a long rod which is moved up and down through the coilset by the ACMS transport assembly which is located at the top of the PPMS sample chamber.

When a measurement is made, the ACMS transport assembly moves the sample through the pickup coils and the response is recorded. For dc measurements, all fields are applied using the superconducting magnet of the PPMS and each sample translation takes approximately 0.05 seconds. For all dc measurements presented in this dissertation, 5 individual scans of $M$ were averaged to generate each data point to reduce any contributions from random noise. For the ac measurements, the ac field was supplied by the ACMS drive coil. The ACMS drive coil can produce magnetic field amplitudes from 1 to $17 \mathrm{Oe}$ at frequencies ranging from $10 \mathrm{~Hz}$ to $10 \mathrm{kHz}$. Due to the effects of Eddy current heating that occur at high amplitudes and high frequencies, it was determined that 5 Oe amplitudes at frequencies up to 5 $\mathrm{kHz}$ provided the most useful results for the studies, though the original measurements for the $7.0 \mathrm{~nm}$ sample presented in this chapter were carried out at 10 Oe field amplitudes and the sample destroyed for TGA before 5 Oe measurements could be performed.

\subsubsection{Measurement Programming}

Both ac and dc measurement sequences were programmed and automated in MultiVu software. For each measurement, the temperature was stabilized prior to data acquisition as were any dc magnetic 
fields applied. Measurements that did not require the application of large magnetic fields were performed before those that did to reduce the total time spent demagnetizing the setup between measurements. Measurement sequences were chained so that when one finished, the next started automatically and the data collection was monitored remotely to allow for a large number of measurements to be made over the course of several days. Typical sequence files for $\mathrm{dc}$ and ac measurements are shown in the Appendix.

\subsubsection{Zero Field-Cooled and Field-Cooled Measurements}

The measurements of magnetization under the zero field-cooled (ZFC) and field-cooled (FC) conditions as a function of temperature were done using the following procedure. First, the sample was cooled to $2 \mathrm{~K}$ without any applied field to perform the ZFC measurement (data shown in Fig. 4.3). Because there was no applied magnetic field, the magnetic moments of the particles were randomly oriented when the sample was cooled to low temperatures. After reaching $2 \mathrm{~K}$, a small measuring field of 100 Oe was applied and the magnetization was measured as the temperature was increased. Since most of the particle magnetic moments were randomly oriented, the net magnetization of the sample was only $\sim 6$ $\mathrm{emu} / \mathrm{g}$ at $2 \mathrm{~K}$. As the temperature increased, particles began to have enough thermal energy to overcome the barrier of switching the direction of magnetization and rotate their moments to align with the magnetic field. This is why there is an initial increase in the magnetization with increasing temperature for the ZFC curve. After a certain point, the particles have gained enough thermal energy to overcome the barrier to switching between the parallel and antiparallel state and the net magnetization decreases as the particles become superparamagnetic. The peak in the ZFC magnetization occurs at the blocking temperature, $T_{B}=$ $35 \mathrm{~K}$. The width of the peak in the ZFC data is most likely due to the distribution in particle sizes as will

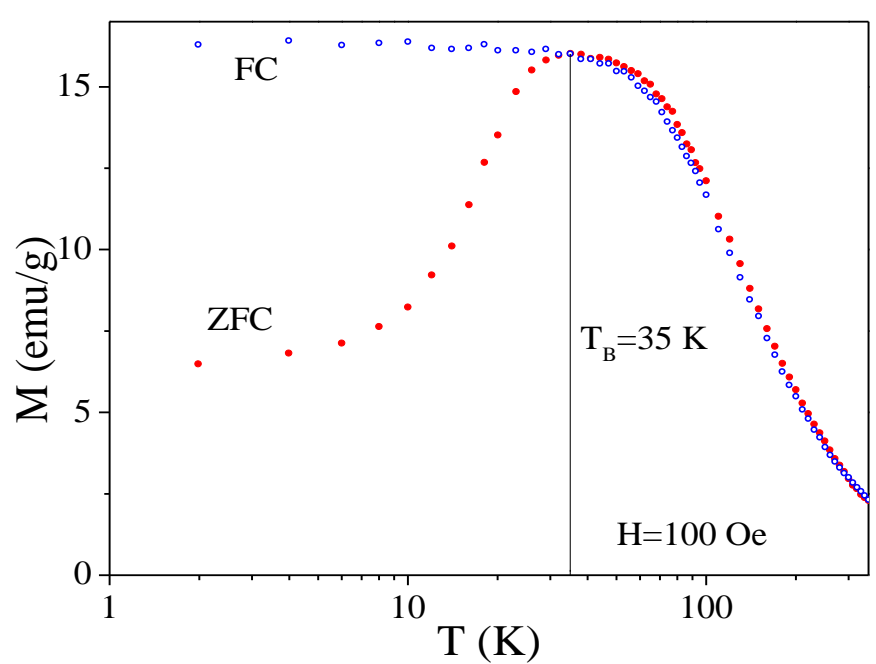

Figure 4.3: Magnetization of $7.0 \mathrm{~nm}$ maghemite nanoparticles vs. temperature with a 100 Oe measuring field for the ZFC and FC cases. 
be discussed in Section 4.4.5.

For the FC case, data were taken with $H=100$ Oe applied while decreasing the temperature. At high temperatures, the ZFC and FC curves are nearly identical but as the temperature decreases, the FC and ZFC cases bifurcate near $T_{B}$. The presence of the 100 Oe field while cooling is enough to keep the particle moments aligned so that, as the temperature was lowered, the net magnetization was frozen in and remained high as the temperature went to $2 \mathrm{~K}$. This general procedure was used for the ZFC and FC conditions for all the samples investigated in this dissertation.

\subsection{Temperature and Magnetic Field Dependence of Magnetization for the $7.0 \mathrm{~nm} \gamma-\mathrm{Fe}_{2} \mathrm{O}_{3}$ Sample}

\subsubsection{Magnetization below $T_{B}$}

Below $T_{B}=35 \mathrm{~K}$ the nanoparticles should behave ferrimagnetically and above $T_{B}$ the nanoparticles should behave superparamagnetically. To investigate the magnetic response to an applied magnetic field for $T<T_{B}$, hysteresis measurements were performed by first cooling the sample in zero applied magnetic field and then measuring $M$ from $90 \mathrm{kOe}$ to $-90 \mathrm{kOe}$ and back. The resulting curve for the $7.0 \mathrm{~nm} \gamma-\mathrm{Fe}_{2} \mathrm{O}_{3}$ sample at $T=2 \mathrm{~K}$ is shown in Fig. 4.4.

From the expanded view of the low-field region shown in the inset of Fig. 4.4, it is evident that the coercive field, $H_{C}$ is practically negligible even for $2 \mathrm{~K}$ in agreement with the observations of Dutta, et al. (2004) on similarly prepared $7 \mathrm{~nm} \gamma-\mathrm{Fe}_{2} \mathrm{O}_{3}$ nanoparticle samples. It is normal for $H_{C}$ to decrease as the temperature approaches $T_{B}$; however, the inset of Fig. 4.5 indicates that $H_{C}$ increases with

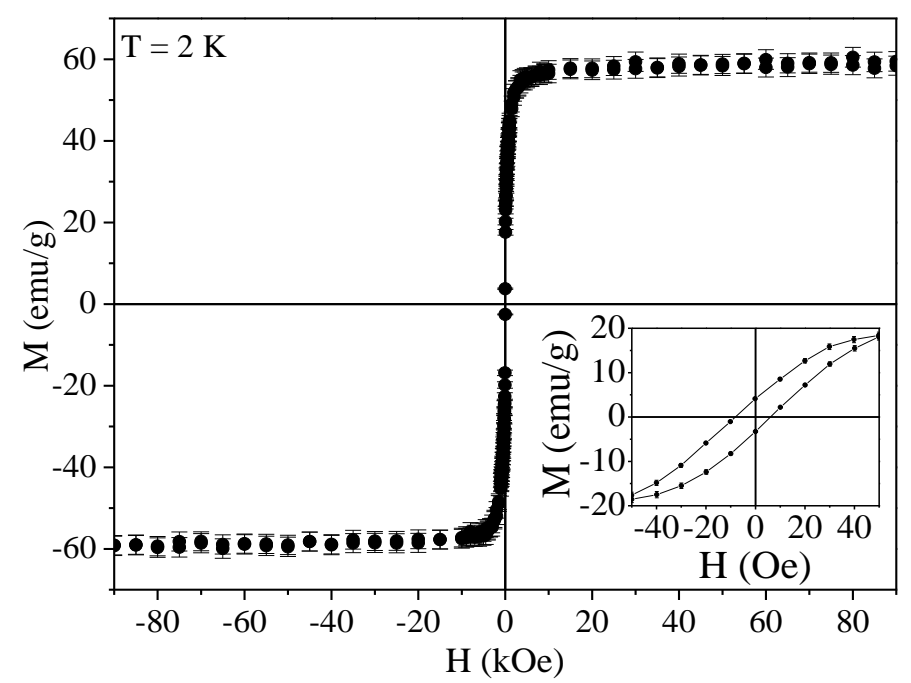

Figure 4.4: Hysteresis loop at $2 \mathrm{~K}$. Inset: the zoomed view for small $\mathrm{H}$ values shows $H_{C} \sim 10$ Oe. 


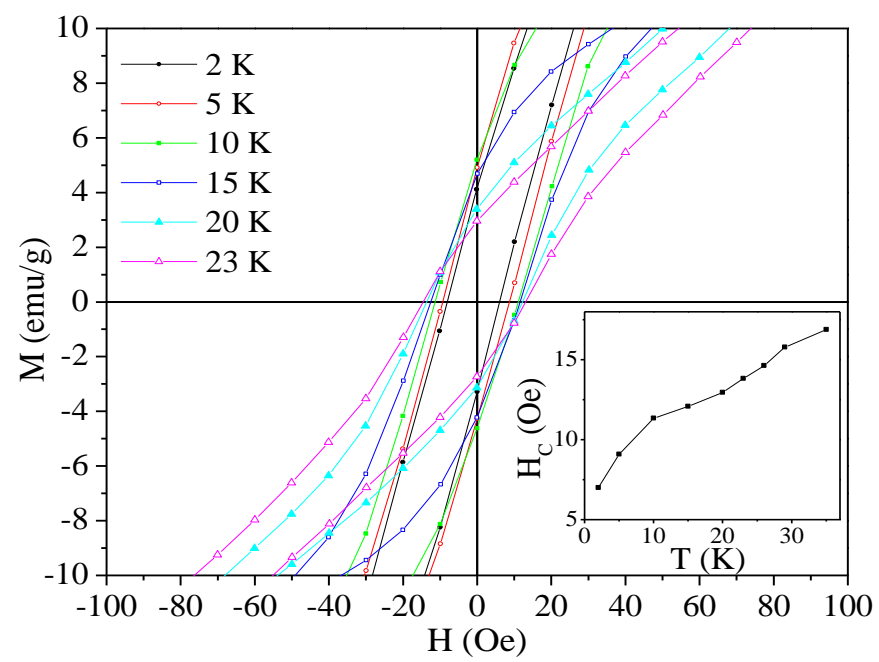

Figure 4.5: Close up view of the hysteresis loops taken for select temperatures. The hysteresis is plotted in the inset as a function of temperature.

increasing $T$. Because the hysteresis loops were taken one after the other by increasing the temperature only slightly between consecutive measurements, this anomalous increase in $H_{C}$ is attributed to an increase in the uncertainty of setting the field to zero for each consecutive measurement. For samples that followed, greater care was taken to remove these effects by increasing the temperature to $200 \mathrm{~K}$ and setting the field to zero by oscillating between positive and negative fields with decreasing magnitude between each consecutive measurement. In general, magnitudes of coercivity below about 20 Oe are considered to be practically zero because of the difficulty of setting the magnetic field to zero.

\subsubsection{Magnetization for $T>T_{B}$}

For temperatures $T>T_{B}$, the nanoparticles should be in the superparamagnetic state and the $M$ vs. $H$ curve should be described by the modified Langevin function (Makhlouf, et al., 1997; Punnoose, et al., 2005):

$$
\mathrm{M}=M_{o} L\left(\frac{\mu_{p} H}{k_{B} T}\right)+\chi_{a} H
$$

For reference, this is identical to Eq. 2.37 and is repeated here for ease in the discussion. To test whether the nanoparticles are in the superparamagnetic state, $M$ vs $H$ at $50 \mathrm{~K}, 100 \mathrm{~K}, 150 \mathrm{~K}, 200 \mathrm{~K}, 250 \mathrm{~K}$, and $300 \mathrm{~K}$ has been measured with the results shown in Fig. 4.6. The results of the fits to Eq. 4.1 are given in Table 4.1 for all but the $50 \mathrm{~K}$ measurement. Due to the poor fit obtained using Eq. 4.1 on the $50 \mathrm{~K}$ data, it was determined that $50 \mathrm{~K}$ was not sufficiently above $T_{B}$ to apply the modified Langevin equation, probably due to the size distribution of the nanoparticles which will keep the larger particles in the distribution blocked at $50 \mathrm{~K}$. For the samples that will be presented later, the $50 \mathrm{~K}$ isothermal data of $M$ vs. $H$ are also not fit to the modified Langevin equation for this reason. 
Table 4.1: Magnitudes of the parameters obtained from the Modified Langevin (Eq. 4.1) fit to the data of Fig. 4.6. $R^{2}$ provides a measure of the quality of the fit to the data with $R^{2}=1$ representing a perfect fit. The numbers in parenthesis are estimated uncertainties.

\begin{tabular}{|c|c|c|c|c|}
\hline$T(\mathrm{~K})$ & $M_{o}(\mathrm{emu} / \mathrm{g})$ & $\chi_{a\left(10^{-5} \mathrm{emu} / \mathrm{gOe}\right)}$ & $\mu_{p}\left(\mu_{B}\right)$ & $\mathrm{R}^{2}$ \\
\hline 100 & $53.2 \pm 0.4$ & $4.42 \pm 1.05$ & $7836 \pm 268$ & 0.9925 \\
\hline 150 & $51.6 \pm 0.4$ & $3.61 \pm 1.07$ & $7388 \pm 151$ & 0.9983 \\
\hline 200 & $49.8 \pm 0.4$ & $3.56 \pm 1.11$ & $7290 \pm 152$ & 0.9984 \\
\hline 250 & $50.7 \pm 0.4$ & $3.46 \pm 0.98$ & $7325 \pm 131$ & 0.9990 \\
\hline 300 & $47.2 \pm 0.3$ & $3.40 \pm 0.86$ & $6823 \pm 117$ & 0.9993 \\
\hline
\end{tabular}

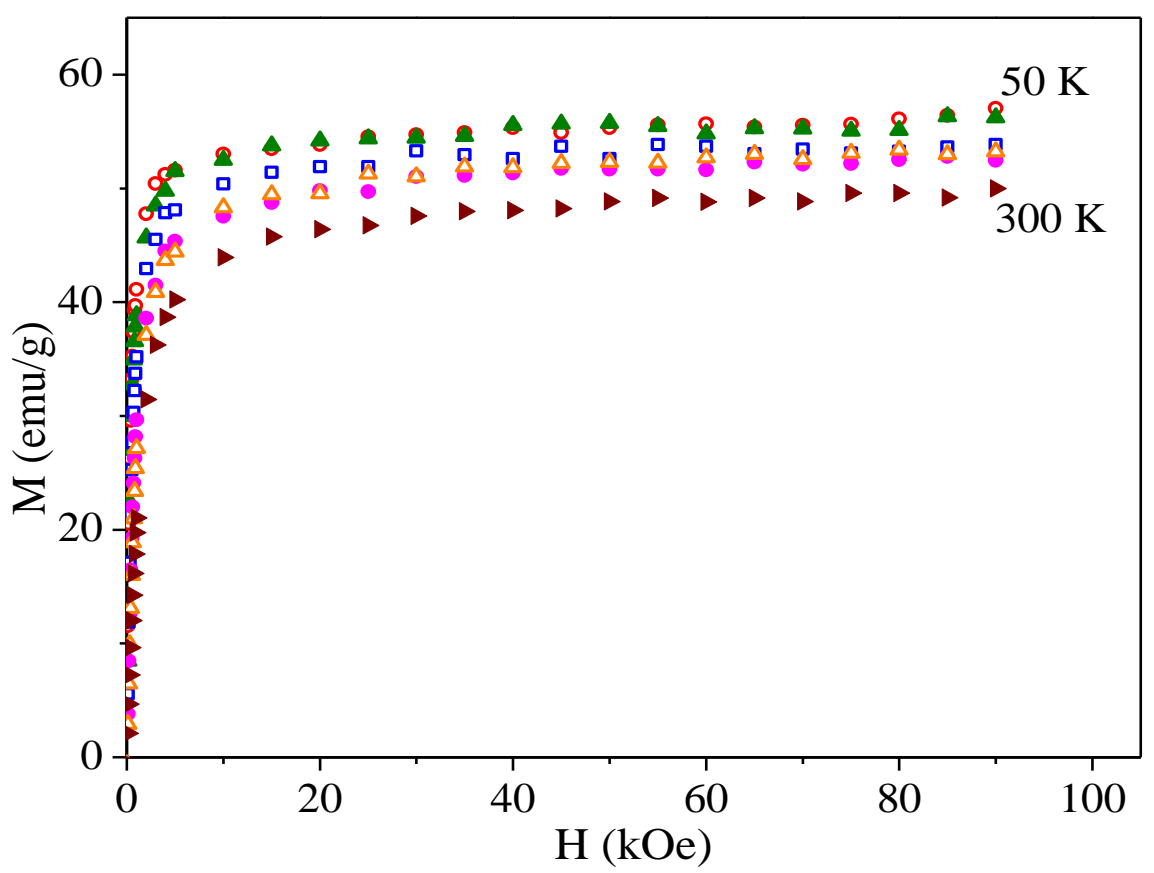

Figure 4.6: $M$ vs applied magnetic field for $\mathrm{T}=50 \mathrm{~K}$ (open red circles), $100 \mathrm{~K}$ (solid green triangles), $150 \mathrm{~K}$ (open blue circles), $200 \mathrm{~K}$ (solid pink circles), $250 \mathrm{~K}$ (open orange triangles) and $300 \mathrm{~K}$ (solid brown triangles).

\subsubsection{Temperature and Frequency Dependence of ac Susceptibilities}

Chapter 2 provided a brief introduction to the ac magnetic susceptibilies $\chi^{\prime}=M^{\prime} / H_{o}$, the in-phase ac magnetic susceptibility, and $\chi^{\prime \prime}=M^{\prime \prime} / H_{o}$, the out-of-phase ac magnetic susceptibility. For the $7.0 \mathrm{~nm}$ $\gamma-\mathrm{Fe}_{2} \mathrm{O}_{3}$ sample, the temperature dependence of these susceptibilities was measured for $f_{m}$ from $10 \mathrm{~Hz}$ to $10 \mathrm{kHz}$ using $H_{o}=10$ Oe as the amplitude of the ac magnetic field. The results are shown in Fig. 4.7 where the $10 \mathrm{kHz}$ data has been left out for the $\chi^{\prime \prime}$ case due to excessive noise. The already low signal for $\chi^{\prime \prime}$ was obscured too much to perform reliable analysis with the additional noise that was repeatedly seen when using the maximum frequency achievable with the ACMS. Attempts to reduce the ac magnetic field amplitude did not improve the quality of the $10 \mathrm{kHz}$ data. For future work, additional frequencies of $20 \mathrm{~Hz}, 200 \mathrm{~Hz}$, and $2 \mathrm{kHz}$ were added to make up for removing $10 \mathrm{kHz}$ from the measurement. 


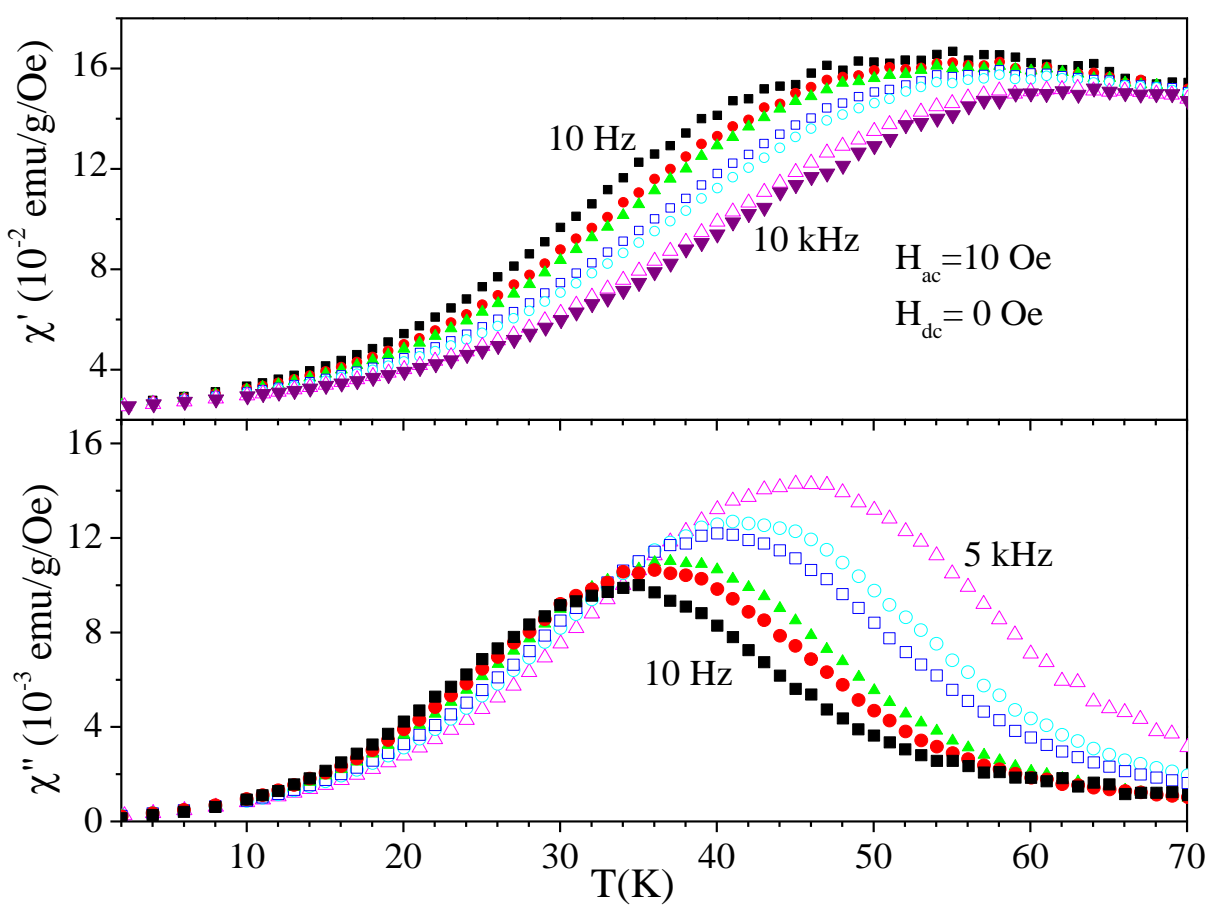

Figure 4.7: The in-phase (upper) and out-of-phase (lower) ac susceptibility data as a function of temperature for $f_{m}=10 \mathrm{~Hz}, 50 \mathrm{~Hz}, 100 \mathrm{~Hz}, 500 \mathrm{~Hz}, 1 \mathrm{kHz}, 5 \mathrm{kHz}$, and $10 \mathrm{kHz}$ (10 kHz not pictured for out-of-phase data) with an ac field amplitude of 10 Oe.

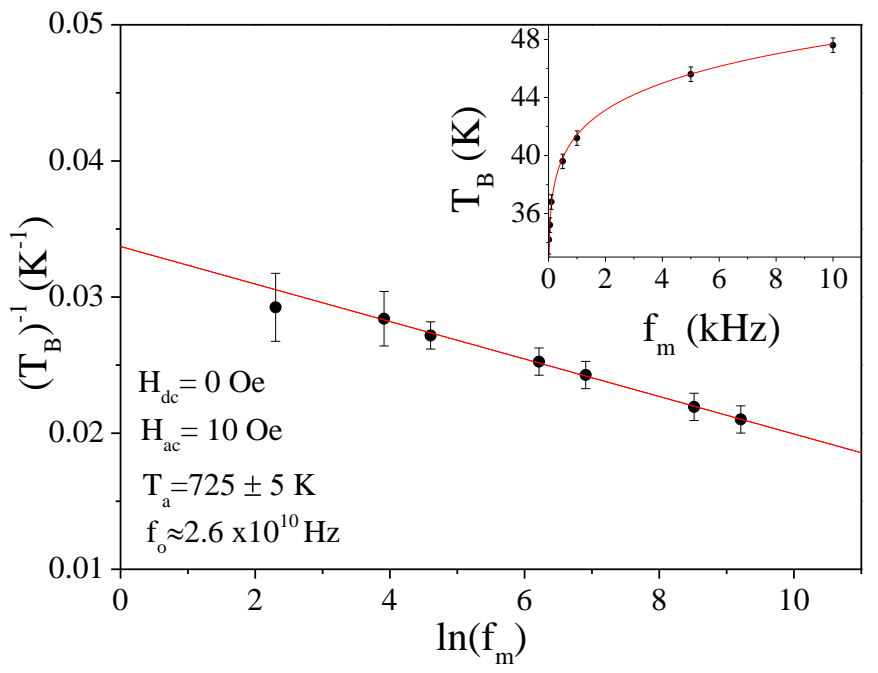

Figure 4.8: Inverse of the blocking temperature determined form the $\chi^{\prime \prime}$ data of Fig. 4.6 vs. natural log of the applied field frequency. Red line is fit to Eq. 4.6. Inset: Blocking temperature determined from the $\chi^{\prime \prime}$ data of Fig. 4.7 vs. applied magnetic field frequency fit to Eq. 4.3 (red curve). 
For convenience in the discussion, the following equation from Chapter 2 for the blocking temperature is reproduced:

$$
T_{B}=T_{o}+\frac{K_{a} V}{k_{B} \ln \left(f_{o} / f_{m}\right)}
$$

with $T_{o}$ representing the strength of the interparticle interaction and $T_{a}=K_{a} V / k_{B}$ the effective temperature related to the energy barrier.

\subsection{Data Analysis and Interpretation}

\subsubsection{Néel-Brown Relaxation and Interparticle Interaction}

In general, dc magnetic measurements alone do not allow for the accurate determination of the effects of interparticle interactions. For this reason, values of $f_{o}$ and $T_{a}$ determined from dc data can be misleading when $T_{o}=0 \mathrm{~K}$ is used in Eq. 4.2 when applied to interacting nanoparticles. As noted in Chapter 2, $T_{B}$ is determined from the peak in the $\chi^{\prime \prime}$ vs. $T$ data of Fig. 4.7 and the results are plotted against $f_{m}$ in the inset of Fig. 4.8. Using the values of $T_{B}$ determined for each $f_{m}, \Phi=0.12 \pm 0.01$ is calculated which is close to the often-used cutoff of 0.13 for non-interacting particles. From this, it is determined that the effects of any interparticle interactions are small in this sample so $T_{o}$ will be small compared to the observed $T_{B}$.

A direct fit of the data in the inset of Fig. 4.8 to Eq. 4.2 was not performed because the small number of data points (7) and three variables that were allowed to vary could produce non-unique solutions. To analyze this data, Eq. 4.2 was rewritten as

$$
\left(T_{B}-T_{o}\right)^{-1}=-\frac{\ln \left(f_{m}\right)}{T_{a}}+\frac{\ln \left(f_{o}\right)}{T_{a}}
$$

which allows plotting the results against $\ln \left(f_{m}\right)$ to obtain a line and quickly analyze the data to determine the values of $f_{o}$ and $T_{a}$ that give the best fit for any given value of $T_{o}$. Values of $T_{o}$ were selected and the resulting fits analyzed to determine that $T_{o}=0 \mathrm{~K}$ produced the most linear plot with the highest $R^{2}$ value. This $T_{o}$ was in agreement with the magnitude of $\Phi=0.12 \pm 0.01$ indicating that interactions between the particles are negligible, most likely due to the oleic acid coating present on the particle surfaces. From the fit to Eq. 4.3 shown in Fig. 4.8 , the values of $T_{a}=725 \pm 5 \mathrm{~K}$ and $f_{o}=2.6 \times 10^{10} \mathrm{~Hz}$ were determined. This value of $f_{o}$ is near the value expected for ferromagnetic nanoparticles without interparticle interactions based on other published work (Singh, et al., 2009) and is held constant for all of the $\gamma-\mathrm{Fe}_{2} \mathrm{O}_{3}$ nanoparticles discussed in this dissertation. The value of $T_{a}$ determined is used to estimate the anisotropy constant $K_{a} \cong 5.5 \times 10^{5} \mathrm{erg} / \mathrm{cm}^{3}$ — nearly twelve times the bulk value of $4.7 \times 10^{4} \mathrm{erg} / \mathrm{cm}^{3}$ reported in literature (Birks, 1950). 


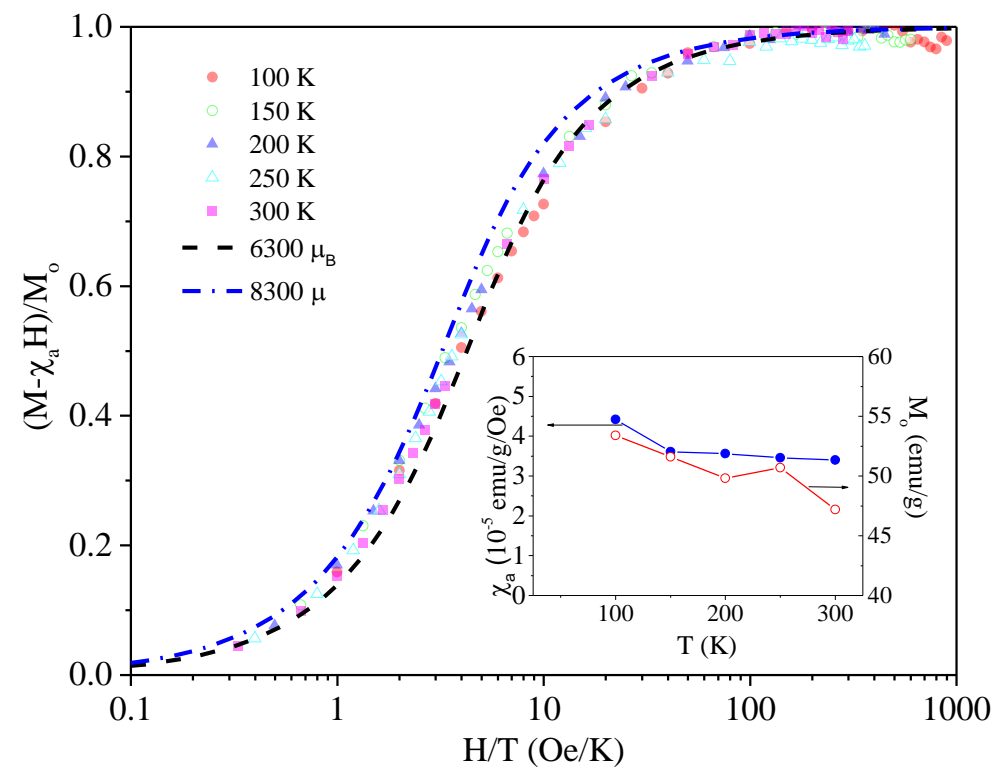

Figure 4.9: Fit of the M vs. $H$ data of Fig. 4.6 at T $=100 \mathrm{~K}, 150 \mathrm{~K}, 200 \mathrm{~K}, 250 \mathrm{~K}$ and $300 \mathrm{~K}$ to the modified Langevin function of Eq. 2.37 with the determined parameters listed in Table 4.1. The two solid curves represent the simulated fits to Eq. 4.1 with $\mu_{p}=6300 \mu_{B}$ and $8300 \mu_{B}$. The inset shows the temperature dependence of $M_{o}$ and $\chi_{a}$ determined from the fit with the lines connecting the data points shown for visual clarity.

\subsubsection{Analysis of $M$ vs. $H$ above $T_{B}$}

The analysis of the $M$ vs. $H$ data is considered next. The data in Fig. 4.6 was fit to Eq. 4.1 and the results are given in Table 4.1. The temperature dependent saturation magnetization $M_{o}$ and the linear component of the susceptibility $\chi_{a}$ both decrease with increasing temperature (Inset Fig. 4.9). In this model, the magnetic moment per particle was expected to remain relatively constant with temperature. To that end, the data in Fig. 4.6 were plotted as $\left(M-\chi_{a} H\right) / M_{o}$ vs. $H / T$ so the points fall onto a single curve (Fig. 4.9). While the data in Fig. 4.9 do seem to converge, the average magnetic moment per particle, $\mu_{p}=7300 \pm 500 \mu_{B}$ appears to increase slightly with decreasing temperature. The overall quality of the fit to the Eq. 4.1indicates that the nanoparticles are exhibiting superparamagnetic behavior above $T_{B}$.

\subsubsection{Analysis of $M$ vs. $T$ above $T_{B}$}

The data of $M$ vs. $T$ shown in Fig. 4.3 are considered next. Recall that the magnetization of superparamagnetic nanoparticles is given by Eq. 4.1. In the high-temperature limit, where $\mu_{p} H / k_{B} T \ll 1$, Eq. 4.1 can be simplified to (Seehra \& Punnoose, 2001)

$$
M=\left(\frac{M_{o} \mu_{p}}{3 k_{B} T}+\chi_{a}\right) H
$$


Experimental observations have shown that $M_{o}$ decreases linearly with $T$ near $T_{N}$ (Makhlouf, et al., 1997). The temperature dependent saturation magnetization can be written as (Seehra \& Punnoose, 2001)

$$
M_{o}=M^{*}\left(T_{N}-T\right) / T_{N}
$$

where $M^{*}$ is the saturation magnetization extrapolated to $T=0 \mathrm{~K}$ and $T_{N}$ is the Néel temperature. Substituting Eq. 4.5 into Eq. 4.4 gives

$$
M=\left(\frac{C}{T}+\chi_{o}\right) H
$$

where

$$
C=\frac{\mu_{p} M^{*}}{3 k_{B}}
$$

so that the susceptibility can be written as

$$
\chi=M / H=\chi_{o}+\frac{C}{T}
$$

with

$$
\chi_{o}=\chi_{a}-C / T_{N}
$$

Extrapolating the $M_{o}$ data given in Table 4.1 to $T=0 \mathrm{~K}$ gives $M^{*} \approx 65 \mathrm{emu} / \mathrm{g}$. This value was then checked using the relationship

$$
\mu_{p}=M^{*} \rho V
$$

using $\mu_{p} \cong 7300 \mu_{B}$ determined in Section 4.4.2, $V=\pi D^{3} / 6$ for spherical particles, and $\rho=4.856 \mathrm{~g} / \mathrm{cm}^{3}$ for the density of $\gamma-\mathrm{Fe}_{2} \mathrm{O}_{3}$. Solving Eq. 4.10 for $D$ gives an average particle diameter $D=7.5 \mathrm{~nm}$ in reasonable agreement with the value of $\langle D\rangle$ determined from the TEM data in Section 3.2.

The data of $M$ vs. $T$ in Fig. 4.3 is divided by 100 Oe to yield $\chi$ and $\chi^{-1}$ is plotted for the ZFC and FC cases in Fig. 4.10. It is evident from the plot that $\chi^{-1}$ vs. $T$ is not linear and so Eq. 2.13 describing the Curie law variation of a paramagnet does not describe the temperature dependence of $\chi$ for a superparamagnet. This is also evident from Eq. 4.8 because of the additional term $\chi_{o}$. To test the fit to Eq. 4.8 requires first determining $\chi_{o}$. To determine $\chi_{o}, \chi$ vs. $1 / T$ was plotted and a line was fit to only the highest temperature data available $(300 \mathrm{~K}-350 \mathrm{~K})$ where the contribution from $C / T$ is the most negligible. From the intercept of this line, $\chi_{o}=0.0186 \pm 0.0005 \mathrm{emu} / \mathrm{g}$ Oe was determined. Then, a plot of $\left(\chi-\chi_{o}\right)^{-1}$ vs. $T$ was obtained that did show linear behavior for $T$ significantly above $T_{B}$ (Fig. 4.10) as expected from Eq. 4.8. 


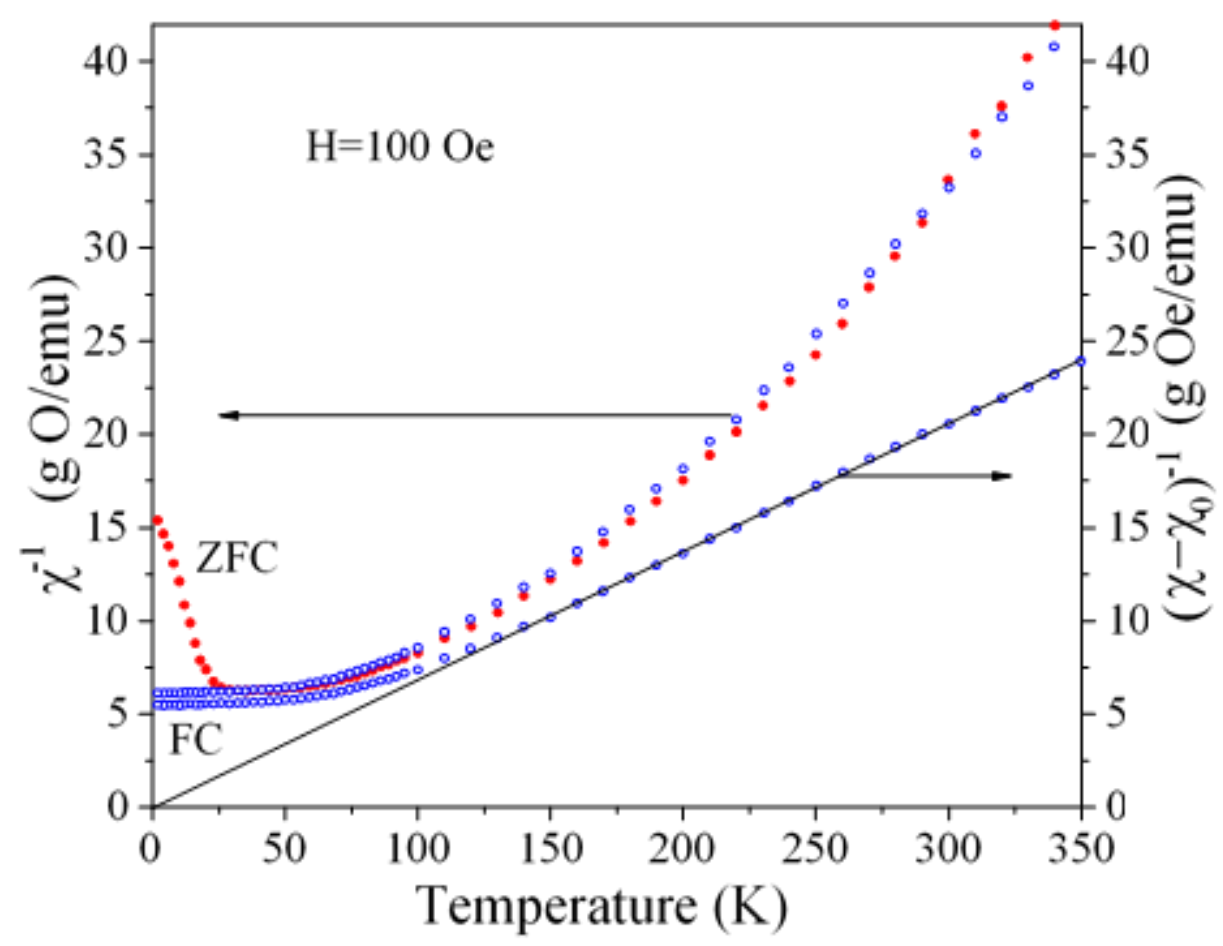

Figure 4.10: Using the data of Fig. 4.3, the plots of $\chi^{-1}$ vs $T$ (left axis) and $\left(\chi-\chi_{o}\right)^{-1} v s . T$ (right axis) are shown. The solid line is a fit to the high-temperature $\left(\chi-\chi_{o}\right)^{-1}$ data.

The high temperature $\left(\chi-\chi_{o}\right)^{-1}$ vs. $T$ data of Fig. 4.10 was then fit to a line and the slope taken as $1 / C$. The value of $C=14.6 \pm 0.1 \mathrm{emu} \mathrm{K} / \mathrm{g}$ Oe determined was then used to find the value of $\mu_{p}=10027 \pm 140 \mu_{B}$ from Eq. 4.7. This value is much larger than the value determined from the fit to the modified Langevin given by Eq. 4.1. To understand the reason for this discrepancy, one must consider the limits taken to arrive at Eq. 4.8.

The high temperature limit used to arrive at Eq. 4.8 is analogous to the low-field limit, that is, $H / T \ll 1$. In the low-field limit, the contributions to the magnetization are dominated by the largest particles in the size distribution (Ibrahim, et al., 1992); therefore, the magnetic moment determined from Eq. 4.8 would be larger than the magnetic moment determined from the fit to Eq. 4.1 which should include contributions from all particles. When the particle diameter is estimated from Eq. 4.10 using $\mu_{p}=10027 \mu_{B}, D=8.3 \mathrm{~nm}$ is found, a value significantly larger than the average value as expected from the above argument.

\subsubsection{Analysis using a Distribution of Magnetic Moments}

In section 4.4.3, it was shown that the low-field magnetic response was dominated by the largest particles in the sample. In fact, it is reasonable to expect that a distribution of particle sizes results in a distribution in the magnitudes of $\mu_{p}$ given the linear relationship between $\mu_{p}$ and $V$ shown in Eq. 4.10. 
This issue of a distribution of magnetic moments has been addressed by Ibrahim, et al. (1992) using the analysis of Richardson \& Desai (1976) who found that most catalysts are described by a lognormal distribution in particle sizes. Based on the direct relationship in Eq. 4.10, it is reasonable to assume that if the volume is lognormally distributed, then the magnetic moments are lognormally distributed. This issue was addressed by Silva et al. (2006) in connection with the magnetic moment distribution in ferrihydrite nanoparticles.

The probability distribution function for a lognormal distribution of magnetic moments, $\mu$, is given by

$$
f(\mu)=\frac{1}{\mu s \sqrt{2 \pi}} \exp \left\{\frac{-\left[\ln \left(\mu / \mu_{o}\right)\right]^{2}}{2 s^{2}}\right\}
$$

where $\mu_{o}$ is the median value of $\mu$, which is to say $\mu_{\mathrm{o}}$ divides the distribution function so that the area under the curve defined by $\mathrm{f}(\mu)$ from 0 to $\mu_{\mathrm{o}}$ comprises $1 / 2$ the total area under $f(\mu)$. The value $s$ in Eq. 4.11 represents the width of the distribution. In the case of a normal distribution, $\mu_{o}$ would represent the center of the distribution curve whereas its relationship to the center of the distribution curve or the mean in a log normal distribution can vary greatly depending on the shape of the distribution curve. For a group of particles with a large variation in magnetic moments, the value of $\mu_{o}$ may be drastically different than the average magnetic moment and also distinctly different from the most probable particle magnetic moment.

The average moment in a log normal distribution is given by

$$
\langle\mu\rangle=\mu_{o} \exp \left[\frac{s^{2}}{2}\right]
$$

therefore, for samples with narrow distributions (small s values), $\langle\mu\rangle$ will be near but slightly higher than the value of $\mu_{o}$, while for broad distributions (larger s values) $\langle\mu\rangle$ can be much larger than $\mu_{o}$. This expression for $\langle\mu\rangle$ comes from

$$
\int_{0}^{\infty} \mu f(\mu) d \mu=\frac{1}{s \sqrt{2 \pi}} \int_{0}^{\infty} \exp \left\{\frac{-\left[\ln \left(\mu / \mu_{o}\right)\right]}{2 s^{2}}\right\} d \mu
$$

which gives

$$
\langle\mu\rangle=\lim _{\mu \rightarrow \infty} \frac{\mu_{o}}{2} \exp \left(\frac{s^{2}}{2}\right) \operatorname{erf}\left[\frac{\ln \left(\mu / \mu_{o}\right)-s^{2}}{\sqrt{2} s}\right]-\lim _{\mu \rightarrow 0} \frac{\mu_{o}}{2} \exp \left(\frac{s^{2}}{2}\right) \operatorname{erf}\left[\frac{\ln \left(\mu / \mu_{o}\right)-s^{2}}{\sqrt{2} s}\right]
$$

where $\operatorname{erf}(\mathrm{x})$ is the error function. This expression can be simplified to get

$$
\langle\mu\rangle=\frac{\mu_{o}}{2} \exp \left(\frac{s^{2}}{2}\right) \lim _{x \rightarrow \infty}\{\operatorname{erf}[x]-\operatorname{erf}[-x]\}=\mu_{o} \exp \left(\frac{s^{2}}{2}\right) .
$$


The peak in the distribution function given in Eq. 4.11 is located at $\mu_{\text {peak }}$ given by

$$
\mu_{\text {peak }}=\mu_{o} \exp \left(-s^{2}\right)
$$

The peak will shift towards smaller values of $\mu$ for increasing values of $s$ and will approach $\mu_{o}$ as $s$ goes to 0 and the probability distribution becomes more symmetric. The parameter $s$ is related to the mean and standard deviation of the distribution function by the equation

$$
s=\sqrt{\ln \left(1+\frac{\sigma^{2}}{\langle\mu\rangle^{2}}\right)}
$$

which gives

$$
\sigma=\langle\mu\rangle\left[\exp \left(s^{2}\right)-1\right]^{1 / 2}
$$

The standard deviation will increase with increasing $s$ and the distribution function will approach a delta function for $s$ approaching 0 . While the latter cannot be shown analytically since $f(\mu)$ is infinitely differentiable, this is determined as the area under the curve described by $f(\mu)$ remains 1 for any combination of $\mu_{o}$ and $s$ including the case when $s$ approaches 0 and the peak width becomes infinitely small.

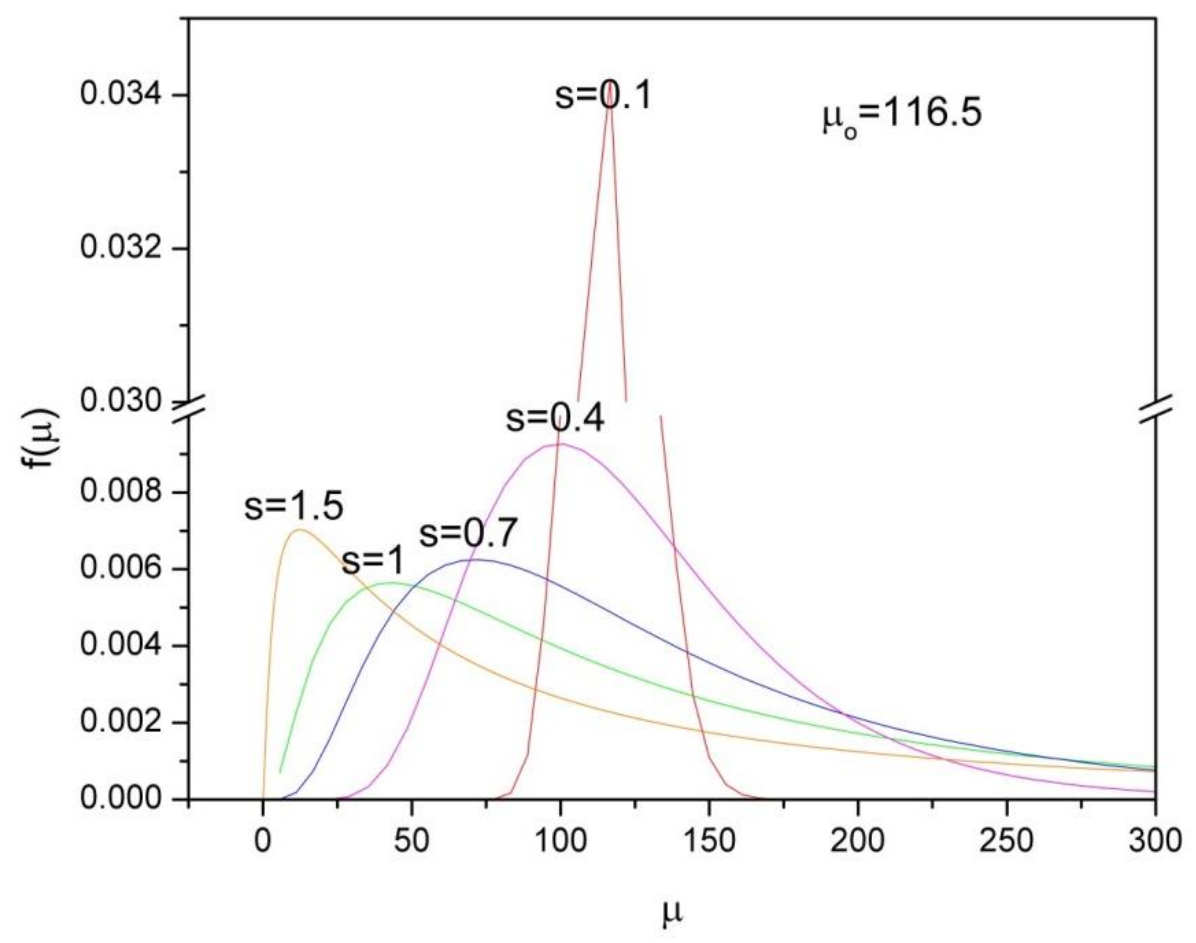

Figure 4.11: Simulated distribution functions for $\mu_{o}=116.5 \mu_{B}$ with $s=0.1,0.4,0.7,1.0$, and 1.5. 
Table 4.2: Simulated values of mean magnetic moment $\langle\mu\rangle$, standard deviation $\sigma$, and location of the peak in $f(\mu)$ of Fig. 4.11 for $\mu_{o}=116.5 \mu_{B}$ and the calculated variability $\sigma /\langle\mu\rangle$.

\begin{tabular}{|c|c|c|c|c|}
\hline$s$ & $\langle\mu\rangle\left(\mu_{\mathrm{B}}\right)$ & $\sigma\left(\mu_{\mathrm{B}}\right)$ & $\mu_{\text {peak }}\left(\mu_{\mathrm{B}}\right)$ & $\sigma /\langle\mu\rangle$ \\
\hline 0.1 & 117.1 & 11.7 & 115.3 & 0.102 \\
\hline 0.4 & 126.2 & 52.6 & 99.3 & 0.417 \\
\hline 0.7 & 148.8 & 118.3 & 71.4 & 0.795 \\
\hline 1.0 & 192.1 & 251.8 & 42.9 & 1.31 \\
\hline 1.5 & 358.8 & 1045.5 & 12.3 & 2.91 \\
\hline
\end{tabular}

In Fig. 4.11, the probability distribution function $f(\mu)$ has been simulated for $\mu_{o}=116.5 \mu_{B}$ as in Tiwari \& Rajeev (2012) for different values of $s$. From the simulated plots, the shift in $\mu_{\text {peak }}$ to smaller values of $\mu$ with increasing $s$ can be seen. It is also clear that, for $s<1$, decreasing $s$ corresponds with decreasing peak width as well as increased symmetry. Table 4.2 contains the calculated mean particle moment $\langle\mu\rangle$, peak location $\mu_{\text {peak }}$, and standard deviation $\sigma$ for the plotted values of $s$ as well as the variability $(\sigma /\langle\mu\rangle)$. There exists a value of $s$ where the variability becomes greater than one, due to the incredibly skewed nature of the distribution; that is, for $\left[\exp \left(s^{2}\right)-1\right]^{1 / 2}>1$ or $s \geq \sqrt{\ln (2)} \approx 0.83$, the value of $\sigma$ will exceed the value of $\langle\mu\rangle$. For $\sigma\rangle\langle\mu\rangle$ it is no longer appropriate to attempt to make comparisons between the quantities $\mu_{o},\langle\mu\rangle$, and the quantity $\mu_{p}$ obtained from a fit to Eq. 4.1.

Including the effect of the magnetic moment distribution in the modified Langevin fit to $M$ vs $H$ requires modifying Eq. 4.1 in which $M_{o}=N\langle\mu\rangle$ is the saturation magnetization for the temperature at which the measurement takes place and $N=1 / V$. The magnetization of an assembly of nanoparticles with a moment distribution function given by $f(\mu)$ is then

$$
M(H, T)=N \int_{0}^{\infty} \mu \mathcal{L}\left(\frac{\mu H}{k_{B} T}\right) f(\mu) d \mu+\chi_{a} H,
$$

where $N$ is the number density of the nanoparticle magnetic moments contributing to the total magnetization. Then, the data of Fig. 4.6 may be fit to Eq. 4.19 in an approach similar to that of Tiwari \& Rajeev (2012).

Fits to Eq. 4.19 can be difficult to obtain especially since the risk of over-parameterization is present. For effective fitting, it is helpful to first determine an approximate value for $M_{O}$ from the maximum $M$ value of the $M$ vs. $H$ plots and an approximate value for $N \approx\left(V \rho^{-1}\right)$, where $V$ is the volume of an average nanoparticle and $\rho$ is the density of the material. For example, the $7.0 \mathrm{~nm}$ sample should have a value for $N \sim 1.1 \times 10^{18} / \mathrm{g}$. The value of $N$ will be lower than this estimate and, depending on how tightly packed the sample and how thick the oleic acid coating, it may be easily an order of magnitude lower, but $10^{18}$ provides a safe place to start and a helpful upper limit. Using this estimate of $N$ along with the estimate of $M_{o}$, one can obtain a ballpark-figure for the range of $\langle\mu\rangle$ and begin the fitting 
Table 4.3: Parameters from the fit of Eq. 4.19 to the M vs. H data of Fig. 4.6.

\begin{tabular}{|c|c|c|c|c|c|c|c|}
\hline $\begin{array}{c}\mathrm{T} \\
\mathrm{K})\end{array}$ & $\begin{array}{c}\mathrm{N} \\
\left(10^{17} \mathrm{~g}^{-1}\right)\end{array}$ & $\begin{array}{c}\mu_{\mathrm{o}} \\
\left(\mu_{\mathrm{B}}\right)\end{array}$ & $\mathrm{s}$ & $\begin{array}{c}\langle\mu\rangle \\
\left(\mu_{\mathrm{B}}\right)\end{array}$ & $\begin{array}{c}\mathrm{M}_{\mathrm{s}}=\mathrm{N} *\langle\mu\rangle \\
(\mathrm{emu} / \mathrm{g})\end{array}$ & $\begin{array}{c}\chi_{\mathrm{a}}\left(10^{-5}\right. \\
\mathrm{emu} / \mathrm{g} \mathrm{Oe})\end{array}$ & $\mathrm{R}^{2}$ \\
\hline 150 & $8.37 \pm 0.10$ & $5958 \pm 5$ & $0.475 \pm 0.016$ & $6670 \pm 55$ & $51.8 \pm 1.1$ & $3.59 \pm 0.64$ & 0.9990 \\
\hline 200 & $8.37 \pm 0.20$ & $5750 \pm 216$ & $0.478 \pm 0.036$ & $6554 \pm 364$ & $50.9 \pm 4.1$ & $3.59 \pm 0.39$ & 0.9995 \\
\hline 250 & $8.36 \pm 0.08$ & $5846 \pm 5$ & $0.480 \pm 0.013$ & $6560 \pm 46$ & $50.9 \pm 0.8$ & $5.40 \pm 0.53$ & 0.9992 \\
\hline 300 & $8.35 \pm 0.07$ & $5463 \pm 5$ & $0.470 \pm 0.011$ & $6101 \pm 36$ & $47.2 \pm 0.7$ & $3.37 \pm 0.36$ & 0.9995 \\
\hline
\end{tabular}

by constraining the possible values of $N$ and $\mu_{o}$. The parameters determined from this fit are given in Table 4.3. A more detailed calculation for $N$ will be developed in Chapter 5.

\subsubsection{Temperature Dependence of Magnetization below $T_{B}$}

For the non-interacting particles, the differences between the $M$ vs. $T$ data for the zero fieldcooled and field-cooled conditions can be used to determine the distribution parameters of the blocking temperatures for the individual nanoparticles in the sample. Since the magnetization in the ZFC case is due only to the unblocked particles and the magnetization in the FC case is due to all particles (blocked and unblocked), the difference between the FC and ZFC cases $\Delta M=M_{F C}-M_{Z F C}$ is due only to the blocked particles and can be expressed as (Respaud, et al., 1998):

$$
\Delta M \cong \frac{A M_{s p}^{2} H}{3 K_{a}\left(T_{B}\right)} \int_{T}^{\infty} T_{B} f\left(T_{B}\right) d T_{B}
$$

where

$$
A=\ln \left(f_{o} / f_{m}\right)-1
$$

and $M_{s p}$ is the spontaneous magnetization; this is determined from the $2 \mathrm{~K}$ field-cooled magnetization. The parameters allowed to vary were $K_{a}, T_{B 0}$, and $\lambda_{B}$. The measurement field, $H=100$ Oe was held constant. Fitting to the data of Fig. 4.11 with Eq. 4.20 yields the following results: $K_{a}=2.3 \times 10^{5}$ $\mathrm{erg} / \mathrm{cm}^{3}, T_{B 0}=14 \pm 0.4 \mathrm{~K}$, and $\lambda_{B}=0.37 \pm 0.02$. Using $K_{a}=2.3 \times 10^{5} \mathrm{erg} / \mathrm{cm}^{3}$ in the equation

$$
\langle\mathrm{V}\rangle=\frac{\ln \left(f_{o}\right) \mathrm{k}_{\mathrm{B}}\left\langle\mathrm{T}_{\mathrm{B}}\right\rangle}{\mathrm{K}_{\mathrm{a}}}=\frac{\pi}{6}\left\langle\mathrm{D}_{\mathrm{TB}}\right\rangle^{3} \exp \left[\lambda_{\mathrm{B}}{ }^{2} / 3\right],
$$

yields $\left\langle\mathrm{D}_{\mathrm{TB}}\right\rangle=7.01$ in excellent agreement with the sizes determined by XRD and TEM. 


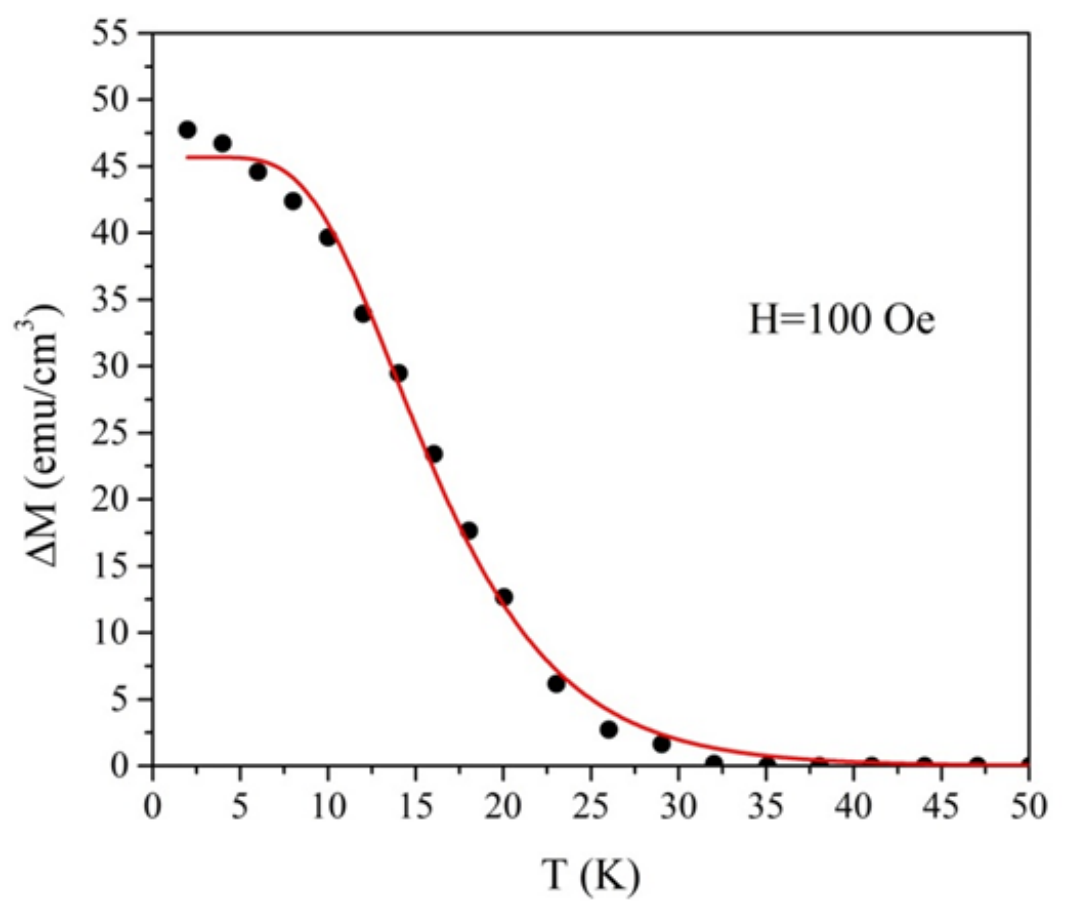

Figure 4.12: $\Delta M=M(F C)-M(X F C)$ data vs. $T$ with the fit to Eq. 4.20 shown as a solid red line and $K_{a}$ are not easily determined from this fitting, the values describing the distribution seem to be unique to the measured data and nearly independent of variations in the other parameters.

\subsection{Summary}

Magnetic measurements on $7.0 \mathrm{~nm} \gamma-\mathrm{Fe}_{2} \mathrm{O}_{3}$ nanoparticles and the results and analysis of these measurements have been presented with special attention given to the role of particle size distribution. From the analysis of the variation of $T_{B}$ with respect to $f_{m}$, the absence of significant interparticle interaction has been inferred. This result is attributed to the oleic acid coating present on the nanoparticles, similar to the observation in oleic acid-coated $\mathrm{NiO}$ nanoparticles compared to uncoated $\mathrm{NiO}$ nanoparticles (Shim, et al., 2008; Shim, et al., 2006). This analysis also yielded an anisotropy constant $K_{a}=5.5 \times 10^{5} \mathrm{erg} / \mathrm{cm}^{3}$ and the Néel-Brown attempt frequency of $f_{o}=2.6 \times 10^{10} \mathrm{~Hz}$ that will be used in Chapter 5 for the discussion of the size dependence of the magnetic properties of $\gamma-\mathrm{Fe}_{2} \mathrm{O}_{3}$ nanoparticles. The absence of any notable coercivity $H_{C}$ observed in this system is different than the large values reported in $\gamma-\mathrm{Fe}_{2} \mathrm{O}_{3}$ nanoparticle samples prepared by different methods (Millan, et al., 2007; Morales, et al., 1999; Parker, et al., 1993). Thus, the synthesis route may influence the magnetic properties of $\gamma-\mathrm{Fe}_{2} \mathrm{O}_{3}$ nanoparticles. Finally, it has been shown that the average magnetic moment per particle determined using a modified Langevin function that ignores a distribution in particle magnetic moments yields results consist with those obtained by considering a distribution of magnetic moments so long as the width parameter $s$ is significantly below 0.83 . For values of $s>0.83$, it has been shown that 
the fit to Eq. 4.1 is not advisable. The framework provided in this chapter forms the basis for the discussion of the magnetic properties of $\gamma-\mathrm{Fe}_{2} \mathrm{O}_{3}$ nanoparticles of different sizes in Chapter 5. 


\section{Chapter 5}

\section{Size Dependence of the Magnetic Properties of Maghemite Nanoparticles}

\subsection{Introduction}

In this chapter, the size dependence of the magnetic properties of oleic acid coated $\gamma-\mathrm{Fe}_{2} \mathrm{O}_{3}$ nanoparticles is presented and discussed. Magnetic properties of the $\gamma-\mathrm{Fe}_{2} \mathrm{O}_{3}$ samples with average diameters of $2.5 \mathrm{~nm}, 3.4 \mathrm{~nm}$, and $6.3 \mathrm{~nm}$ have been analyzed following the methods of Chapter 4 where the properties of the $7.0 \mathrm{~nm}$ particles have been presented. The blocking temperature $T_{B}$, Vogel-Fulcher temperature $T_{o}$, mean magnetic moment per particle $\langle\mu\rangle$, effective anisotropy constant $K_{a}$, and Curie constant $C$ of each sample are determined and compared with those for the $7.0 \mathrm{~nm}$ sample. In addition, the size dependence of the effective anisotropy values is investigated by including data from literature on other sizes of $\gamma-\mathrm{Fe}_{2} \mathrm{O}_{3}$ nanoparticles reported by other researchers. Other notable results included here are the derivation of Eq. 5.9 describing the relationship between $T_{o}$ and $\Phi$ as well as the experimental verification of this relationship. The importance of the distribution of particle diameters on the measured magnetic properties of $7.0 \mathrm{~nm} \gamma-\mathrm{Fe}_{2} \mathrm{O}_{3}$ particles has been discussed Chapter 4 and in the recent published paper by the author (Pisane, et al., 2015). Similar analysis on the other three particles sizes of $\gamma-\mathrm{Fe}_{2} \mathrm{O}_{3}$ is presented here along with the size dependence of the magnetic properties.

\subsection{Experimental Results}

\subsubsection{Temperature Dependence of the Magnetization of $\gamma-\mathrm{Fe}_{2} \mathrm{O}_{3}$ Nanoparticles}

The temperature-dependent dc magnetization $(M)$ was measured following the procedures of Chapter 4 for each of the three nanoparticle sizes of $2.5 \mathrm{~nm}, 3.4 \mathrm{~nm}$, and $6.3 \mathrm{~nm}$. The measurements were carried out under the ZFC and FC conditions as done for the $7.0 \mathrm{~nm}$ nanoparticles in Chapter 4. In Fig. 5.1, the plots of the $M$ vs. $T$ data of each sample taken using a magnetic field $H=100$ Oe are shown. The locations of the peaks in the ZFC data, below which the data for the FC and ZFC data separate, were used to determine the blocking temperatures $T_{B}$ listed in Table 5.1. Analysis and interpretations of these results are presented later in this chapter.

Table 5.1: Observed blocking temperatures for samples determined from the data of Figure 5.1.

\begin{tabular}{|c|c|}
\hline$\langle D\rangle(\mathrm{nm})$ & $T_{B}(\mathrm{~K})$ \\
\hline $7.0 \pm 0.8$ & 35 \\
\hline $6.3 \pm 0.6$ & 42 \\
\hline $3.4 \pm 0.7$ & 21 \\
\hline $2.5 \pm 0.7$ & 29 \\
\hline
\end{tabular}




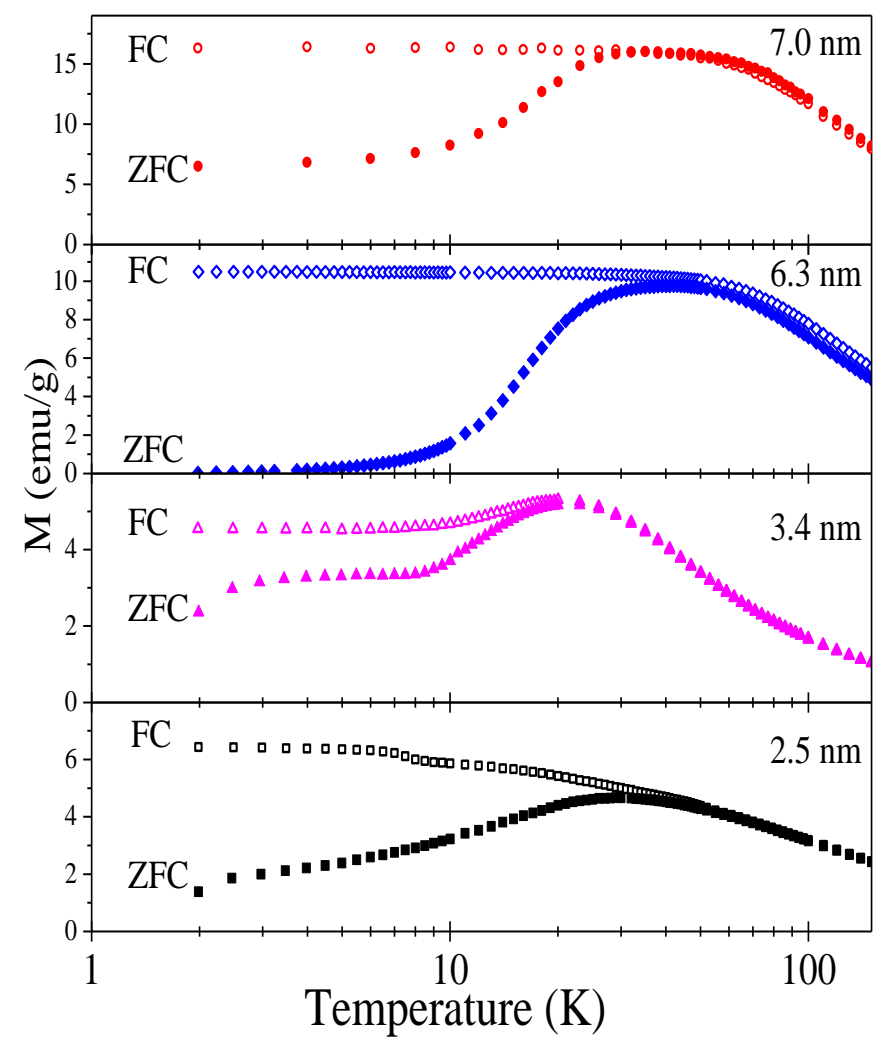

Figure 5.1: ZFC \& FC magnetization curves taken with a 100 Oe measuring/cooling field. Top to bottom: $7.0 \mathrm{~nm}, 6.3 \mathrm{~nm}, 3.4 \mathrm{~nm}$, and $2.5 \mathrm{~nm} \gamma-\mathrm{Fe}_{2} \mathrm{O}_{3}$ nanoparticle samples.

\subsubsection{Magnetic Field Dependence of dc Magnetization}

The blocking temperature $T_{B}$ separates the superparamagnetic region for $T>T_{B}$ from the blocked, magnetically frozen and partially ordered, region below $T_{B}$. Therefore, similar to the studies on the $7.0 \mathrm{~nm}$ particles described in Chapter 4, magnetic field dependence of the isothermal dc magnetization $M$ was investigated at select temperatures both above and below $T_{B}$ for each of the three samples. Figure 5.2 shows the data of $M$ vs. $H$ at $2 \mathrm{~K}$, well below $T_{B}$ of any of the samples, with the expanded view of the hysteresis loops for the low-field regions shown in the insets of Fig. 5.2. Except for the $6.3 \mathrm{~nm} \gamma-\mathrm{Fe}_{2} \mathrm{O}_{3}$ nanoparticles for which coercivity $H_{C}$ in excess of $400 \mathrm{Oe}$ is observed, the width of the hysteresis loops is nearly imperceptible in that $H_{C}$ for these samples is well within the experimental uncertainty of about 20 Oe for setting the field to zero. The temperature dependence of the coercivity $H_{C}$ and remanence $M_{R}$ measured from the hysteresis loops of the $6.3 \mathrm{~nm}$ sample is shown in Fig. 5.3. It is evident that both $H_{C}$ and $M_{R}$ approach zero as the temperature approaches $T_{B}$ from below. Such a temperature dependence of $H_{C}$ and $M_{R}$ appears to be a common feature that has been reported in other nanoparticle systems also such as in nanoparticles of $\beta-\mathrm{Ni}(\mathrm{OH})_{2}$ by Rall et al. (2010) and in flakes of ferrihydrite nanoparticles by Seehra 

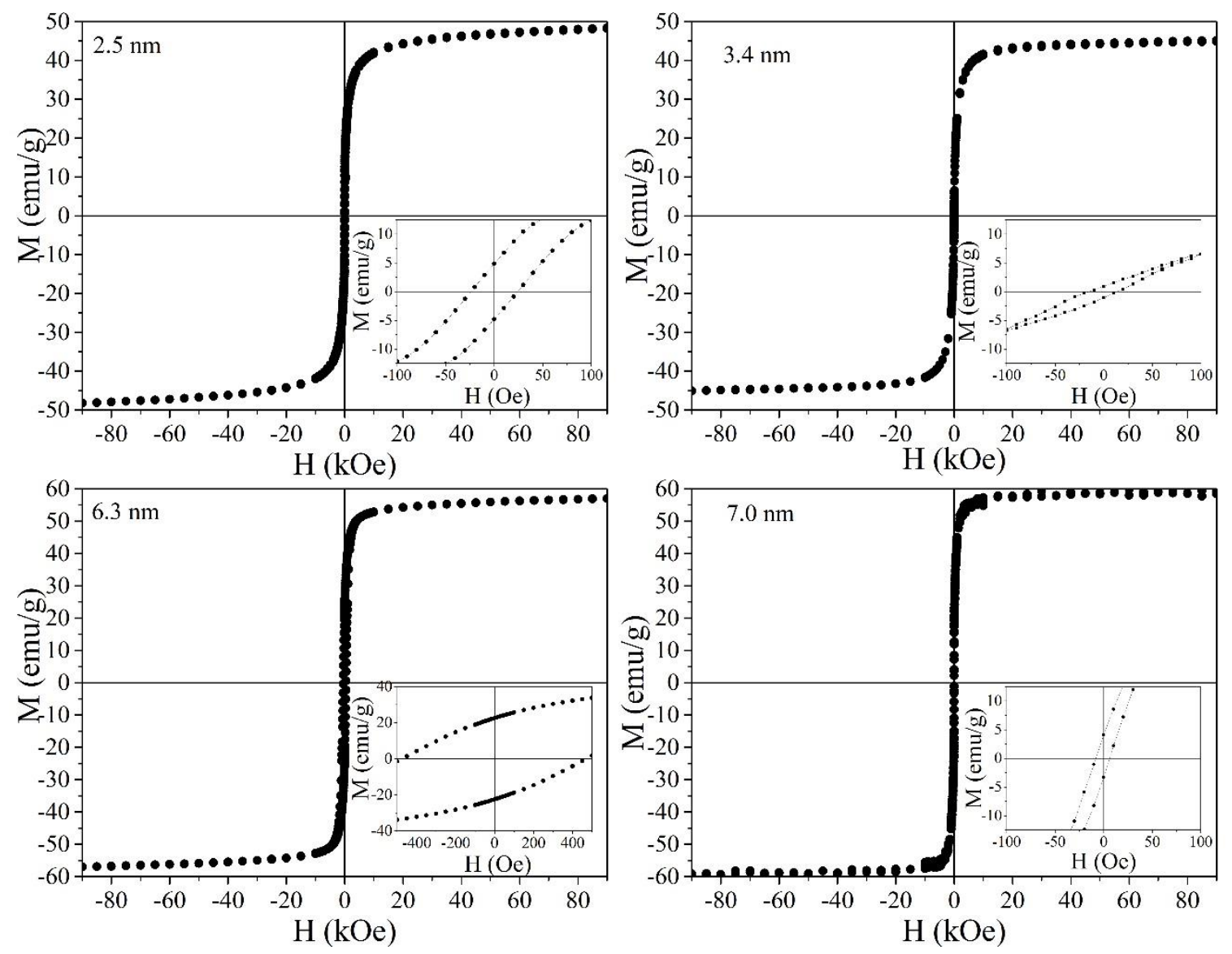

Figure 5.2: Hysteresis loops for all samples. Insets are zoomed in data near 0 Oe to show the hysteresis. Insets of each plot are the expanded low-field region showing the narrow width of the hysteresis loops for all but the $6.3 \mathrm{~nm}$ sample.

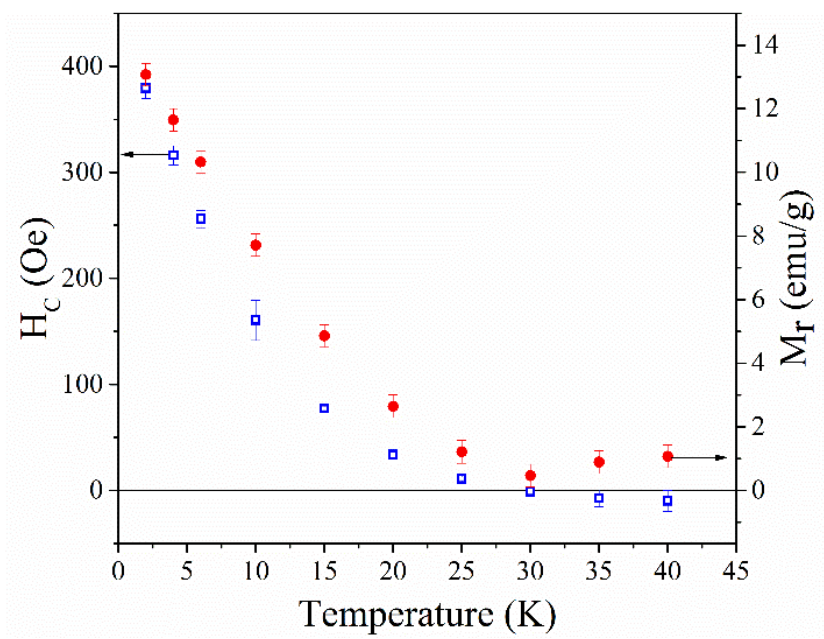

Figure 5.3: Temperature dependence of $H_{C}$ (open blue squares) and $M_{r}$ (solid red circles) for the 6.3 $n m \gamma-\mathrm{Fe}_{2} \mathrm{O}_{3}$ sample. Both $\mathrm{H}_{C}$ and $\mathrm{M}_{r}$ approach zero as the temperature approaches $T_{B}=42 \mathrm{~K}$. 
et al. (2010). Above $T_{B}$, the particles become unblocked and superparamagnetic leading to $H_{C}$ and $M_{R}$ becoming zero. Any residual non-zero magnitudes of $H_{C}$ and $M_{R}$ above average $T_{B}$ are likely due to a few remaining larger unblocked particles.

For the superparamagnetic region above $T_{B}, M$ vs. $H$ was also measured at select temperatures of $50 \mathrm{~K}, 100 \mathrm{~K}, 150 \mathrm{~K}, 200 \mathrm{~K}, 250 \mathrm{~K}, 300 \mathrm{~K}$, and $350 \mathrm{~K}$ (Fig. 5.4). Qualitatively, these curves are all similar in that at lower $H, M$ increases rapidly with increase in $H$ tending towards saturation at higher $H$. However, analysis of these plots presented later shows that only the plots of $M$ vs. $H$ above $150 \mathrm{~K}$ could be fitted satisfactorily in terms of modified Langevin function valid for the superparamagnetic region. One possible reason for this is that temperatures for $T<150$ are not far enough above $T_{B}$ for all sizes present in the distribution of the particles. Details of these procedures and results are given later.
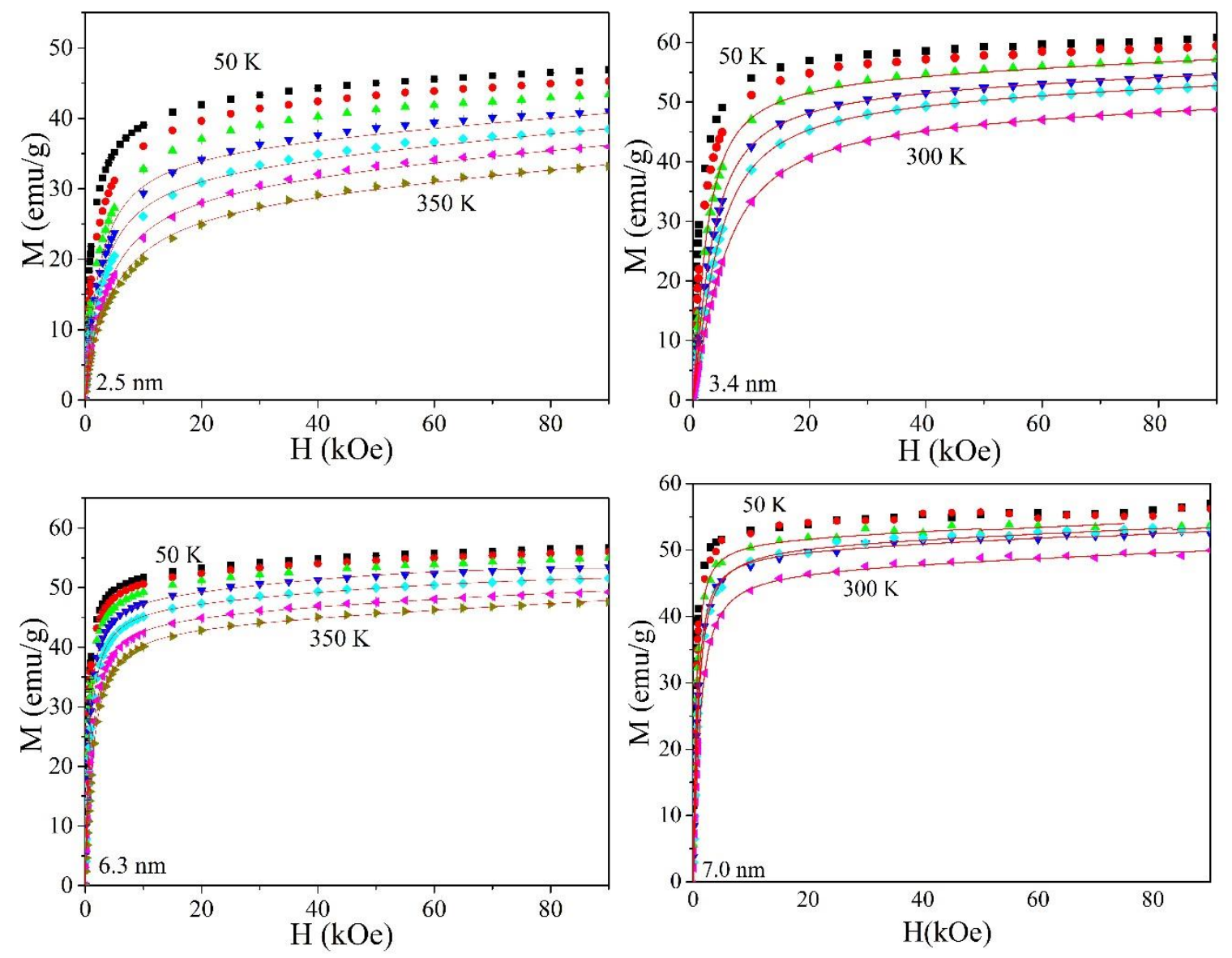

Figure 5.4: $M$ above $T_{B}$ measured for $H=0$ to $90 \mathrm{kOe}$ for all samples at $50 \mathrm{~K}$ intervals. Different color data points represent different temperatures black $=50 \mathrm{~K}$, red $=100 \mathrm{~K}$, green $=150 \mathrm{~K}$, blue $=200 \mathrm{~K}$, cyan $=250 \mathrm{~K}$, pink=300 K, and brown=350 K. Solid red curves are fits to a Modified Langevin equation with a lognormal distribution of magnetic moments given by Eq. 5.13.with the parameters given in Tables5.8, 5.9, 5.10, and 5.11. 


\subsubsection{Temperature and Frequency Dependence of ac Susceptibilities}

The temperature dependence of the ac susceptibilities, $\chi^{\prime}$ and $\chi^{\prime \prime}$, for the $7.0 \mathrm{~nm}$ sample presented in Chapter 4 was taken using an ac field amplitude $H_{a c}=10$ Oe at the frequencies $f_{m}$ of $10 \mathrm{~Hz}, 50 \mathrm{~Hz}$, $100 \mathrm{~Hz}, 500 \mathrm{~Hz}, 1 \mathrm{kHz}, 5 \mathrm{kHz}$, and $10 \mathrm{kHz}$. Due to the noise in the data for this sample, measurements for the $6.3 \mathrm{~nm}, 3.4 \mathrm{~nm}$, and $2.5 \mathrm{~nm}$ samples were performed using $H_{a c}=5$ Oe at frequencies $f_{m}=10 \mathrm{~Hz}$, $20 \mathrm{~Hz}, 50 \mathrm{~Hz}, 100 \mathrm{~Hz}, 200 \mathrm{~Hz}, 500 \mathrm{~Hz}, 1 \mathrm{kHz}, 2 \mathrm{kHz}$, and $5 \mathrm{kHz}$. The temperature dependence of $\chi^{\prime}$ and $\chi^{\prime \prime}$ for the four samples are shown in Figs. 5.5, 5.6, 5.7 and 5.8 for the samples with sizes of $7.0 \mathrm{~nm}, 6.3$ $\mathrm{nm}, 3.4 \mathrm{~nm}$ and $2.5 \mathrm{~nm}$, respectively. The blocking temperature $T_{B}$ for each frequency and each sample was determined from the position of the peak for $\chi^{\prime \prime}$. Analysis and interpretation of this data is presented in the following pages.

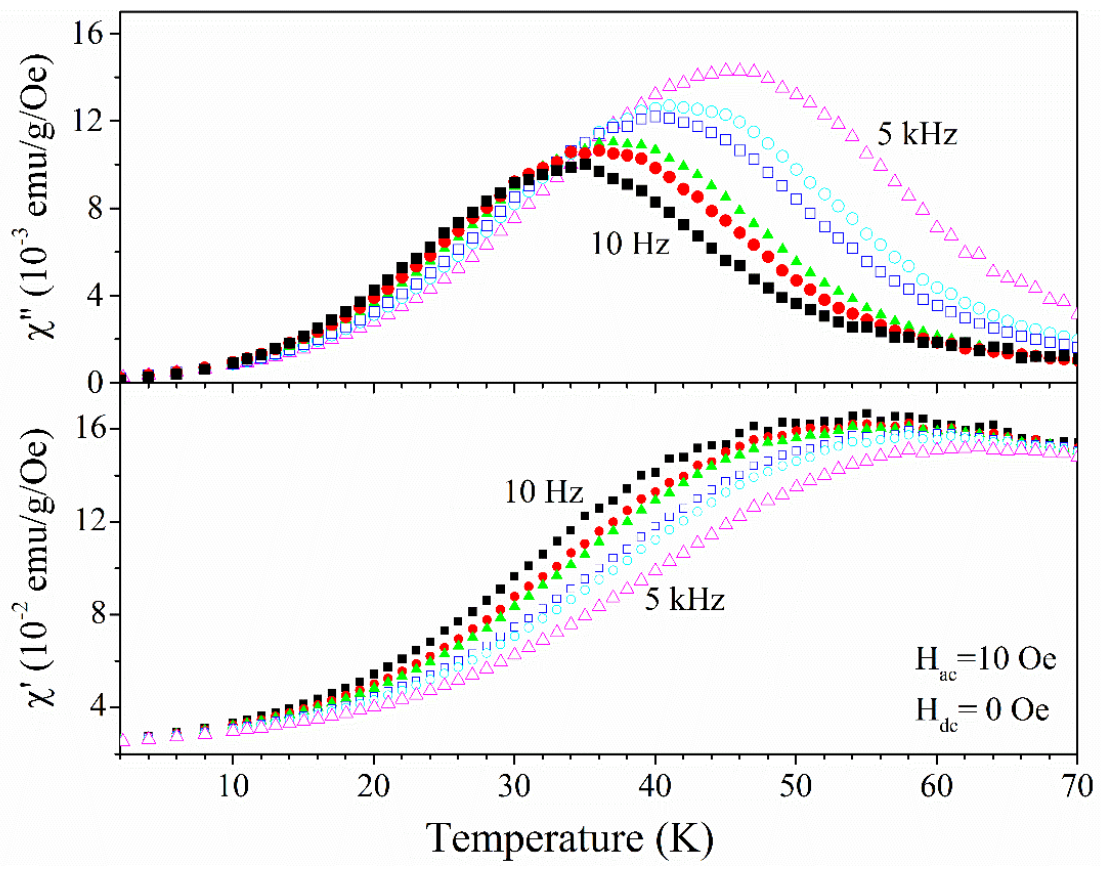

Figure 5.5: Out-of-phase (top) and in-phase (bottom) ac magnetic susceptibility of the $7.0 \mathrm{~nm} \gamma-\mathrm{Fe}_{2} \mathrm{O}_{3}$ nanoparticle sample taken with a 10 Oe ac magnetic field amplitude at $10 \mathrm{~Hz}, 50 \mathrm{~Hz}, 100 \mathrm{~Hz}, 500 \mathrm{~Hz}, 1$ $\mathrm{kHz}$, and $5 \mathrm{kHz}$. 


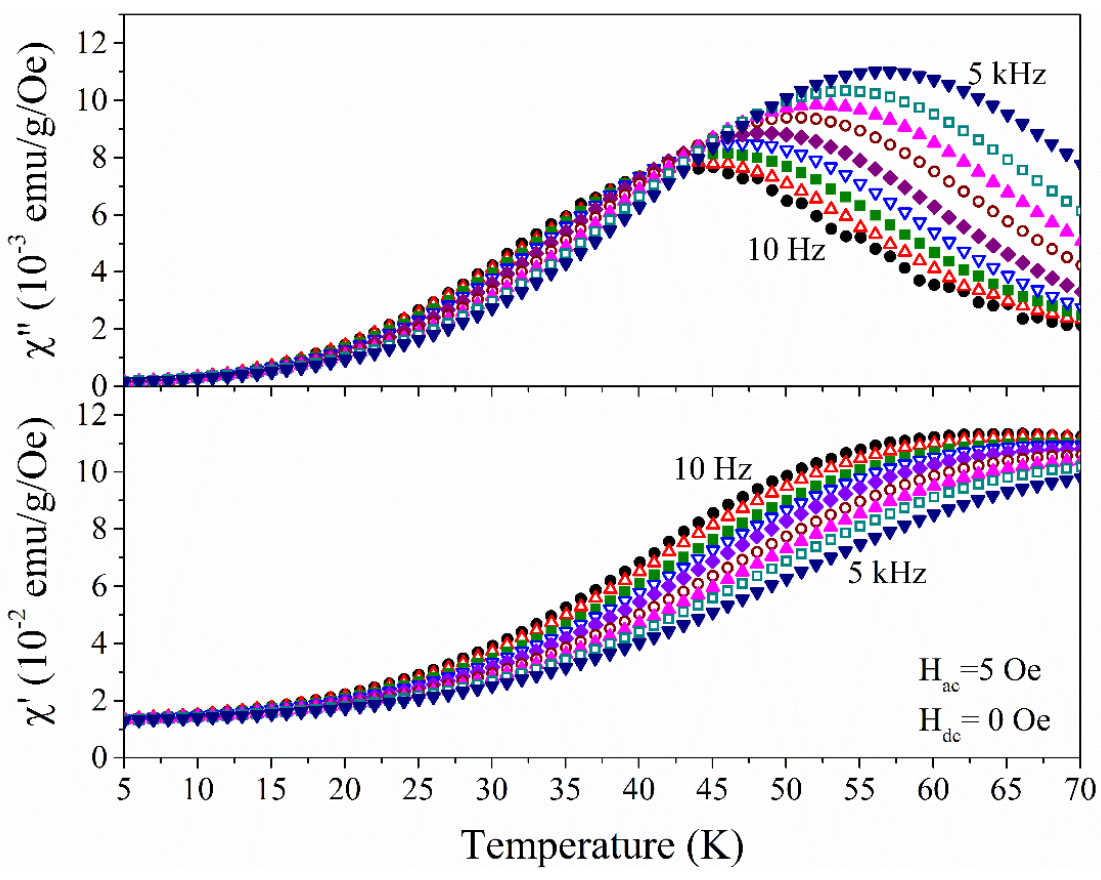

Figure 5.6: Out-of-phase (top) and in-phase (bottom) ac magnetic susceptibility of the $6.3 \mathrm{~nm} \gamma-\mathrm{Fe}_{2} \mathrm{O}_{3}$ nanoparticle sample taken with a 5 Oe ac magnetic field amplitude at $10 \mathrm{~Hz}, 20 \mathrm{~Hz}, 50 \mathrm{~Hz}, 100 \mathrm{~Hz}, 200$ $\mathrm{Hz}, 500 \mathrm{~Hz}, 1 \mathrm{kHz}, 2 \mathrm{kHz}$, and $5 \mathrm{kHz}$.

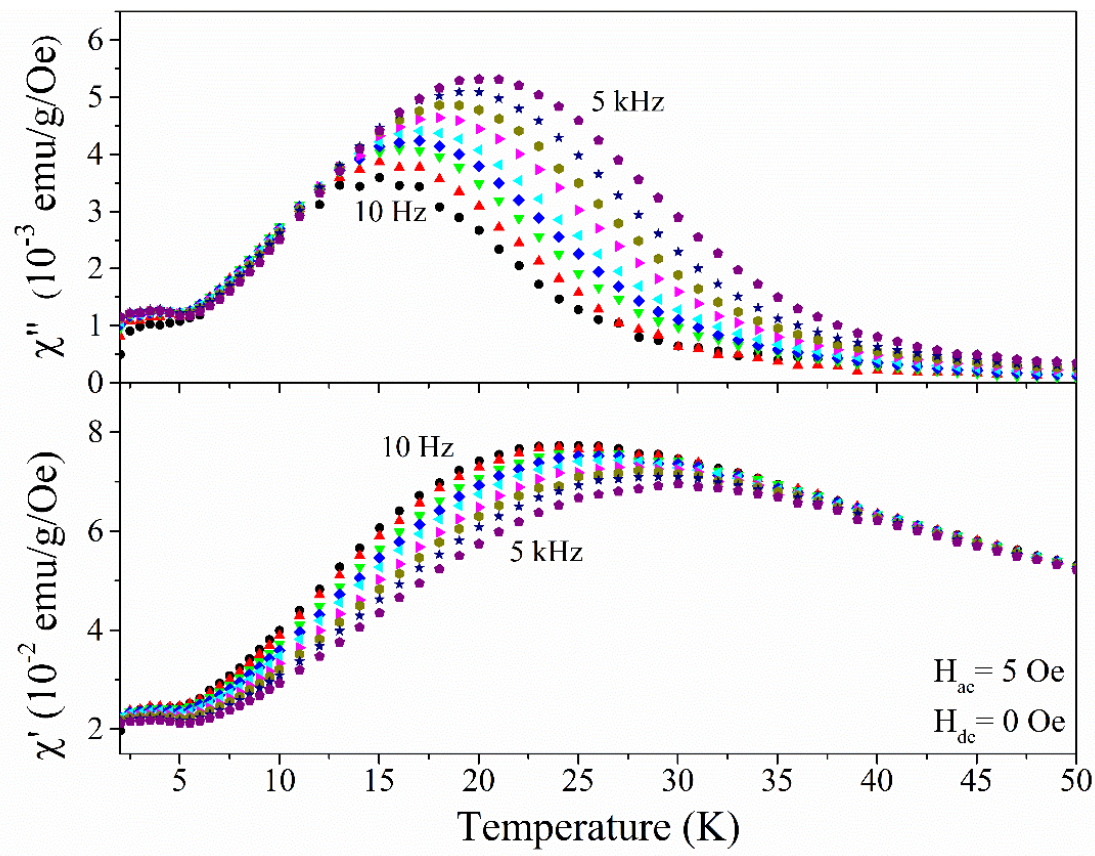

Figure 5.7: In-phase (bottom) and out-of-phase (top) ac magnetic susceptibility of the $3.4 \mathrm{~nm} \gamma-\mathrm{Fe}_{2} \mathrm{O}_{3}$ nanoparticle sample taken with a 5 Oe ac magnetic field amplitude at $10 \mathrm{~Hz}, 20 \mathrm{~Hz}, 50 \mathrm{~Hz}, 100 \mathrm{~Hz}, 200$ $\mathrm{Hz}, 500 \mathrm{~Hz}, 1 \mathrm{kHz}, 2 \mathrm{kHz}$, and $5 \mathrm{kHz}$. 


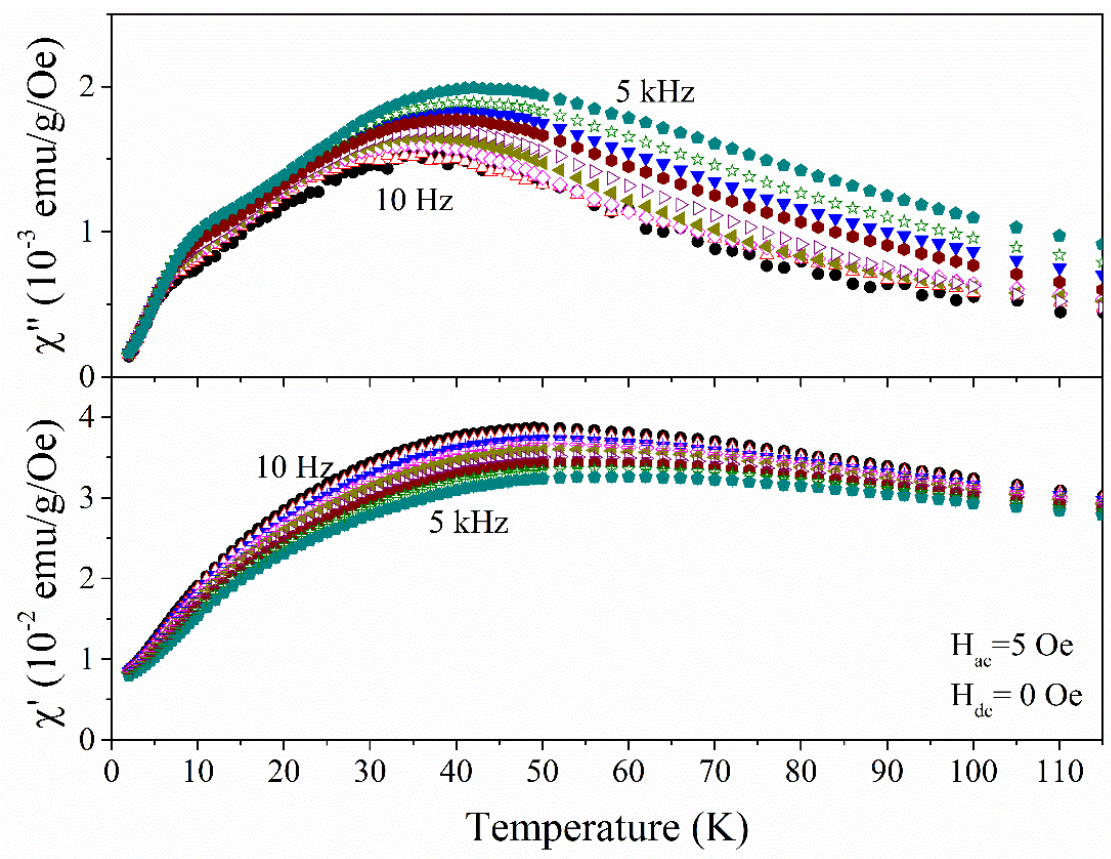

Figure 5.8: Out-of-phase (top) and in-phase (bottom) ac magnetic susceptibility of the $2.5 \mathrm{~nm} \gamma$-Fe2O3 nanoparticle sample taken with a 5 Oe ac magnetic field amplitude at $10 \mathrm{~Hz}, 20 \mathrm{~Hz}, 50 \mathrm{~Hz}, 100 \mathrm{~Hz}, 200$ $\mathrm{Hz}, 500 \mathrm{~Hz}, 1 \mathrm{kHz}, 2 \mathrm{kHz}$, and $5 \mathrm{kHz}$.

\subsection{Analysis, Interpretation and Discussion of the Results}

The analysis and interpretation of the results presented above are subdivided into three categories: (i) the variation of blocking temperature with change in frequency and with change in particle size; (ii) analysis of the data in the superparamagnetic region of $T>T_{B}$; and (iii) analysis of the data for the region of $T<T_{B}$. The effects of particle size distribution on the measured properties are included in the analysis as appropriate. Since details of the procedures for analyzing the data are similar to those described in Chapter 4 for the analysis of the data for the $7.0 \mathrm{~nm} \gamma-\mathrm{Fe}_{2} \mathrm{O}_{3}$ nanoparticles, these details are omitted here. Instead, the focus is primarily on the results obtained from the analysis.

\subsubsection{Size and Frequency Dependence of the Blocking Temperatures}

In general, the increase in observed $T_{B}$ with increasing particle size (or volume $V$ ) is evident from the definition of $T_{B}$ given in Chapter 2:

$$
T_{B}=\frac{K_{a} V}{k_{B} \ln \left(f_{o} / f_{m}\right)} .
$$

However, from the ZFC and FC $M$ vs. $T$ data for each of the four $\gamma-\mathrm{Fe}_{2} \mathrm{O}_{3}$ samples shown in Fig. 5.1 and listed in Table 5.1, the $T_{B}$ s determined from the peaks in the ZFC data do not show any obvious trend 
with particle diameter $D$. In reality, the observed blocking temperatures depend not only on particle size, but also on applied magnetic field strength and the strength of any interparticle interactions present.

First, the effect of applied magnetic field $H$ is considered. Because the magnetic field used for all the ZFC and FC measurements presented here is only $100 \mathrm{Oe}$, this effect is expected to be negligible. Considering that $H_{k}=2 K_{a} / M_{s}$ with the anisotropy constant $K_{a}$ on the order of $10^{5}-10^{6} \mathrm{erg} / \mathrm{cm}^{3}$, and the saturation magnetization $M_{s} \sim 50^{+} \mathrm{emu} / \mathrm{g}$ for $\gamma-\mathrm{Fe}_{2} \mathrm{O}_{3}$, then $H / H_{k}$ is on the order of $1 / 100$. Because the magnetic field's effect on the relaxation rate is given by (Vallejo-Fernandez, et al., 2013)

$$
f=f_{o} \exp \left\{-\frac{K_{a} V\left[1-H / H_{k}\right]^{2}}{k_{B} T}\right\}
$$

when $H / H_{k}=0.01$, the effect of the field is very small and Eq. 5.2 becomes

$$
f=f_{o} \exp \left\{-\frac{0.98 K_{a} V}{k_{B} T}\right\}
$$

so that $T_{B}$ is $98 \%$ of that given by Eq. 5.1. For the samples investigated here, this effect is within the error of locating the peak in the ZFC data. Furthermore, for the ac magnetic data used to determine the frequency dependence of $T_{B}$, no dc field was used and so there was no reduction of the height of the energy barrier. Therefore, the magnetic field does not explain the lack of a clear trend in the particle size dependence of $T_{B}$ evident from Fig 5.1 or listed in Table 5.1.

\subsubsection{Effects of Interparticle Interactions on the Blocking Temperatures}

From theoretical considerations, computations, and experimental results, it is now accepted that interparticle interactions (IPI) raise the observed blocking temperature $T_{B}$ (Chantrell, et al., 2000; Allia, et al., 2001; Kechrakos \& Trohidu, 1998; Mørup, 1994; Seehra, et al., 2005; Shim, et al., 2008). According to the Vogel-Fulcher relationship, the presence of IPI effectively modifies Eq. 5.1 to (Dorman, et al., 1988; Shtrikman \& Wohlfarth, 1981) the new equation:

$$
T_{B}=T_{o}+\frac{K_{a} V}{k_{B} \ln f_{o} / f_{m}}
$$

where $T_{o}$ is an effective temperature that represents the strength of the interparticle interactions. In order to determine $T_{o}$, the data of the dependence of $T_{B}$ on the measuring frequency $f_{m}$ shown in Fig. 5.5 through Fig. 5.8 is employed again using the peak in $\chi^{\prime \prime}$ for each frequency as the location of $T_{B}$. The values of $T_{B}$ for each $f_{m}$ are plotted in Fig. 5.9 (left) and the fit to Eq. 5.4 is shown as a solid red line. The value of $\left(T_{B}-T_{o}\right)^{-1}$ was plotted vs. $\ln \left(f_{m}\right)$ and fit to a straight line (Fig. 5.9, right) with a slope of $k_{B} / K_{a} V$ and the intercept of $\ln \left(f_{o}\right) k_{B} / K_{a} V$. By keeping $f_{o}=2.6 \times 10^{10} \mathrm{~Hz}$ constant across all four $\gamma$ $\mathrm{Fe}_{2} \mathrm{O}_{3}$ samples, the value of $K_{a}$ could also be determined for each sample. These magnitudes of $T_{o}$ and 

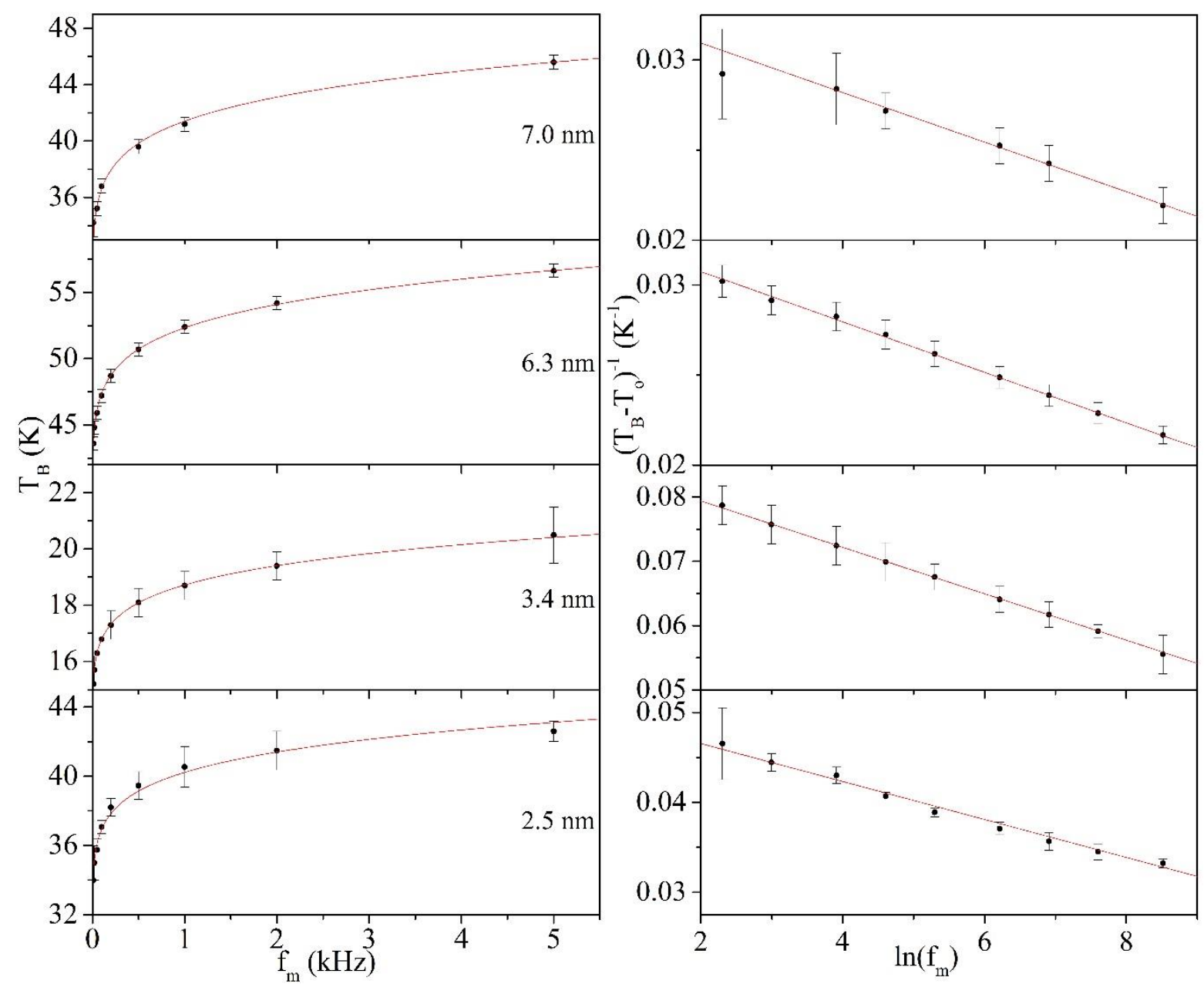

Figure 5.9: Left: $T_{B}$ values determined from the peak in the data of Fig. 5.4. Solid red curve is fit to Eq. 5.4. Right: $\left(T_{B}-T_{o}\right)^{-1} v$ s. $\ln \left(f_{m}\right)$ with red line fit used to determine $T_{a}$ and $\ln \left(f_{o}\right)$.

$K_{a}$ for the four samples are listed in Table 5.2. The size dependence of $K_{a}$ will be discussed later on. For now, the size dependence of $T_{B}$ and $\left(T_{B}-T_{o}\right)$ plotted in Fig. 5.10 shows that $\left(T_{B}-T_{o}\right)$ does vary systematically with increase in the particle size $D$ although the variation of $T_{B}$ with $D$ appears to be quite random. Thus, accounting for the IPI in these particles is quite important for theoretical understanding of the size dependence of the magnetic properties.

Eq. 5.1 gives the blocking temperature as a function of measurement frequency $f_{m}$. An alternative method of determining the presence of interparticle interactions is measuring the change in $T_{B}$ with $\log _{10} f_{m}$ using the equation (Dorman, et al., 1988)

$$
\Phi=\frac{\Delta T_{B}}{T_{B} \Delta \log _{10} f_{m}}
$$




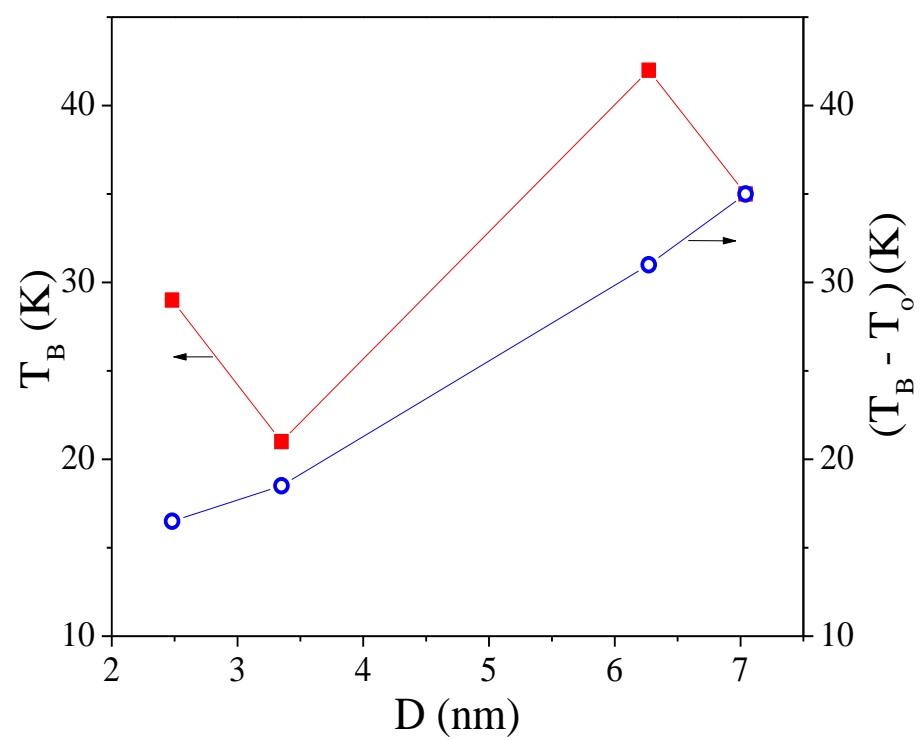

Figure 5.10: $T_{B}$ (red squares) and $T_{B}-T_{o}$ (blue circles) vs. $D$ for $\gamma-F_{2} \mathrm{O}_{3}$ nanoparticle samples. The value of $T_{B}-T_{o}$ increases directly with increasing $D$ while the observed $T_{B}$ values show no clear trend with $D$.

The quantity $\Phi$ is known to vary with IPI strength with $\Phi>0.13$ for non-interacting particles, $\Phi<0.05$ for spin glasses, and $0.13>\Phi>0.05$ for interacting particles with the strength of their interactions increasing with decreasing $\Phi$ (Dorman, et al., 1988). Using the data of the frequency dependence of $T_{B}$ shown in Fig. 5.9 for the four $\gamma-\mathrm{Fe}_{2} \mathrm{O}_{3}$ nanoparticle samples, the magnitudes of $\Phi$ are calculated to be $0.080,0.094,0.084$ and 0.12 for the $2.5 \mathrm{~nm}, 3.4 \mathrm{~nm}, 6.3 \mathrm{~nm}$, and $7.0 \mathrm{~nm}$ samples, respectively compared with the magnitudes of $T_{o}=12.5 \mathrm{~K}, 2.5 \mathrm{~K}, 11.0 \mathrm{~K}$ and $0 \mathrm{~K}$, respectively. These results show that the IPI is the strongest in the $2.5 \mathrm{~nm}$ and $6.3 \mathrm{~nm}$ nanoparticles and essentially absent in the $7.0 \mathrm{~nm}$ nanoparticles.

\subsubsection{Size Dependence of the Anisotropy Constant}

The results listed in Table 5.2 on the size dependence of the anisotropy constant $K_{a}$ show rapid increase in $K_{a}$ with decrease in particle size $D$. Uncertainties in the magnetic anisotropy were determined using

$$
\frac{T_{a}+\text { uncertainty in } T_{a}}{\frac{\pi}{6}\left(\langle D\rangle-\sigma_{D}\right)^{3}}-\frac{T_{a}}{\frac{\pi}{6}\langle D\rangle^{3}}
$$

so that they should represent the maximum possible uncertainty in the anisotropy. This increase in anisotropy with decrease in $D$ is often related to the increasing role of surface anisotropy for smaller particles (Lu, et al., 2007; Fiorani, 2005; Bartolomé, et al., 2008; Pérez, et al., 2008) following the relation (Bødker, et al., 1994):

$$
K_{a}=K_{\text {bulk }}+6 K_{\text {surface }} / D
$$


Table 5.2: Value of $T_{o}$ used for the fitting in Fig. 5.8 and the values of $K_{a}$ calculated from the slope of the linear fit to the plot of $\left(T_{B}-T_{o}\right)^{-1} v s \ln \left(f_{m}\right)$ in Fig 5.8.

\begin{tabular}{|c|c|c|}
\hline$\langle D\rangle(\mathrm{nm})$ & $T_{o}(\mathrm{~K})$ & $K_{a}\left(10^{5} \mathrm{erg} / \mathrm{cm}^{3}\right)$ \\
\hline 7.0 & 0 & $5.57 \pm 1.80$ \\
\hline 6.3 & 11 & $7.51 \pm 1.01$ \\
\hline 3.4 & 2.5 & $18.6 \pm 10$ \\
\hline 2.5 & 12.5 & $80.0 \pm 28$ \\
\hline
\end{tabular}

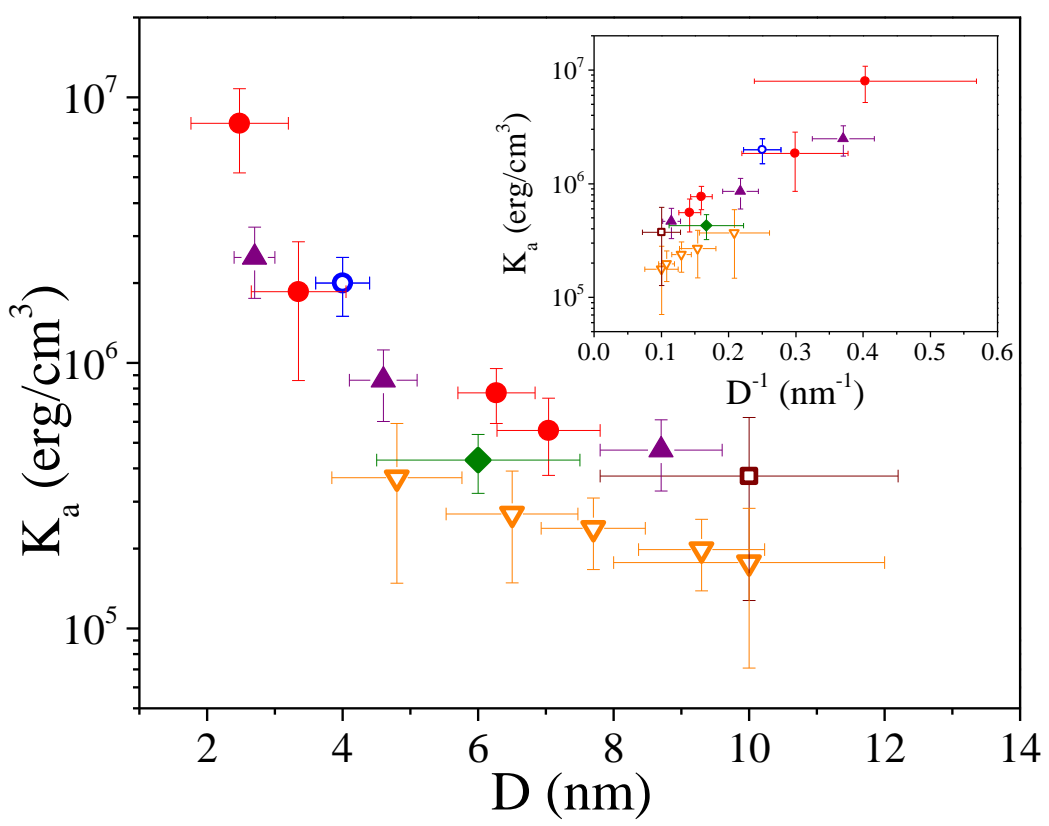

Figure 5.11: Effective anisotropy vs particle diameter. Red circles are data from the $\gamma-\mathrm{Fe}_{2} \mathrm{O}_{3}$ samples presented in this dissertation. Other data shown are from published literature on $\gamma-\mathrm{Fe}_{2} \mathrm{O}_{3}$ nanoparticles: solid purple triangles (Fiorani, et al., 2002), open blue circle (Nadeem, et al., 2011), solid green diamond (Laha, et al., 2014), open orange triangles (Gazeau, et al., 1998), open brown square (Demchenko, et al., 2015). Inset shows the same data plotted against $D^{-1}$.

In Fig. 5.11, the plot of $K_{a}$ vs. $D$ for the maghemite nanoparticles is shown on a semi-log scale with the inset showing variation of $\log K_{a}$ with $1 / D$. In this plot, the literature data reported by others on the variation of $K_{a}$ vs. $D$ for the maghemite nanoparticles is also included. Although the $1 / D$ dependence of $K_{a}$ predicted by Eq. 5.7 is not borne out by the data, there is clearly a more rapid increase in the effective anisotropy with decrease in particle size.

\subsubsection{Analysis of Magnetic Field Dependence of Magnetization above $\boldsymbol{T}_{B}$}

Just as in the case of the $7.0 \mathrm{~nm} \gamma-\mathrm{Fe}_{2} \mathrm{O}_{3}$ nanoparticles in Chapter 4, the data of $M$ vs. $H$ above $T_{B}$ has been analyzed in two ways. First, the data have been fit to a modified Langevin equation that assumes all particles in the sample have the same magnetic moment, $\mu_{p}$ : 


$$
M=M_{o} \mathcal{L}\left[\frac{\mu_{p} H}{k_{B} T}\right]+\chi_{a} H
$$

The results of the fits to Eq. 5.8 for the $7.0 \mathrm{~nm}, 6.3 \mathrm{~nm}, 3.4 \mathrm{~nm}$, and $2.5 \mathrm{~nm} \gamma-\mathrm{Fe}_{2} \mathrm{O}_{3}$ samples are given in Tables 5.3, 5.4, 5.5, and 5.6, respectively. In each case, the data fit reasonably well with Eq. 5.8 at all temperatures shown with the $\mathrm{R}^{2}$ values the lowest for the $150 / 200 \mathrm{~K}$ data of each sample and the poorest fits occurring for the $2.5 \mathrm{~nm}$ sample. The slight increase in $M_{o}$ and $\mu_{p}$ with decreasing temperature can be seen in Fig. 5.12.

The second method involves fitting the $M$ vs. $H$ data to a modified Langevin equation that incorporates a lognormal distribution of magnetic moments:

$$
M=N \frac{1}{s \sqrt{2 \pi}} \int_{0}^{\infty} \mathcal{L}\left(\frac{\mu H}{k_{B} T}\right) \exp \left\{\frac{-\left[\ln \left(\mu / \mu_{o}\right)\right]^{2}}{2 s^{2}}\right\} d \mu+\chi_{a} H
$$

Table 5.3: Parameters for the fit to Eq. 5.12 for the $7.0 \mathrm{~nm} \gamma-\mathrm{Fe}_{2} \mathrm{O}_{3}$ sample

\begin{tabular}{|c|c|c|c|c|}
\hline Temp $(\mathrm{K})$ & $\mathrm{M}_{\mathrm{o}}(\mathrm{emu} / \mathrm{g})$ & $\chi_{\mathrm{a}\left(10^{-5} \mathrm{emu} / \mathrm{gOe}\right)}$ & $\mu_{\mathrm{p}}\left(\mu_{\mathrm{B}}\right)$ & $\mathrm{R}^{2}$ \\
\hline 150 & $51.6 \pm 0.4$ & $3.61 \pm 1.07$ & $7388 \pm 151$ & 0.9983 \\
\hline 200 & $49.8 \pm 0.4$ & $3.56 \pm 1.11$ & $7290 \pm 152$ & 0.9984 \\
\hline 250 & $50.7 \pm 0.4$ & $3.46 \pm 0.98$ & $7325 \pm 131$ & 0.9990 \\
\hline 300 & $47.2 \pm 0.3$ & $3.40 \pm 0.86$ & $6823 \pm 117$ & 0.9993 \\
\hline
\end{tabular}

Table 5.4: Parameters for the fit to Eq. 5.12 for the $6.3 \mathrm{~nm} \gamma-\mathrm{Fe}_{2} \mathrm{O}_{3}$ sample

\begin{tabular}{|c|c|c|c|c|}
\hline Temp $(\mathrm{K})$ & $\mathrm{M}_{\mathrm{o}}(\mathrm{emu})$ & $\chi_{\mathrm{a}\left(10^{-5} \mathrm{emu} / \mathrm{Oe}\right)}$ & $\mu_{\mathrm{p}}\left(\mu_{\mathrm{B}}\right)$ & $\mathrm{R}^{2}$ \\
\hline 200 & $47.9(0.3)$ & $7.3(0.6)$ & $8456(175)$ & 0.9964 \\
\hline 250 & $46.1(0.3)$ & $6.9(0.5)$ & $8405(165)$ & 0.9972 \\
\hline 300 & $43.9(0.3)$ & $6.6(0.5)$ & $8111(161)$ & 0.9978 \\
\hline 350 & $42.1(0.3)$ & $6.6(0.6)$ & $7716(158)$ & 0.9980 \\
\hline
\end{tabular}

Table 5.5: Parameters for the fit to Eq. 5.12 for the $3.4 \mathrm{~nm} \gamma-\mathrm{Fe}_{2} \mathrm{O}_{3}$ sample

\begin{tabular}{|c|c|c|c|c|}
\hline Temp $(\mathrm{K})$ & $\mathrm{M}_{\mathrm{o}}(\mathrm{emu} / \mathrm{g})$ & $\chi_{\mathrm{a}\left(10^{-5} \mathrm{emu} / \mathrm{gOe}\right)}$ & $\mu_{\mathrm{p}}\left(\mu_{\mathrm{B}}\right)$ & $\mathrm{R}^{2}$ \\
\hline 150 & $53.5(0.6)$ & $4.80(0.95)$ & $1779(46)$ & 0.99830 \\
\hline 200 & $51.6(0.3)$ & $4.65(0.55)$ & $1728(24)$ & 0.99950 \\
\hline 250 & $49.9(0.2)$ & $4.72(0.38)$ & $1689(17)$ & 0.99978 \\
\hline 300 & $46.2(0.2)$ & $4.78(0.33)$ & $1623(15)$ & 0.99984 \\
\hline
\end{tabular}

Table 5.6: Parameters for the fit to Eq. 5.12 for the $2.5 \mathrm{~nm} \gamma-\mathrm{Fe}_{2} \mathrm{O}_{3}$ sample

\begin{tabular}{|c|c|c|c|c|}
\hline Temp $(\mathrm{K})$ & $\mathrm{M}_{\mathrm{o}}(\mathrm{emu} / \mathrm{g})$ & $\chi_{\mathrm{a}\left(10^{-5} \mathrm{emu} / \mathrm{g} \text { Oe }\right)}$ & $\mu_{\mathrm{p}}\left(\mu_{\mathrm{B}}\right)$ & $\mathrm{R}^{2}$ \\
\hline 200 & $32.6(1.1)$ & $9.80(1.79)$ & $2692(214)$ & 0.97647 \\
\hline 250 & $30.7(0.9)$ & $9.88(1.42)$ & $2628(173)$ & 0.98314 \\
\hline 300 & $28.6(0.6)$ & $9.88(0.99)$ & $2555(125)$ & 0.98891 \\
\hline 350 & $25.9(1.0)$ & $9.73(1.52)$ & $2500(203)$ & 0.98368 \\
\hline
\end{tabular}




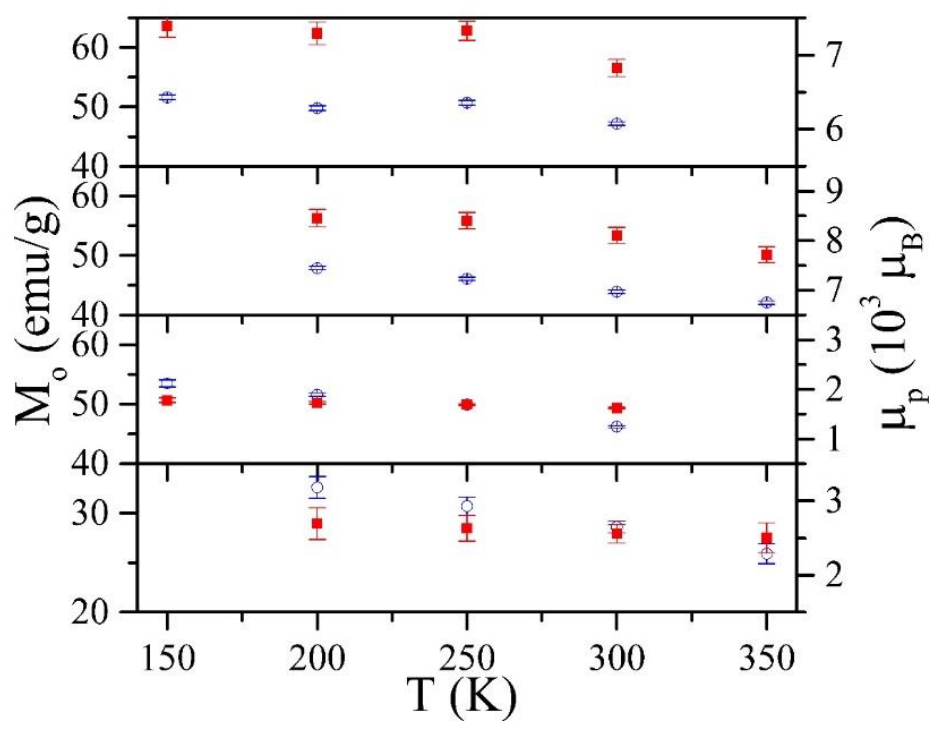

Figure 5.12: Fitting parameters $M_{o}$ (blue circles) and $\mu_{p}$ (red squares) of Tables 5.3, 5.4, 5.5, and 5.6. Top to bottom: $7.0 \mathrm{~nm}, 6.3 \mathrm{~nm}, 3.4 \mathrm{~nm}$, and $2.5 \mathrm{~nm}$ samples.

Due to the number of parameters in Eq. 5.9, some constraints are necessary in order to initialize the fitting function. First, the number of particles per unit mass is considered. The average mass per particle is calculated using

$$
\frac{\pi}{6}\left\{\rho_{m}\langle D\rangle^{3}+\rho_{O A}\left[(\langle D\rangle+2 t)^{3}-\langle D\rangle^{3}\right]\right\}
$$

where $\rho_{m}=4.856 \mathrm{~g} / \mathrm{cm}^{3}$ is the density of $\gamma-\mathrm{Fe}_{2} \mathrm{O}_{3}, \rho_{O A}=0.895 \mathrm{~g} / \mathrm{cm}^{3}$ is the density of oleic acid, $\langle D\rangle$ is the mean diameter of $\gamma-\mathrm{Fe}_{2} \mathrm{O}_{3}$ in the sample as determined by TEM, and $t$ is the thickness of the oleic acid coating estimated from the TGA data in Chapter 3. The number of particles per unit mass can be found by taking the reciprocal of Eq. 5.10 and multiplying by the packing efficiency, $\alpha$ :

$$
N=\alpha \frac{6}{\pi}\left\{\rho_{O A}\left[(\langle D\rangle+2 t)^{3}-\langle D\rangle^{3}\right]+\rho_{\text {maghemite }}\langle D\rangle^{3}\right\}^{-1} .
$$

For identical spheres, the highest possible packing efficiency occurs for a hexagonal close paced (HCP) stacking where $\alpha=\pi / 3 \sqrt{2}$, or approximately $74 \%$ of the space is occupied by the nanoparticles. The calculated values for perfectly efficient packing, $N_{1}$, and HCP stacking are given in Table 5.7. For each

Table 5.7: The quantity $N_{1}$ is the maximum possible number of particles per unit mass assuming 100\% packing efficiency (impossible). $N_{H C P}$ is calculated assuming a HCP arrangement of identical spheres.

\begin{tabular}{|c|c|c|}
\hline$\langle D\rangle(\mathrm{nm})$ & $\mathrm{N}_{1}\left(10^{17} \mathrm{~g}^{-1}\right)$ & $\mathrm{N}_{\mathrm{HCP}}\left(10^{17} \mathrm{~g}^{-1}\right)$ \\
\hline 7.0 & 9.5 & 7.0 \\
\hline 6.3 & 11.8 & 8.7 \\
\hline 3.4 & 71.4 & 52.8 \\
\hline 2.5 & 190.5 & 141.0 \\
\hline
\end{tabular}


fitting attempt an initial value of $N_{H C P}$ was used to initialize the fitting with the exception of the $7.0 \mathrm{~nm}$ data for which $N \approx\left(\rho_{m} \pi\langle D\rangle^{3} / 6\right)^{-1}$ was used to initialize. In each case, the value of $N$ was not allowed to reach $N_{1}$. Because the samples analyzed in this dissertation have log normal size distributions and the oleic acid coatings included in the total particle diameter for the calculation of $N$ are not rigid, $N>N_{H C P}$ is allowed so long as $\alpha<1$.

Next, $\mu_{o}$ was initialized at the value of $\mu_{p}$ found from the single moment modified Langevin fit of the previous section. For initialization, $\chi_{a}$ was held constant at the value determined in the previous section and the fitting procedure was allowed to run until the fit converged or 100 iterations had been completed. Then, with an initial fit complete within a range of reasonable values, all parameters were allowed to vary. A Levenberg-Marquardt algorithm was used to determine the least squares fit for the curve and the results are shown as solid curves in Fig. 5.4. The parameters from the fits shown in Fig. 5.4 are given in Tables 5.8, 5.9, 5.10, and 5.11 for the $7.0 \mathrm{~nm}, 6.3 \mathrm{~nm}, 3.4 \mathrm{~nm}$, and $2.5 \mathrm{~nm}$ samples, respectively.

Table 5.8: Parameters from the fit of Eq. 5.9 to the $M$ vs. $\mathrm{H}$ data for $7.0 \mathrm{~nm} \gamma-\mathrm{Fe}_{2} \mathrm{O}_{3}$ nanoparticles

\begin{tabular}{|c|c|c|c|c|c|c|c|}
\hline $\begin{array}{c}\text { Temp } \\
(\mathrm{K})\end{array}$ & $\begin{array}{c}N \\
\left(10^{17} \mathrm{~g}^{-1}\right)\end{array}$ & $\begin{array}{c}\mu_{o} \\
\left(\mu_{\mathrm{B}}\right)\end{array}$ & $s$ & $\begin{array}{c}\langle\mu\rangle \\
\left(\mu_{\mathrm{B}}\right)\end{array}$ & $\begin{array}{c}M_{S}=N\langle\mu\rangle \\
(\mathrm{emu} / \mathrm{g})\end{array}$ & $\begin{array}{c}\chi_{a}\left(10^{-5}\right. \\
\mathrm{emu} / \mathrm{g} \mathrm{Oe})\end{array}$ & $\mathrm{R}^{2}$ \\
\hline 150 & $8.37 \pm 0.10$ & $5958 \pm 5$ & $0.475 \pm 0.016$ & $6670 \pm 55$ & $51.8 \pm 1.1$ & $3.59 \pm 0.64$ & 0.9990 \\
\hline 200 & $8.37 \pm 0.20$ & $5750 \pm 216$ & $0.478 \pm 0.036$ & $6554 \pm 364$ & $50.9 \pm 4.1$ & $3.59 \pm 0.39$ & 0.9995 \\
\hline 250 & $8.36 \pm 0.08$ & $5846 \pm 5$ & $0.480 \pm 0.013$ & $6560 \pm 46$ & $50.9 \pm 0.8$ & $5.40 \pm 0.53$ & 0.9992 \\
\hline 300 & $8.35 \pm 0.07$ & $5463 \pm 5$ & $0.470 \pm 0.011$ & $6101 \pm 36$ & $47.2 \pm 0.7$ & $3.37 \pm 0.36$ & 0.9995 \\
\hline
\end{tabular}

Table 5.9: Parameters from the fit of Eq. 5.9 to the $M v s . H$ data for $6.3 \mathrm{~nm} \gamma-\mathrm{Fe}_{2} \mathrm{O}_{3}$ nanoparticles

\begin{tabular}{|c|c|c|c|c|c|c|c|}
\hline $\begin{array}{c}\text { Temp } \\
(\mathrm{K})\end{array}$ & $\begin{array}{c}N \\
\left(10^{17} \mathrm{~g}^{-1}\right)\end{array}$ & $\begin{array}{c}\mu_{o} \\
\left(\mu_{\mathrm{B}}\right)\end{array}$ & $s$ & $\begin{array}{c}\langle\mu\rangle \\
\left(\mu_{\mathrm{B}}\right)\end{array}$ & $\begin{array}{c}M_{S}=N\langle\mu\rangle \\
(\mathrm{emu} / \mathrm{g})\end{array}$ & $\begin{array}{c}\chi_{a}\left(10^{-5}\right. \\
\mathrm{emu} / \mathrm{g} \mathrm{Oe})\end{array}$ & $\mathrm{R}^{2}$ \\
\hline 200 & $7.93 \pm 0.09$ & $5281 \pm 91$ & $0.670 \pm 0.012$ & $6610 \pm 4975$ & $48.6 \pm 37.6$ & $8.54 \pm 0.18$ & 0.99985 \\
\hline 250 & $8.05 \pm 0.10$ & $5047 \pm 100$ & $0.674 \pm 0.013$ & $6334 \pm 4803$ & $47.3 \pm 36.9$ & $6.50 \pm 0.14$ & 0.99986 \\
\hline 300 & $8.11 \pm 0.13$ & $4866 \pm 117$ & $0.657 \pm 0.015$ & $6038 \pm 4436$ & $45.4 \pm 34.6$ & $5.35 \pm 0.15$ & 0.99986 \\
\hline 350 & $8.11 \pm 0.14$ & $4786 \pm 117$ & $0.624 \pm 0.016$ & $5815 \pm 4012$ & $43.7 \pm 31.5$ & $5.15 \pm 0.17$ & 0.99989 \\
\hline
\end{tabular}

Table 5.10: Parameters from the fit of Eq. 5.9 to the $M v s . H$ data for $3.4 \mathrm{~nm} \gamma-\mathrm{Fe}_{2} \mathrm{O}_{3}$ nanoparticles

\begin{tabular}{|c|c|c|c|c|c|c|c|}
\hline $\begin{array}{c}\text { Temp } \\
(\mathrm{K})\end{array}$ & $\begin{array}{c}N \\
\left(10^{18} \mathrm{~g}^{-1}\right)\end{array}$ & $\begin{array}{c}\mu_{o} \\
\left(\mu_{\mathrm{B}}\right)\end{array}$ & $s$ & $\langle\mu\rangle\left(\mu_{\mathrm{B}}\right)$ & $\begin{array}{c}M_{S}=N\langle\mu\rangle \\
(\mathrm{emu} / \mathrm{g})\end{array}$ & $\begin{array}{c}\chi_{a}\left(10^{-5}\right. \\
\mathrm{emu} / \mathrm{g} \mathrm{Oe})\end{array}$ & $\mathrm{R}^{2}$ \\
\hline 150 & $3.81 \pm 0.11$ & $1375 \pm 66$ & $0.490 \pm 0.039$ & $1550 \pm 807$ & $54.8 \pm 30.9$ & $3.60 \pm 0.53$ & 0.99967 \\
\hline 200 & $3.81 \pm 0.07$ & $1347 \pm 23$ & $0.454 \pm 0.015$ & $1494 \pm 715$ & $52.8 \pm 26.7$ & $3.35 \pm 0.18$ & 0.99997 \\
\hline 250 & $3.80 \pm 0.04$ & $1325 \pm 11$ & $0.432 \pm 0.007$ & $1455 \pm 659$ & $51.3 \pm 24.0$ & $3.26 \pm 0.08$ & 0.99999 \\
\hline 300 & $3.80 \pm 0.04$ & $1231 \pm 8$ & $0.445 \pm 0.005$ & $1359 \pm 636$ & $47.9 \pm 23.1$ & $2.95 \pm 0.06$ & 0.99999 \\
\hline
\end{tabular}


Table 5.11: Parameters from the fit of Eq. 5.9 to the $M$ vs. $\mathrm{H}$ data for $2.5 \mathrm{~nm} \gamma-\mathrm{Fe}_{2} \mathrm{O}_{3}$ nanoparticles

\begin{tabular}{|c|c|c|c|c|c|c|c|}
\hline $\begin{array}{c}\text { Temp } \\
(\mathrm{K})\end{array}$ & $\begin{array}{c}N \\
\left(10^{18} \mathrm{~g}^{-1}\right)\end{array}$ & $\begin{array}{c}\mu_{o} \\
\left(\mu_{\mathrm{B}}\right)\end{array}$ & $s$ & $\begin{array}{c}\langle\mu\rangle \\
\left(\mu_{\mathrm{B}}\right)\end{array}$ & $\begin{array}{c}M_{S}=N\langle\mu\rangle \\
(\mathrm{emu} / \mathrm{g})\end{array}$ & $\begin{array}{c}\chi_{a}\left(10^{-5}\right. \\
\mathrm{emu} / \mathrm{g} \mathrm{Oe})\end{array}$ & $\mathrm{R}^{2}$ \\
\hline 200 & $2.39 \pm 0.46$ & $1132 \pm 312$ & $0.847 \pm 0.132$ & $1620 \pm 1659$ & $35.9 \pm 50.8$ & $6.30 \pm 1.38$ & 0.9925 \\
\hline 250 & $2.36 \pm 0.39$ & $1108 \pm 277$ & $1.000 \pm 0.104$ & $1826 \pm 2394$ & $40.0 \pm 67.6$ & $5.00 \pm 0.84$ & 0.9970 \\
\hline 300 & $2.40 \pm 0.50$ & $938 \pm 276$ & $0.900 \pm 0.131$ & $1406 \pm 1571$ & $31.3 \pm 48.8$ & $6.70 \pm 1.01$ & 0.9966 \\
\hline 350 & $2.12 \pm 0.60$ & $985 \pm 362$ & $0.883 \pm 0.155$ & $1455 \pm 1581$ & $28.6 \pm 48.0$ & $6.63 \pm 1.63$ & 0.9940 \\
\hline
\end{tabular}

\subsubsection{Discussion of Modified Langevin Fit Results}

The higher $\mathrm{R}^{2}$ values obtained for the modified Langevin fit that included a distribution of magnetic moments is to be expected from the increased parameterization in Eq. 5.9 over Eq. 5.8. More interesting is the difference between magnetic moments for each particle determined in these cases. For the $7.0 \mathrm{~nm}$ and $3.4 \mathrm{~nm}$ samples, the mean magnetic moment $\langle\mu\rangle$ is comparable to the value of $\mu_{p}$ determined at each temperature. The values for the $6.3 \mathrm{~nm}$ and $2.5 \mathrm{~nm}$ samples, however, do not agree as well. When the value of $s$ is large as is the case for the $6.3 \mathrm{~nm}$ and $2.5 \mathrm{~nm}$ samples, the single moment modified Langevin fit gives an overestimate of the magnetic moment. For the $7.0 \mathrm{~nm}$ and $2.5 \mathrm{~nm}$ samples, the value of $s$ is close to three times the diameter distribution width $\lambda_{D}$ indicating that the distribution of particle magnetic moments can be largely attributed to the distribution in particle volumes. In the case of the $3.4 \mathrm{~nm}$ and $6.3 \mathrm{~nm}$ samples, however, the values of $s$ deviate significantly from the volume distribution widths. For the $3.4 \mathrm{~nm}$ sample, $s<3 \lambda_{D}$ and the results of the two fittings agree quite well. On the other hand, the $6.3 \mathrm{~nm}$ sample has $s>3 \lambda_{D}$ indicating that size alone does not explain the distribution of particle magnetic moments. It is interesting to note that the $6.3 \mathrm{~nm}$ sample was the only one that exhibited noticeable hysteresis. A possible explanation for this is that this particular sample has some larger particles present that were not detected in the TEM analysis.

\subsubsection{Temperature Dependence of dc Magnetic Susceptibility above $\boldsymbol{T}_{B}$}

In Chapter 4, the superparamagnetic susceptibility in the low-field, high temperature limit was shown to the follow Eq. 4.8 which, for ease of discussion, is rewritten below:

$$
\chi=M / H=\chi_{o}+\frac{C}{T}
$$

After determining $\chi_{o}$ in the manner described in Chapter 4, the plots of $\left(\chi-\chi_{o}\right)^{-1}$ vs. $T$ for the four samples are obtained and shown in Fig. 5.13 and the magnitudes of the evaluated parameters such as $\mu_{p}$ are given in Table 5.12. As noted in Chapter 4, these magnitudes of $\mu_{p}$ tend to be larger than those determined using modified Langevin function of Eq. 5.8. This is because in the low-field, high temperature limit, the dominant contribution to $\mu_{p}$ comes from the larger particles in the distribution. Despite this limitation, the general validity of Eq. 5.12 for all four $\gamma-\mathrm{Fe}_{2} \mathrm{O}_{3}$ is established. According to 


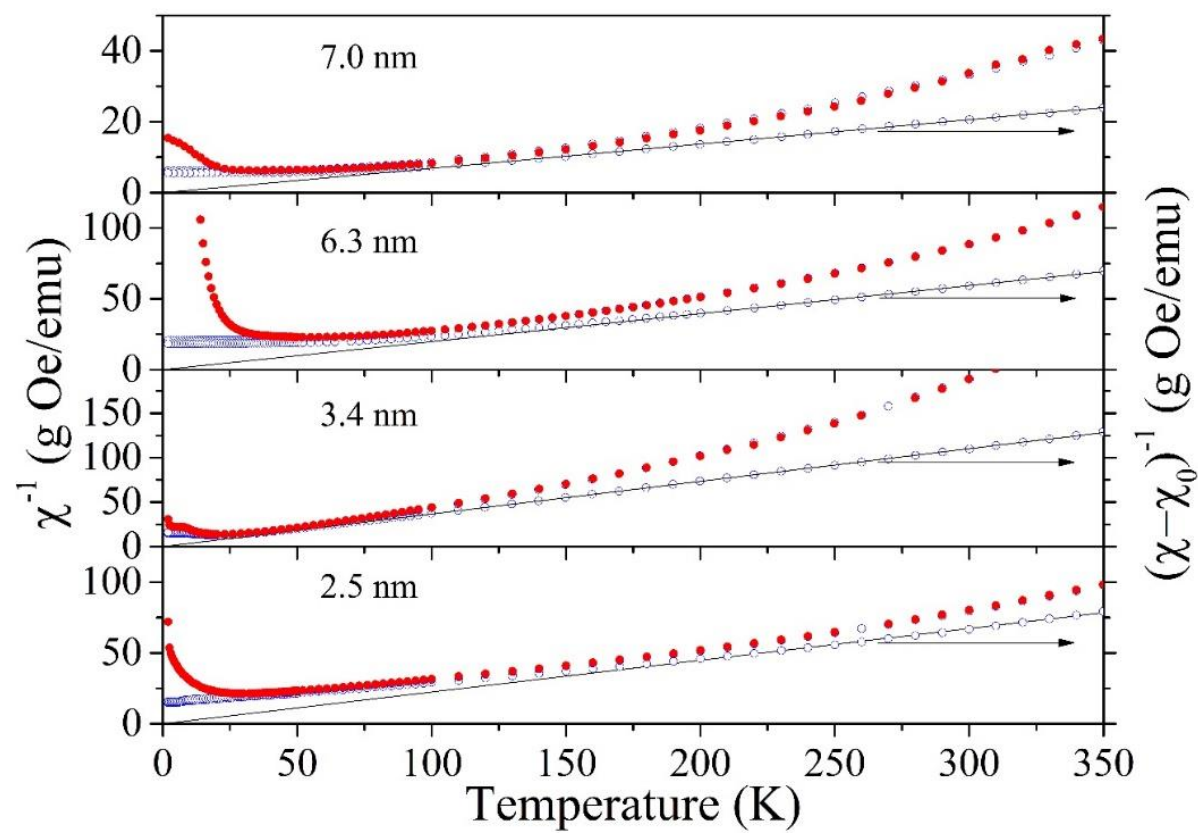

Figure 5.13: Curie-Weiss fit to the data of $M v s T$ in a 100 Oe measuring field. Solid red circles are $Z F C$ data and open blue circles are FC data. Solid line is a linear fit to the high temperature data of $\chi-\chi_{o}$ illustrating the adherence to the $C W$ law for high temperatures. The values of $\chi_{o}$ and $C$ determined from this fit are given in Table 5.2.

Table 5.12: Results of the Curie-Weiss fit to the data of Fig. 5.13.

\begin{tabular}{|c|c|c|c|}
\hline$\langle D\rangle(\mathrm{nm})$ & $C(\mathrm{emu} \mathrm{K} / \mathrm{g} \mathrm{Oe})$ & $\chi_{o}\left(10^{-3} \mathrm{emu} / \mathrm{g} \mathrm{Oe}\right)$ & $\mu_{p}\left(\mu_{B}\right)$ \\
\hline 7.0 & $14.59(0.01)$ & $-18.6(0.1)$ & $10027 \pm 140$ \\
\hline 6.3 & $5.05(0.01)$ & $-5.6(0.1)$ & $4026 \pm 30$ \\
\hline 3.4 & $2.37(0.01)$ & $-3.8(0.1)$ & $1706 \pm 82$ \\
\hline 2.5 & $4.46(0.01)$ & $-2.4(0.2)$ & $4977 \pm 126$ \\
\hline
\end{tabular}

Eq. 5.12, the superparamagnetic susceptibility does not follow the Curie law but a modified Curie law with information on $\mu_{p}$ contained in $C$.

\subsection{Relationship between $T_{o}$ and $\Phi$}

From the discussion presented in this chapter, it is evident that both $T_{o}$ and $\Phi$ of Eq. 5.4 and Eq. 5.5, respectively, provide a measure of the strength of interparticle interactions in magnetic nanoparticles. Therefore, it is natural to ask if there is a relationship between these two parameters. Here, such a relationship is derived and verified using the data on the $\gamma-\mathrm{Fe}_{2} \mathrm{O}_{3}$ nanoparticles investigated in this dissertation as well as data on other magnetic nanoparticles available in the literature.

To determine the relationship between $T_{o}$ of Eq. 5.4 and $\Phi$ of Eq. 5.5, first, the quantity $\Delta T_{B}$ is considered. For a single sample, let $T_{B 1}$ be the blocking temperature observed at $f_{m 1}$ and $T_{B 2}$ be the blocking temperature observed at $f_{m 2}$, given by 


$$
T_{B 1}=T_{o}+\frac{T_{a}}{\ln \left(f_{o} / f_{m 1}\right)}
$$

and

$$
T_{B 2}=T_{o}+\frac{T_{a}}{\ln \left(f_{o} / f_{m 2}\right)},
$$

respectively. Then, $\Delta T_{B}=T_{B 2}-T_{B 1}$ can be written as

$$
\Delta T_{B}=T_{a} \frac{\ln \left(f_{m 2}\right)-\ln \left(f_{m 1}\right)}{\ln \left(f_{o} / f_{m 1}\right) \ln \left(f_{o} / f_{m 2}\right)}
$$

Dividing Eq. 5.15 by $T_{B 1} \log \left(f_{m 2} / f_{m 1}\right)$ to get $\Phi$ yields

$$
\Phi=\Phi_{o}\left[1-\frac{T_{o}}{T_{B 1}}\right]
$$

where

$$
\Phi_{o}=\frac{2.30}{\ln \left(f_{o} / f_{m 2}\right)}
$$

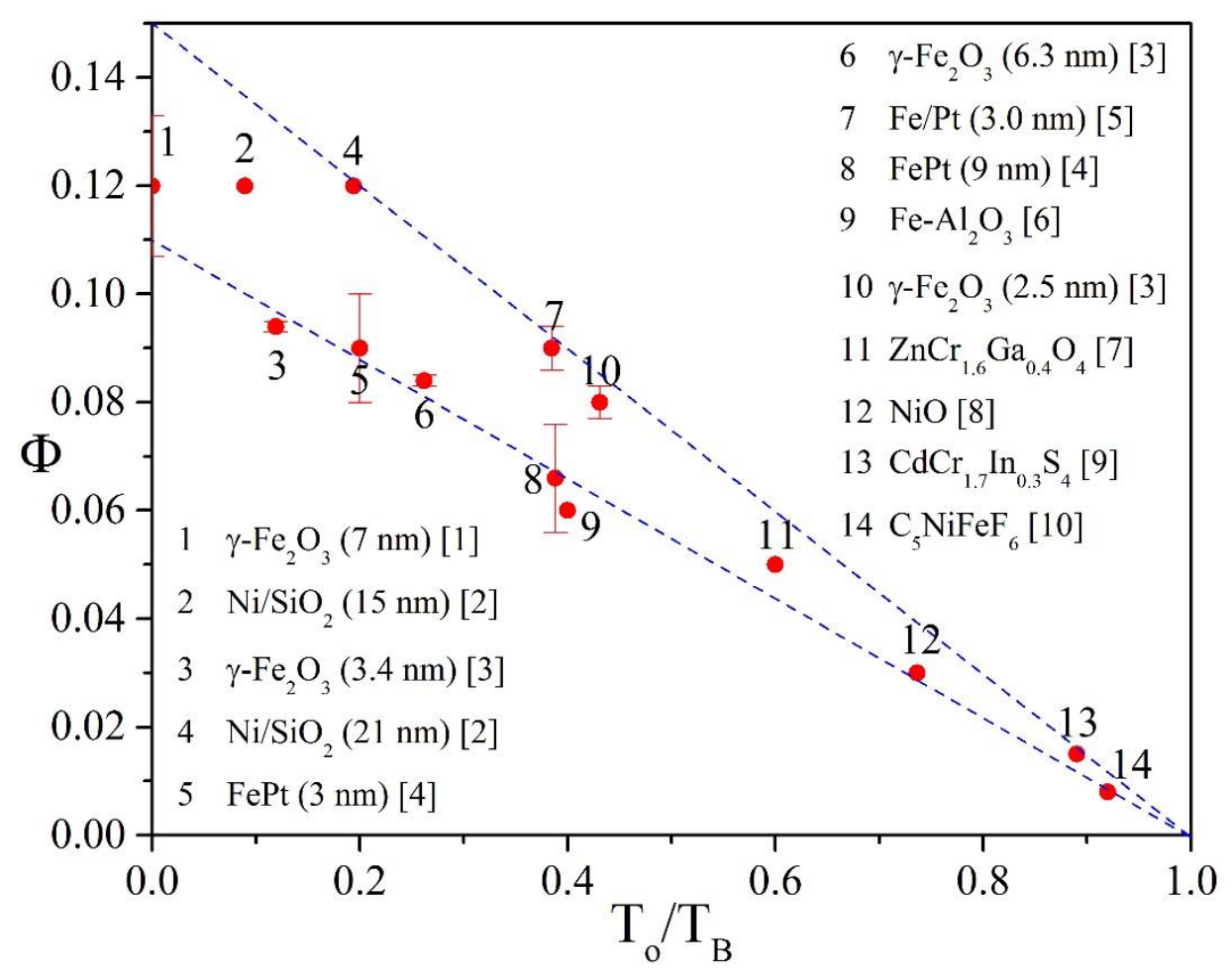

Figure 5.14: Interparticle interaction parameter $\Phi$ vs. $T_{o} / T_{B}$. The dashed lines represent $\Phi_{o}=0.11$ and 0.15. The references for data are: [1] (Pisane, et al., 2015), [2] (Singh, et al., 2009), [3] data from this dissertation to be published, [4] (Seehra, et al., 2010), [5] (Pisane, et al., 2015), [6] (Dorman, et al., 1988), [7] (Fiorani, et al., 1984), [8] (Shim, et al., 2006), [9] (Vincent, et al., 1986), and [10] (Pappa, et al., 1985). 
Table 5.13: Summary of key properties of maghemite nanoparticles

\begin{tabular}{|c|c|c|c|c|c|c|}
\hline$\langle D\rangle(\mathrm{nm})$ & $\sigma_{D}(\mathrm{~nm})$ & $\mathrm{T}_{\mathrm{B}}(\mathrm{K})$ & $\mathrm{T}_{\mathrm{o}}(\mathrm{K})$ & $\mathrm{T}_{\mathrm{B}}-\mathrm{T}_{\mathrm{o}}(\mathrm{K})$ & $\mathrm{K}_{\mathrm{a}}\left(10^{5} \mathrm{emu} / \mathrm{cm}^{3}\right)$ & $\Phi$ \\
\hline 2.5 & 0.7 & 29 & 12.5 & 16.5 & $79.95 \pm 28$ & $0.080 \pm 0.003$ \\
\hline 3.4 & 0.7 & 21 & 2.5 & 18.5 & $18.57 \pm 10$ & $0.094 \pm 0.001$ \\
\hline 6.3 & 0.6 & 42 & 11 & 31 & $7.51 \pm 1.01$ & $0.084 \pm 0.001$ \\
\hline 7.0 & 0.8 & 35 & 0 & 35 & $5.57 \pm 1.80$ & $0.120 \pm 0.013$ \\
\hline
\end{tabular}

If $f_{m 2} \cong 200 \mathrm{~Hz}$, and $f_{o} \cong 10^{10} \mathrm{~Hz}$, then $\Phi_{o}=0.13$ for the non-interacting particle case $\left(T_{o}=0 \mathrm{~K}\right)$ and $\Phi$ will decrease with increasing interparticle interactions (increasing $T_{o}$ ). Typical values of $\Phi$ used are: $\Phi>0.13$ for non-interacting particles, $\Phi<0.05$ for spin glasses, and $0.13>\Phi>0.05$ for interacting particles with the strength of their interactions increasing with decreasing $\Phi$. However, from Eq. 5.17, it is clear that the actual value of $\Phi$ for $T_{o}=0 \mathrm{~K}$ will depend on $f_{o}$ and $f_{m 2}$. The threshold for the onset of IPI is then $\Phi=\Phi_{o}$. In Fig. $5.14 \Phi$ values for the samples presented in this dissertation as well as from the literature are plotted against $\left(T_{o} / T_{B}\right)$. The dashed red lines indicate values of $\Phi_{o}=0.11$ and 0.15 . The data shown follow the relationship quite well with most of the data points falling along between the dashed red lines.

\subsection{Summary}

In this chapter, the magnetic properties of four $\gamma-\mathrm{Fe}_{2} \mathrm{O}_{3}$ nanoparticle samples have been presented and the analysis of the data discussed. Some key properties of the samples are summarized in Table 5.13. The notable results include the determination of the Vogel-Fulcher temperatures and the development of the mathematical relationship between $T_{o}$ and $\Phi$. In addition, the theoretical basis for the value of $\Phi$ that indicates the onset of interparticle interactions given here has not, to date, been explained elsewhere. The trend in magnetic anisotropy with nanoparticle diameter observed for $\gamma-\mathrm{Fe}_{2} \mathrm{O}_{3}$ nanoparticles, when careful consideration is given to the effects of interparticle interactions, illustrates strong dependence of the magnetic anisotropy on the particle size and illustrates that the commonly used $K=K_{b u l k}+$ $6 K_{\text {surface }} / D$ relationship is invalid for spherical maghemite nanoparticles. In addition, data from the literature is used to verify the relationships between $T_{o}$ and $\Phi$ and between $K_{a}$ and $D$. The importance of a narrow size distribution for utilizing the modified Langevin analysis is further illustrated in the comparison of the two different modified Langevin fits to the data. 


\section{Chapter 6}

\section{Iron-Platinum Core-Shell Nanoparticles: Synthesis, Structural Characterization, and Magnetic Properties}

\subsection{Introduction}

In this chapter the synthesis, structural characterization, and magnetic properties of $3.1 \mathrm{~nm}$ ironplatinum (Fe-Pt) core-shell magnetic nanoparticles are discussed. The techniques employed follow the procedures of Chapters 3 and 4 for the structural and magnetic characterization, respectively. The data presented in this chapter are all from a single sample of Fe-Pt core-shell nanoparticles that also included a considerable amount of the stabilizing agent polyvinylpyrollidone left behind from the synthesis. The results presented in this chapter have been published by the author recently (Pisane, et al., 2015) and also presented at the 2014 Institute of Electrical and Electronics Engineers Magnetism and Magnetic Materials Conference.

\subsection{Background}

Precious metals including platinum, palladium, and rhodium are used in catalytic reactions such as those in automobile catalytic converters (Gandhi, et al., 2003); however, they are expensive and are often imported so reducing the amount needed for these applications is important. One method for reducing the amount of precious metals needed for catalytic reactions is the implementation of core-shell nanostructures with inexpensive core materials such as iron. In this way, the precious metals are all located on surfaces which is where the catalytic reactions take place. Core-shell nanoparticles have properties that depend not only on the constituent materials, but also the ratio of the core to shell materials (Chaudhuri \& Paria, 2012; Kalele, et al., 2006) in addition to the effects of finite size that impact nanoparticle behavior. The studies relevant to the results presented here are those on the core-shell nanoparticles of CoO-Pt (Zeleňáková, et al., 2014), Fe-Au (Carpenter, 2001), Fe-Ag (Lu, et al., 2010), Co-Pt (Park \& Cheon, 2001), Fe- $\gamma-\mathrm{Fe}_{2} \mathrm{O}_{3}$ (Singh, et al., 2011), and studies on Fe nanoparticles of various sizes (Kneller \& Luborsky, 1963; Monson, et al., 2013; Ibusuki, et al., 2001; Xiao, et al., 1986; Singh, et al., 2011).

\subsection{Synthesis of Fe-Pt Core-Shell Nanoparticles}

\subsubsection{Chemicals \& Equipment Required for Synthesis of Fe-Pt Core-Shell Nanoparticles}

In the synthesis of Fe-Pt core-shell nanoparticles, the following chemicals were purchased from Alfa Aesar and used as received without further modification:

- $\quad$ iron acetylacetonate (stock \#12534) $-40 \mathrm{mg}$ 
- $\quad$ ethylene glycol (stock \#A11591)-20 mg

- polyvinylpyrrolidone (stock \#J60382)-25 mg

- $\quad$ platinum chloride (stock \#11044)-45 mg

- $\quad$ hexane (stock \#L09938)-25 mL

All steps in the synthesis procedure were performed in a fume hood. The condenser was cooled by slowly running tap water and the annealing took place under flowing high purity nitrogen gas (Matheson TriGas). The equipment required for synthesis included:

- round-bottomed flask

- condenser

- thermometer

- heating pad

- graduated cylinder

- scale

- glass boat

- tube furnace

The equipment setup for synthesis was the same as described in Chapter 3 for the $\gamma-\mathrm{Fe}_{2} \mathrm{O}_{3}$ synthesis.

\subsubsection{Synthesis Procedure}

The Fe-Pt core-shell nanoparticles were synthesized using a sequential polyol process modified from the procedures of Alayoglu, et al. (2008) for synthesizing Ru-Pt core-shell nanoparticles and Zhou, et al. (2005) for synthesizing Cu-Pt core-shell nanoparticles. First, $40 \mathrm{mg}$ iron acetylacetonate, $20 \mathrm{mg}$ of ethylene glycol, and $25 \mathrm{mg}$ of polyvinylpyrrolidone were combined in a round bottom flask connected to a condenser and the mixture was heated to reflux $\left(160-180{ }^{\circ} \mathrm{C}\right)$ and held there for 1 hour as the iron cores formed. Then, the solution was allowed to cool to room temperature before $45 \mathrm{mg}$ of platinum chloride was added. The mixture was heated to reflux again and this time the temperature was maintained for 3 hours for the formation of the platinum shell. Next, the solution was allowed to cool to room temperature before hexane was added to aid in the precipitation of the nanoparticles. The resulting slurry was poured into glass boats and placed in a tube furnace under flowing nitrogen gas. The temperature was raised to $200{ }^{\circ} \mathrm{C}$ and maintained for 2 hours. The particles were collected from the boat using a small amount of ethanol and a spatula to gently scrape the powder from the bottom of the boat. The ethanol was then allowed to evaporate off by leaving the nanoparticle/ethanol suspension in a watch glass. The synthesis procedure is summarized in Fig. 6.1. 


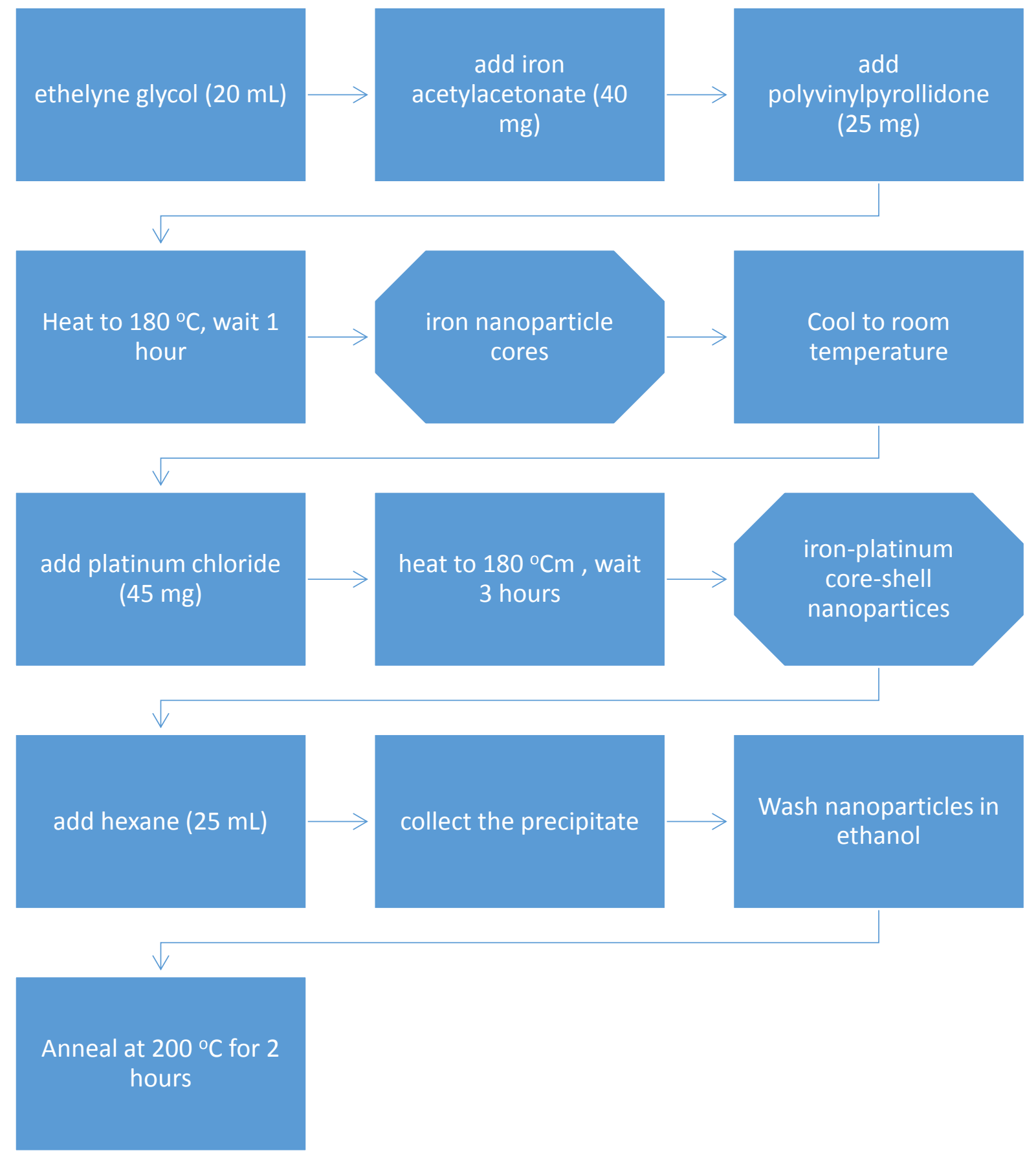

Figure 6.1: Synthesis procedure for the Fe-Pt core-shell nanoparticles. The octagonal sections indicate the formation of iron nanoparticles and the finished core-shell particles. 


\subsection{Structural Characterization}

\subsubsection{Transmission Electron Microscopy and X-ray Diffraction}

The particle areas were determined from TEM micrographs following the procedure outlined in Chapter 3. A representative micrograph and a histogram of the calculated diameters is shown in Fig. 6.2 with the fit to a lognormal distribution is shown as a solid red curve. From this fit, the parameters $D_{o}=3.0 \mathrm{~nm}$ and $\lambda_{D}=0.31$ were determined giving a mean particle diameter $\langle D\rangle=3.1 \mathrm{~nm}$ with a standard deviation $\sigma_{D}=1.0 \mathrm{~nm}$. The core-shell structure cannot be seen in the TEM micrographs due to high $\mathrm{Z}$ value of platinum making it scatter strongly even with sub-nanometer thickness.

The X-ray diffraction pattern for the nanoparticles is shown in Fig. 6.3 with the peaks indexed for platinum with the exception of the peak near $2 \theta \approx 22^{\circ}$ which is attributed to polycrystalline polyvinylpyrrolidone (Saroj, et al., 2013) left over from the synthesis procedure. The location of the peaks for platinum, iron platinum alloy, and $\alpha$-iron are shown underneath the data. No indication of the iron core is seen in the X-ray diffraction pattern as in the reported cases of core-shell nanoparticles of CoO/Pt (Zeleňáková, et al., 2014). The peaks in the X-ray diffraction pattern of the nanoparticles match the locations of the expected peaks for platinum, but the iron-platinum alloy pattern also shown in Fig. 6.3 is very similar because the lattice constants of Pt and FePt are close $(0.392 \mathrm{~nm}$ for Pt and $0.384 \mathrm{~nm}$ for $\mathrm{FePt}$ ). To further verify that the particles formed had a platinum shell rather than an iron-platinum alloy, the high resolution TEM image of Fig 6.2 was analyzed further.

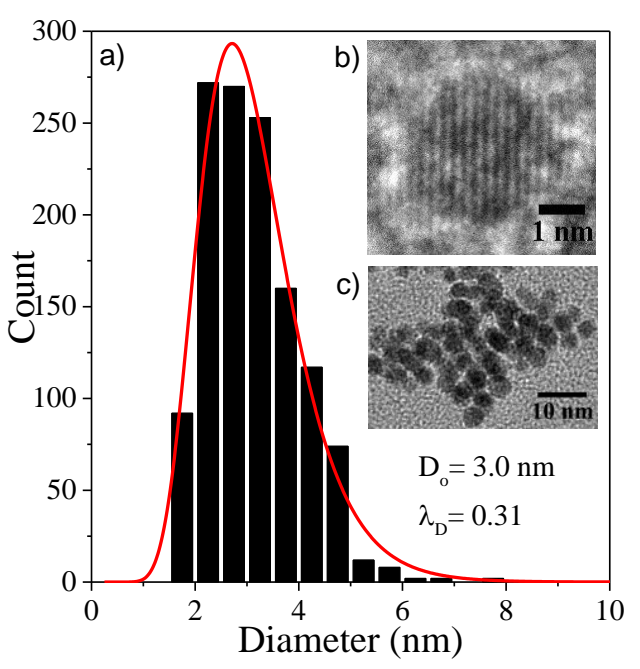

Figure 6.2.: a) Histogram of particle diameters calculated from measured particle cross section areas. Red curve is fit to log-normal distribution. b) High resolution TEM image of Fe-Pt nanoparticle. c) Representative micrograph of Fe-Pt particles. 


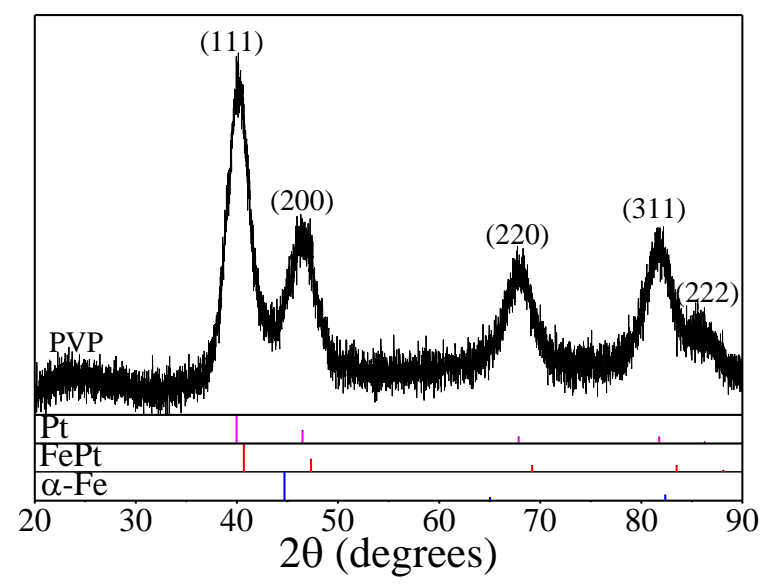

Figure 6.3: XRD data with peaks indexed for Pt. The locations for Pt, FePt alloy, and $\alpha-F e$ peaks are shown below. The broad hump around 23 degrees is attributed to the PVP.

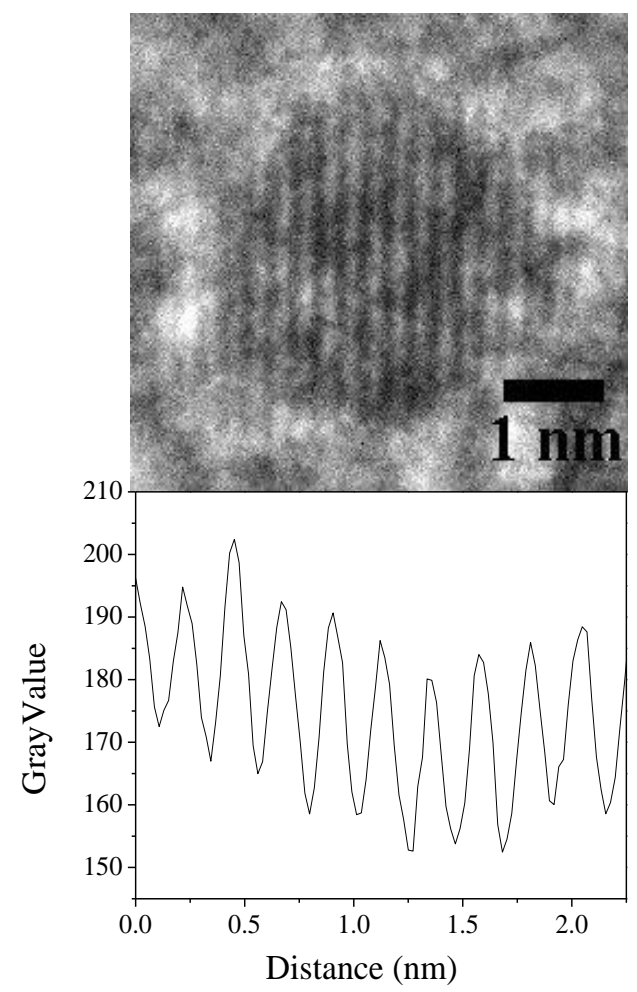

Figure 6.4: top: high resolution transmission electron microscope image of a single particle. The lattice fringes can be clearly seen. bottom: intensity profile from the image used to measure lattice spacing

In Fig. 6.4, the lattice fringes are clearly visible on the high resolution TEM image of a nanoparticle from the sample along with a plot of the average gray value in the micrograph as a function of distance. From the number of cycles per $\mathrm{nm}$, the $\mathrm{d}$ value of $0.226 \mathrm{~nm}$ is calculated. Using the equation:

$$
d_{h k l}=a / \sqrt{h^{2}+k^{2}+l^{2}}
$$


with $a=0.392 \mathrm{~nm}$ and $0.384 \mathrm{~nm}$ for Pt and FePt alloy, respectively, gives $d_{111}=0.226 \mathrm{~nm}$ for Pt and $d_{111}=0.224 \mathrm{~nm}$ for FePt. These results provide confirmation that the outer shell of the nanoparticle is composed of Pt rather than an FePt alloy. From the major Pt peaks in the XRD data, the average crystalline diameter $D_{X R D}$ can be estimated by fitting the data to the Scherrer equation:

$$
D_{X R D}=\frac{0.89 \lambda}{\beta \cos \theta}
$$

where $\lambda=0.154185 \mathrm{~nm}$ is the wavelength of $\mathrm{CuK}_{\alpha}$ radiation used in the experiments, $\beta$ is the peak broadening (in radians), and $2 \theta$ (in radians) is the location of the center of the peak. The calculated value of $D_{X R D}=3.05 \pm 0.20 \mathrm{~nm}$ is in good agreement with the value of $\langle D\rangle=3.1 \mathrm{~nm}$ determined from TEM.

\subsubsection{Thermogravimetric Analysis}

Following the procedure outlined in Chapter 3, thermogravimetric analysis (TGA) was performed after the magnetic measurements to determine the amount of polyvinylpyrrolidone in the sample (Fig. 6.5). Despite rigorous washing with acetone, ethanol, and hexane, TGA indicates that $73 \%$ of the sample mass is due to polyvinylpyrrolidone which contributes only a weak diamagnetic response to the magnetic data. The remaining $27 \%$ of the mass is due to iron-platinum nanostructures. Because both the iron and platinum remain intact at $600{ }^{\circ} \mathrm{C}$, the mass contribution of each must be calculated from the relative amounts of the precursors used in the synthesis.

Iron acetylacetonate has a molecular weight of 353.0 and iron has a molecular weight of 55.845 which means that the $40 \mathrm{mg}$ of iron acetylacetonate used contains $6.328 \mathrm{mg}$ of iron. Likewise, the platinum chloride has a molecular weight of 336.90 while platinum has a molecular weight of 195.09

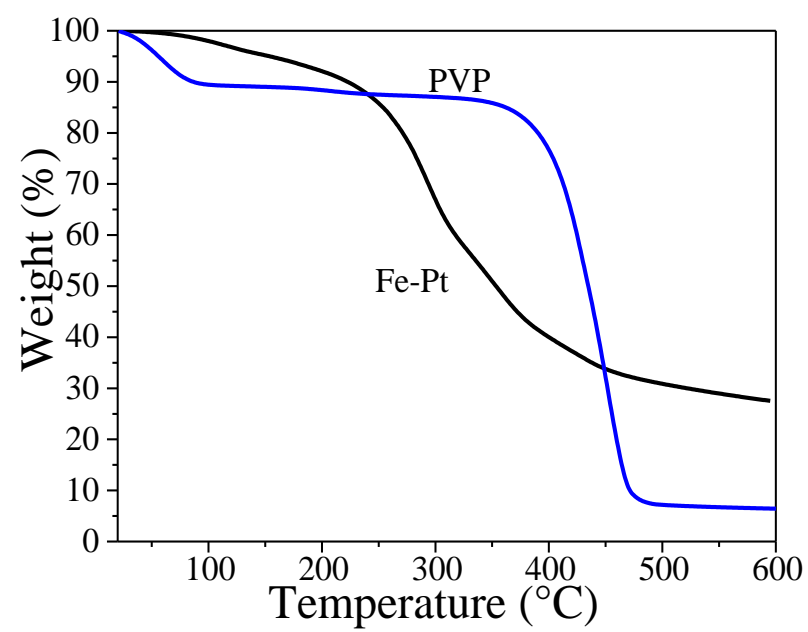

Figure 6.5: TGA results for Fe-Pt sample (black) and pure PVP (blue). From the blue curve, it is clear that any change in weight due to PVP loss should occur before $600{ }^{\circ} \mathrm{C}$. Measurements were made at a temperature ramp rate of $5^{\circ} \mathrm{C} /$ minute. 
which means that the $45 \mathrm{mg}$ of platinum chloride contains $26.055 \mathrm{mg}$ of platinum. So, the mass of the nanoparticles is estimated to be $19.6 \%$ iron which is responsible for the measured magnetization since platinum is only weakly paramagnetic. Therefore, only $5.3 \%$ of the total mass of the sample is from iron and expected to contribute significantly to the magnetization.

Working from the masses of $\mathrm{Fe}$ and $\mathrm{Pt}$ calculated above, the atomic ratio of $\mathrm{Fe}$ to $\mathrm{Pt}$ is found to be 1.18. Assuming the core is $\alpha$-Fe with a lattice constant $a=0.28665 \mathrm{~nm}$ and using the value of $a=0.39242 \mathrm{~nm}$ for FCC Pt, the volume of iron is determined to be $1 / 3$ the volume of platinum in the nanoparticles. Then, for a spherical particle with an outer diameter of $\langle D\rangle=3.1 \mathrm{~nm}$, the diameter of the core $\left(D_{\text {core }}\right)$ can be calculated using

$$
D_{\text {core }}{ }^{3}=\frac{1}{3}\left[\langle D\rangle^{3}-D_{\text {core }}{ }^{3}\right]
$$

to get $D_{\text {core }}=2.0 \mathrm{~nm}$. Alternatively, using the mass ratio of $\mathrm{Fe}$ to $\mathrm{Pt}$ of 0.244 , and the densities $\rho_{F e}=7.874 \mathrm{~g} / \mathrm{cm}^{3}$ and $\rho_{P t}=21.45 \mathrm{~g} / \mathrm{cm}^{3}$ to calculate $D_{\text {core }}$ from

$$
D_{\text {core }}^{3} \rho_{F e}=0.244\left[\langle D\rangle^{3}-D_{\text {core }}{ }^{3}\right] \rho_{P t}
$$

yields $D_{\text {core }}=2.2 \mathrm{~nm}$ (Pisane, et al., 2015). The estimates for the average core diameter calculated in both ways are used later to estimate the expected magnetic moment per particle.

\subsection{Temperature and Magnetic Field Dependence of Magnetization}

\subsubsection{Zero Field-Cooled and Field-Cooled Magnetization}

Following the procedure outlined in Chapter 4, zero field-cooled and field-cooled measurements

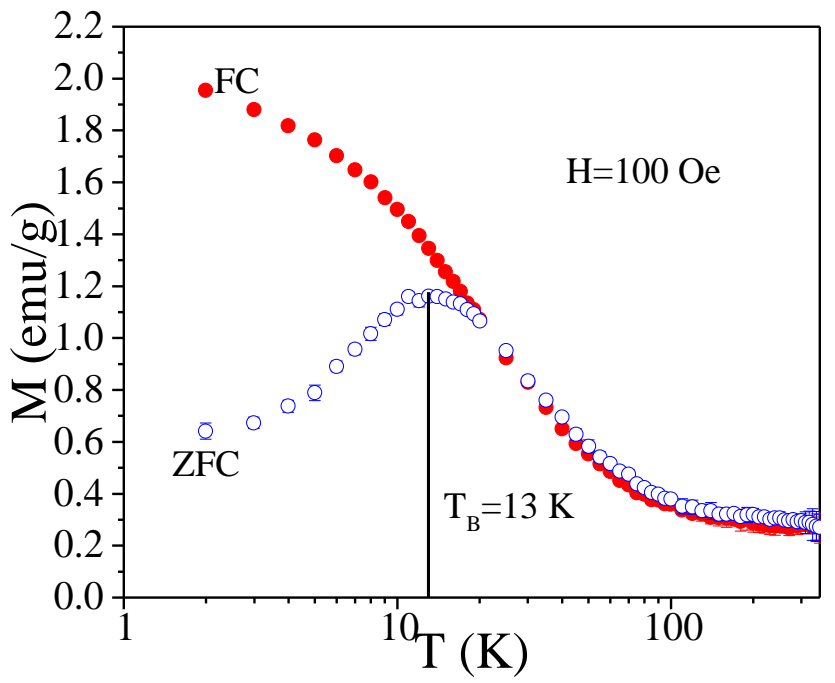

Figure 6.6: Temperature dependence of the field-cooled (solid red circles) and zero-field-cooled (open blue circles) magnetization in a 100 Oe measuring field. 
were performed in a 100 Oe measuring field to find $T_{B}=13.0 \mathrm{~K}$. The broad peak in the ZFC data of Fig. 6.6 occurs because of the distribution in particle sizes present in the sample since smaller particles become unblocked at lower temperatures and larger particles are unblocked at higher temperatures so that the switching from blocked to the unblocked state happens more gradually for a wider distribution of particle sizes.

\subsubsection{Magnetization as a function of Applied Magnetic Field Strength}

The magnetic field dependence of the magnetization of the blocked particles is shown in Fig. 6.7 at $2 \mathrm{~K}$. The value of $M$ was measured for fields up to $\pm 90 \mathrm{kOe}$ and the low field data is shown in more detail in the inset of Fig 6.7. At $2 \mathrm{~K}$, the coercivity $H_{C}=750$ Oe is significantly larger than that observed for FePt alloy nanoparticles of comparable size (Seehra, et al., 2010). The temperature dependence of $H_{C}$

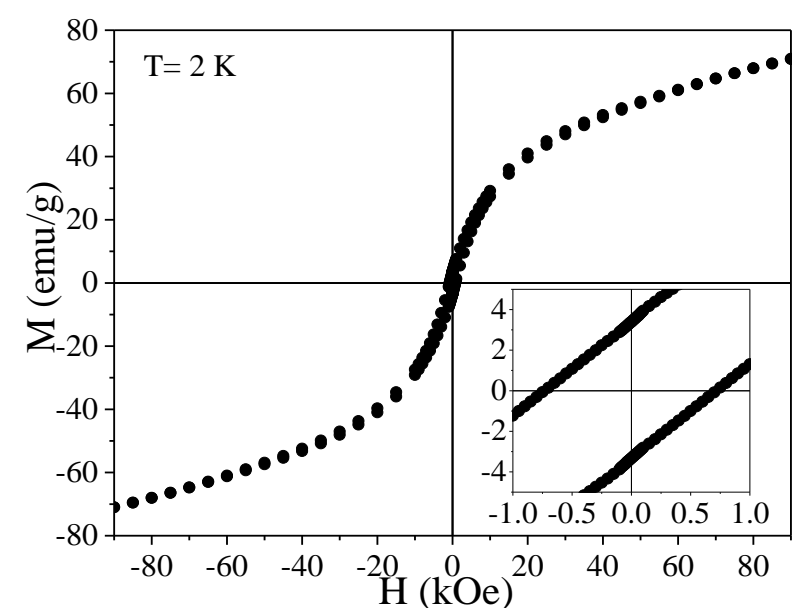

Figure 6.7: Hysteresis loop taken at $2 \mathrm{~K}$. Inset: low-field data expanded to show $\mathrm{H}_{C}=750$ Oe.

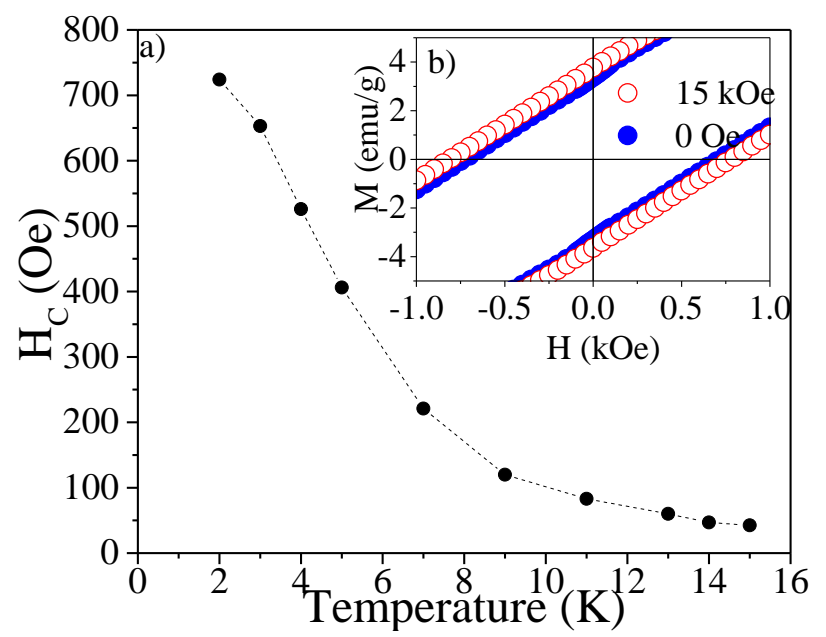

Figure 6.8: a) $H_{C}$ vs. T for ZFC hysteresis measurements b) low field region expanded for both ZFC hysteresis at $2 \mathrm{~K}$ (solid blue circles) and hysteresis loop of same sample cooled in $15 \mathrm{kOe}$ field (open red circles) 


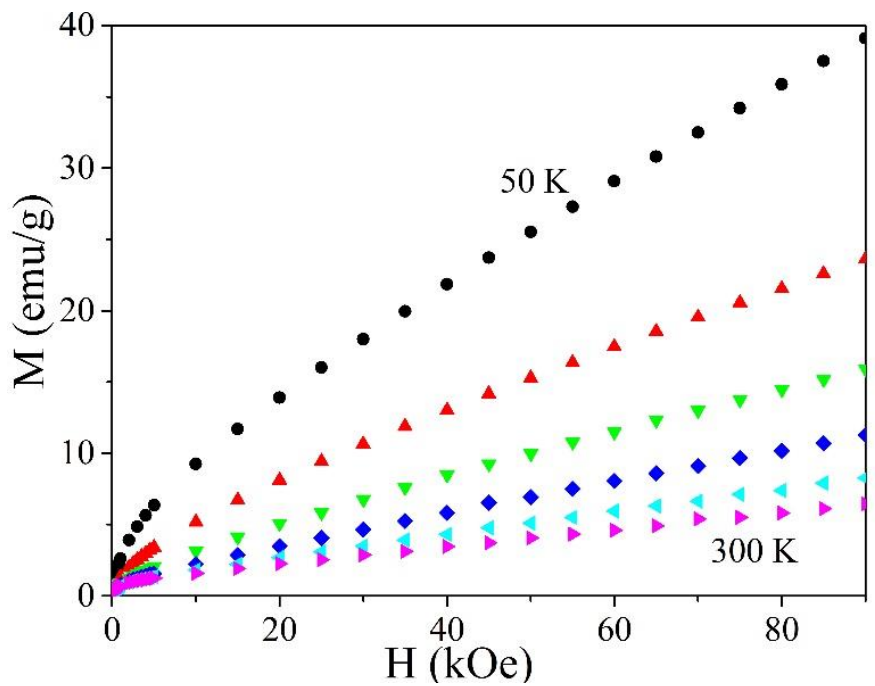

Figure 6.9: $M$ vs. H for Fe-Pt core-shell nanoparticles at $T=50,100,150,200,250$, and $300 \mathrm{~K}$

is shown in Fig. 6.8. Measurements made under the field-cooled condition yielded only slightly increased $H_{C}$ even for a $15 \mathrm{kOe}$ cooling field (Inset of Fig. 6.8). The magnitudes of loop-shift or exchange bias values determined from the hysteresis loops are practically zero within experimental uncertainty $(<20$ Oe).

Above $T_{B}, M$ vs. $H$ from 0 to $90 \mathrm{kOe}$ was measured for $T=50 \mathrm{~K}, 100 \mathrm{~K}, 150 \mathrm{~K}, 200 \mathrm{~K}, 250 \mathrm{~K}$, and $300 \mathrm{~K}$ and the plots of $M$ vs. $H$ are shown in Fig. 6.9. The data were then analyzed using the modified Langevin analysis methods described in Chapters 4 and 5. The results of the analysis are given in section 6.7 .

\subsection{Temperature Dependence of ac Magnetic Susceptibilities}

To determine the relaxation rate and the strength of the interparticle interactions in the system, the temperature dependence of the in-phase and out-of-phase ac magnetic susceptibilities was measured for temperatures between 2 and $20 \mathrm{~K}$ encompassing the blocking temperature at frequencies of $f_{m}=10 \mathrm{~Hz}$, $50 \mathrm{~Hz}, 100 \mathrm{~Hz}, 500 \mathrm{~Hz}, 1 \mathrm{kHz}$, and $5 \mathrm{kHz}$ with an ac magnetic field amplitude $H_{a c}=10$ Oe and no applied dc magnetic field. The $\chi^{\prime \prime}$ data of Fig. 6.10 are noisy and the peaks in the $\chi^{\prime \prime}$ data cannot be clearly determined. Because the in-phase and out-of-phase susceptibilities are related by (Singh, et al., 2009)

$$
\chi^{\prime \prime}=C \frac{\partial\left(T \chi^{\prime}\right)}{\partial T}
$$

where $C$ is a constant, the value of $T_{B}$ used in the analysis of section 6.7 was determined from the location of the peak in $\frac{\partial\left(T \chi^{\prime}\right)}{\partial T}$. 


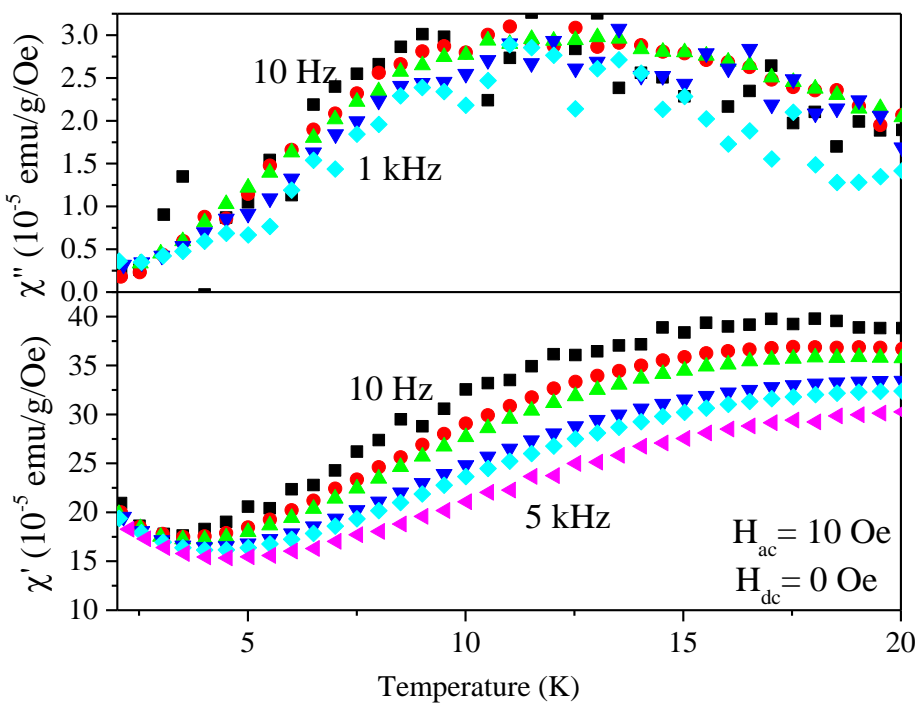

Figure 6.10: Top: Out-of-phase susceptibility vs temperature Bottom: In-phase susceptibility vs. temperature. Data taken at $10 \mathrm{~Hz}, 50 \mathrm{~Hz}, 100 \mathrm{~Hz}, 500 \mathrm{~Hz}, 1 \mathrm{kHz}$, and $5 \mathrm{kHz}$ with an ac magnetic field amplitude of 10 Oe and no applied dc magnetic field.

\subsection{Data Analysis and Interpretation}

\subsubsection{Néel-Brown Relaxation and Interparticle Interactions}

Using the values of $T_{B}$ determined from the peaks in $\partial\left(T \chi^{\prime}\right) / \partial T$ as a function of the measuring frequency $f_{m}, \Phi=0.09$ was calculatd using

$$
\Phi=\frac{\Delta T_{B}}{T_{B} \Delta \log \left(f_{m}\right)} .
$$

Following the discussions in Chapter 4 and 5, this value of $\Phi$ indicates significant interparticle interactions. As was shown in Chapter 5, the variation in $T_{B}$ with $f_{m}$ can be described using the VogelFulcher relationship:

$$
T_{B}=T_{o}+\frac{T_{a}}{\ln \left(f_{o} / f_{m}\right)}
$$

where $T_{o}$ provides a measure of the strength of interparticle interaction. A value of $T_{o}=5 \mathrm{~K}$ was determined to provide the best fit to the data and $\left(T_{B}-T_{o}\right)^{-1}$ vs. $\ln \left(f_{m}\right)$ was plotted (Fig. 6.11) and fit to line to yield $T_{a}=K_{a} V / k_{B}=144 \pm 9 \mathrm{~K}$ and $\ln \left(f_{o}\right)=24.7 \pm 2.1$. The resulting magnitude of $f_{o} \cong 5.3 \times 10^{10}$ $\mathrm{Hz}$ is quite consistent with the value of $f_{o}=2.6 \times 10^{10} \mathrm{~Hz}$ reported in Chapters $4 \& 5$ for $\gamma-\mathrm{Fe}_{2} \mathrm{O}_{3}$ nanoparticles and the value of $f_{o}=1.8 \times 10^{10} \mathrm{~Hz}$ reported for $\mathrm{Ni}$ nanoparticles in $\mathrm{SiO}_{2}$ (Singh, et al., 2009). Using $T_{a}=144 \pm 9 \mathrm{~K}$ and $D_{\text {core }}=2.0$ (2.2) nm yields $K_{a}=4.74 \times 10^{6} \pm 0.30 \times 10^{6} \mathrm{ergs} / \mathrm{cm}^{3}$ $\left(3.56 \times 10^{6} \pm 0.23 \times 10^{6} \mathrm{ergs} / \mathrm{cm}^{3}\right)$, which is an order of magnitude larger than the bulk anisotropy value for 


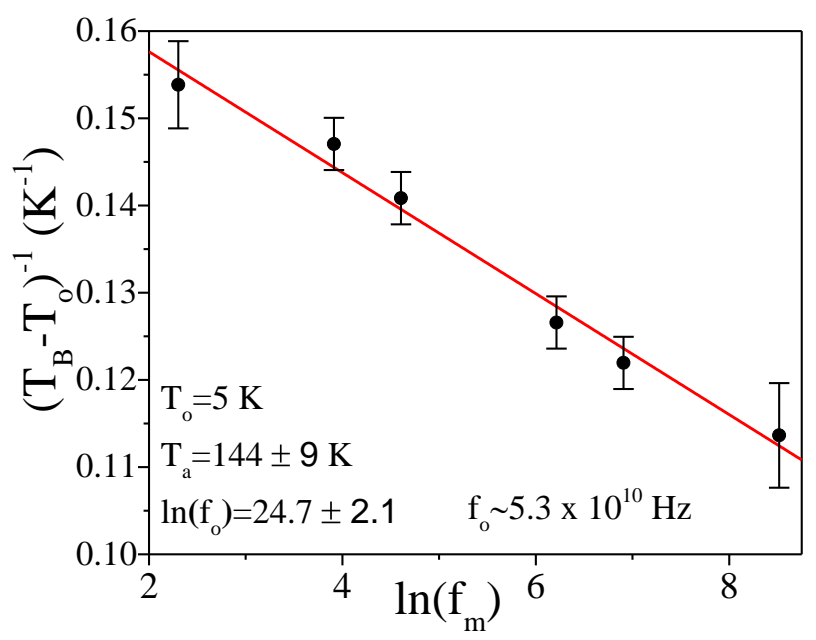

Figure 6.11: Data are $\left(T_{B}-T_{o}\right)^{-1}$ values plotted against $\ln \left(f_{m}\right)$. Red line is a fit to the VogelFulcher equation for $T_{o}=5 \mathrm{~K}$ to yield $T_{a}=144 \pm 9 \mathrm{~K}$ and $\ln \left(f_{o}\right)=24.7 \pm 2.1$.

Fe $\left(K_{a}=4.2 \times 10^{5} \mathrm{erg} / \mathrm{cm}^{3}\right)$. These magnitudes of $K_{a}$ agree well with the $K_{a}=2.5 \times 10^{6} \mathrm{erg} / \mathrm{cm}^{3}$ reported for Fe nanoparticles (Ibusuki, et al., 2001). Evidence of the enhanced value of $K_{a}$ can be seen in Fig. 6.8 in the large $H_{C}$ value due to the proportionality of $K_{a}$ and $H_{C}$.

\subsubsection{Analysis of $M$ vs. $H$ above $T_{B}$}

The interpretation of the variation of the $M$ vs. $H$ data of Fig. 6.9 is considered next. From the plot of $M$ vs. $H / T$ in Fig. 6.12, it is clear that for the Fe-Pt core-shell nanoparticles, only the data at $50 \mathrm{~K}$ and $100 \mathrm{~K}$ scale with $H / T$ whereas the data at higher temperatures do not scale with $H / T$. This is because for lower $H / T$ values, the contribution to $M$ comes from the larger particles only (Ibrahim, et al., 1992) and, due to the width of the distribution in diameters for this sample, $(\sigma=1.0 \mathrm{~nm})$, the effects

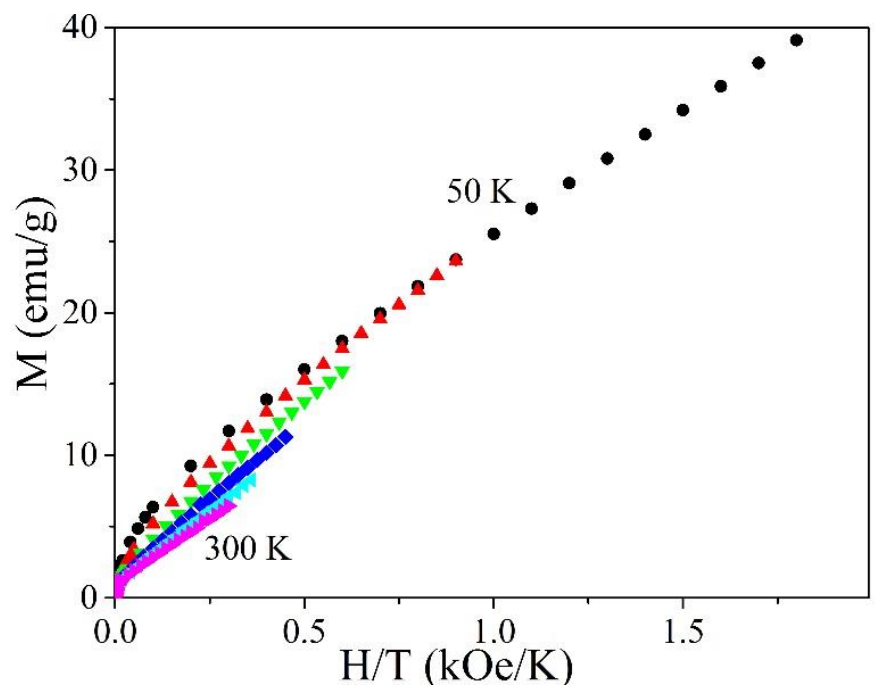

Figure 6.12: $M$ vs. H scaled for temperature. The higher temperature data (150-300 K) appear not to fall along the same line as the 50 \& $100 \mathrm{~K}$ data. 


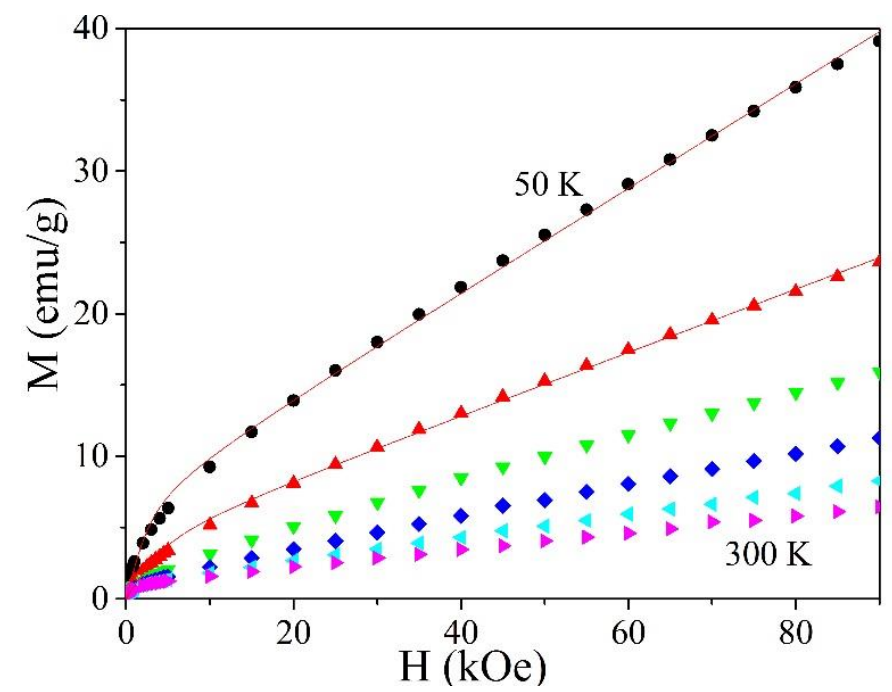

Figure 6.13: Data of Fig. 6.9 showing the fit to the modified Langevin function for $50 \mathrm{~K}$ and $100 \mathrm{~K}$ (solid lines) with fit parameters given in Table 6.1.

Table 6.1:Parameters from the fit of Fe-Pt M vs. H data to Eq. 6.8 with the fits shown in Fig. 6.13

\begin{tabular}{|c|c|c|c|c|}
\hline$T(\mathrm{~K})$ & $\mu_{p}\left(\mu_{B}\right)$ & $M_{o}(\mathrm{emu} / \mathrm{g})$ & $\chi_{a}\left(10^{-4} \mathrm{emu} / \mathrm{g} \mathrm{Oe}\right)$ & $\mathrm{R}^{2}$ \\
\hline 50 & $617 \pm 69$ & $7.04 \pm 0.30$ & $3.65 \pm 0.05$ & 0.99887 \\
\hline 100 & $780 \pm 81$ & $4.21 \pm 0.21$ & $2.20 \pm 0.03$ & 0.99885 \\
\hline
\end{tabular}

become quite apparent. As the temperature is increased, the available magnetic fields cover only smaller values of $H / T$ and so contributions to $M$ are due to larger particles only. Therefore, to obtain values of particle moments that are more representative of the sample as a whole, the results of the fits to the data taken at $50 \mathrm{~K}$ and $100 \mathrm{~K}$ only are discussed.

Temporarily ignoring the distribution in particle sizes, the data taken at $50 \mathrm{~K}$ and $100 \mathrm{~K}$ are fit to a modified Langevin:

$$
M=M_{o} L\left(\frac{\mu_{p} H}{k_{B} T}\right)+\chi_{a} H
$$

This fit, shown in Fig. 6.13, yields $\mu_{p}=617 \mu_{B}$ at $50 \mathrm{~K}$ and $780 \mu_{B}$ at $100 \mathrm{~K}$. All the other parameters of the fit are shown in Table 6.1. Using the $D_{\text {core }}$ values calculated in Section 6.4.2, $a=0.287 \mathrm{~nm}$ for cubic $\mathrm{Fe}$, and a magnetic moment of $4.44 \mu_{B}$ per unit cell, the magnetic moment per particle was estimated using

$$
\frac{\pi}{6}\left(\frac{D_{\text {core }}}{a}\right)^{3} \times 4.44 \mu_{B}
$$

Eq. 6.9 assumes all moments in the iron core are completely aligned. For $D_{\text {core }}=2.0 \mathrm{~nm}(2.2 \mathrm{~nm})$, Eq. 6.9 gives $\mu_{p}=790 \mu_{B}\left(1050 \mu_{B}\right)$. Therefore, the measured magnetic moment is consistent with the calculated moment for an iron core with a $2.0 \mathrm{~nm}$ diameter with all spins aligned. 


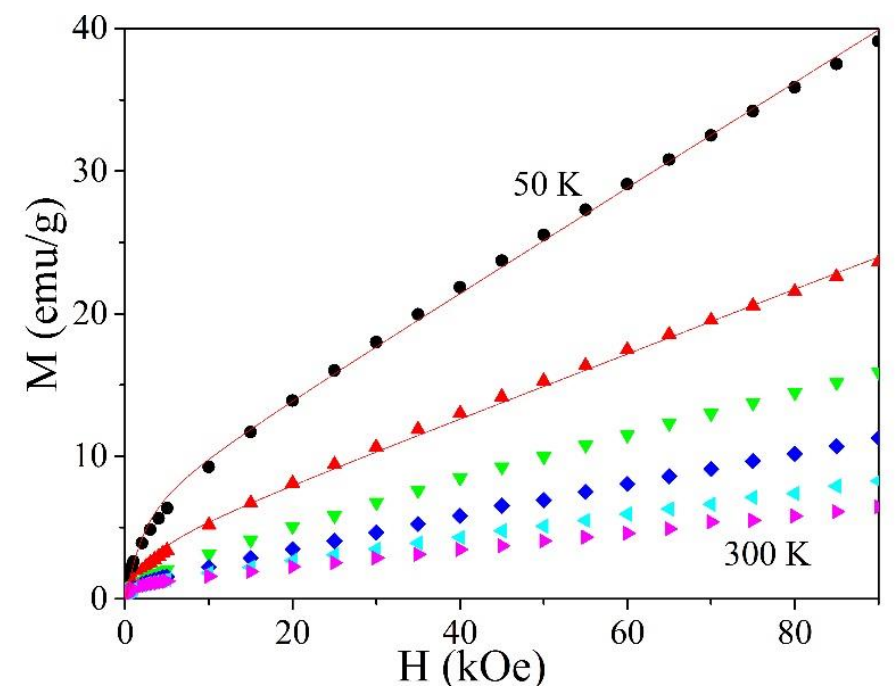

Figure 6.14: Data of Fig. 6.9 showing the fit to a modified Langevin function including a distribution of magnetic moments (solid lines) for $50 \mathrm{~K}$ and $100 \mathrm{~K}$ with fit parameters given in Table 6.2.

Because there is a lognormal distribution of particle diameters, the data have also been fit to a modified Langevin equation that includes a lognormal distribution of particle magnetic moments:

$$
M(H, T)=N \int_{0}^{\infty} \mu \mathcal{L}\left(\frac{\mu H}{k_{B} T}\right) f(\mu) d \mu+\chi_{a} H
$$

following the same procedure as used in Chapters $4 \& 5$. The results of the fits to Eq. 6.10 are shown in Fig. 6.14 with the parameters given in Table 6.2. Fitting the $50 \mathrm{~K}(100 \mathrm{~K})$ data with Eq. 6.10 yields key parameters $\mu_{o}=587 \mu_{B}\left(630 \mu_{B}\right)$ and $s=0.35(0.73)$. From the results in Table $6.2, M_{o}=6.89 \mathrm{emu} / \mathrm{g}$ $(3.72 \mathrm{emu} / \mathrm{g})$ and $\langle\mu\rangle=624 \mu_{B}\left(822 \mu_{B}\right)$ with $\sigma=225 \mu_{B}\left(690 \mu_{B}\right)$ have been calculated. These values are in relatively good agreement with the values from the fit to Eq. 6.8 and illustrate the strong temperature dependence of the magnetic behavior above $T_{B}$ for nanoparticle samples with a distribution of particle diameters. As the temperature is raised from $50 \mathrm{~K}$ to $100 \mathrm{~K}$, the number of particles contributing to the magnetic moment drops by more than half while the average moment of the contributing particles increases and the distribution of the observable moments becomes more skewed as the smallest particles' contributions are removed. Because of this, the distribution of magnetic moments observed above $100 \mathrm{~K}$ is no longer representative of the distribution in particle size illustrated in Fig. 6.2.

Table 6.2: Parameters from the fit of Fe-Pt Mvs. H data to Eq. 6.10 with the fits shown in Fig. 6.14.

\begin{tabular}{|c|c|c|c|c|c|}
\hline$T(\mathrm{~K})$ & $N\left(10^{17} \mathrm{~g}^{-1}\right)$ & $\mu_{o}\left(\mu_{B}\right)$ & $s$ & $\chi_{a}\left(10^{-4} \mathrm{emu} / \mathrm{g} \mathrm{Oe}\right)$ & $\mathrm{R}^{2}$ \\
\hline 50 & $11.9 \pm 3.5$ & $587 \pm 239$ & $0.35 \pm 0.44$ & $3.68 \pm 0.05$ & 0.99897 \\
\hline 100 & $4.88 \pm 2.21$ & $630 \pm 390$ & $0.73 \pm 0.33$ & $2.25 \pm 0.04$ & 0.99887 \\
\hline
\end{tabular}


In Fig 6.14, it is apparent that $M$ does not saturate for magnetic field strength up to $90 \mathrm{kOe}$. This makes extrapolating the values of $M_{o}$ to $T=0 \mathrm{~K}$ difficult as the large linear componenet of $M$ dominates at large fields. The maximum value of $M$ at $2 \mathrm{~K}$ and $90 \mathrm{kOe}$ is $\sim 70 \mathrm{emu} / \mathrm{g}$ which is much lower than the saturation magnetization of bulk iron (215 emu/g). The low value of $M$ compared to bulk as well as the non-saturation of $M$ at $90 \mathrm{kOe}$ are likely due to canting of the magnetic moments in the iron core. Other reported results on iron nanoparticles coated with nonmagnetic material include $4.6 \mathrm{~nm}$ Fe cores coated in Pt with $M_{S} \sim 179 \mathrm{emu} / \mathrm{g}$ at $4 \mathrm{~K}$ (Pana, et al., 2013) and $5 \mathrm{~nm}$ Fe nanoparticles coated with surfactant ( $\beta$ dikeytone) with $M_{S} \sim 210$ at $5 \mathrm{~K}$ (Monson, et al., 2013). Thus, the value of $M_{S}$ likely depends quite strongly on the size of the Fe core.

\subsection{Summary}

In this chapter, the structural and magnetic characterization of Fe-Pt core-shell nanoparticles with an estimated average core diameter of $2.2 \mathrm{~nm}$ have been presented. The observed $T_{B}=13 \mathrm{~K}$ below which $H_{C}$ increases to 750 Oe with decreasing temperature to $2 \mathrm{~K}$. Both the large coercivity and the nonsaturation of $M$ are likely due to the small size of the iron cores for which large portions of the spins may be canted near the surface of the core. The values of $\mu_{p}$ and $\langle\mu\rangle$ determined for lower temperatures above $T_{B}$ are in good agreement with the calculated values for a $2 \mathrm{~nm}$ magnetic Fe core. 


\section{Chapter 7}

\section{Conclusions and Publications}

\subsection{Conclusions}

In this dissertation results of a detailed study of the ac and dc magnetic properties of 4 samples of $\gamma-\mathrm{Fe}_{2} \mathrm{O}_{3}$ nanoparticles of different sizes are presented along with the study of a sample of Fe-Pt core-shell nanoparticles. The structural properties of the nanoparticles have been carefully characterized using transmission electron microscopy, X-ray diffraction, Fourier transform infrared spectroscopy, and thermogravimetric analysis to aid in the analysis of the magnetic behavior. The results of these studies are summarized below.

- Maghemite nanoparticles with mean diameters of $7.0 \mathrm{~nm}, 6.3 \mathrm{~nm}, 3.4 \mathrm{~nm}$, and $2.5 \mathrm{~nm}$ were synthesized with approximate oleic acid coating thickness of $t=1.0 \mathrm{~nm}, 1.3 \mathrm{~nm}, 0.8 \mathrm{~nm}$, and $0.5 \mathrm{~nm}$, respectively and their key properties are summarized here (reproduction of Table 5.13):

\begin{tabular}{|c|c|c|c|c|c|c|}
\hline$\langle D\rangle(\mathrm{nm})$ & $\sigma_{D}(\mathrm{~nm})$ & $\mathrm{T}_{\mathrm{B}}(\mathrm{K})$ & $\mathrm{T}_{\mathrm{o}}(\mathrm{K})$ & $\mathrm{T}_{\mathrm{B}}-\mathrm{T}_{\mathrm{o}}(\mathrm{K})$ & $\mathrm{K}_{\mathrm{a}}\left(10^{5} \mathrm{emu} / \mathrm{cm}^{3}\right)$ & $\Phi$ \\
\hline 2.5 & 0.7 & 29 & 12.5 & 16.5 & $79.95 \pm 28$ & $0.080 \pm 0.003$ \\
\hline 3.4 & 0.7 & 21 & 2.5 & 18.5 & $18.57 \pm 10$ & $0.094 \pm 0.001$ \\
\hline 6.3 & 0.6 & 42 & 11 & 31 & $7.51 \pm 1.01$ & $0.084 \pm 0.001$ \\
\hline 7.0 & 0.8 & 35 & 0 & 35 & $5.57 \pm 1.80$ & $0.120 \pm 0.013$ \\
\hline
\end{tabular}

- The crystalline sizes determined from X-ray diffraction for the maghemite nanoparticles are consistent with the mean diameters determined from transmission electron micrographs providing evidence for the high degree of crystallinity of the nanoparticles. The oleic acid on the surface of the particles forms unidentate, bidentate, and bridging bonds with the iron at the surface as determined by Fourier transform infrared spectroscopy;

- From the analysis of the variation of $T_{B}$ with respect to $f_{m}$, the absence of significant interparticle interaction and the Néel-Brown attempt frequency of $2.6 \times 10^{10} \mathrm{~Hz}$ has been inferred for the $7.0 \mathrm{~nm}$ maghemite nanoparticles;

- The absence of any notable coercivity $H_{C}$ observed in the $7.0 \mathrm{~nm}, 3.4 \mathrm{~nm}$, and $2.5 \mathrm{~nm}$ maghemite nanoparticles is different than the large values reported in maghemite nanoparticle samples prepared by different methods indicating that the synthesis route may influence the magnetic properties of $\gamma$ $\mathrm{Fe}_{2} \mathrm{O}_{3}$ nanoparticles;

- The average magnetic moment per particle determined using a modified Langevin function that ignores a distribution in particle magnetic moments yields results consistent with those obtained by considering a distribution of magnetic moments so long as the width parameter $s$ is significantly 
below 0.83 . Therefore fitting to a modified Langevin equation that does not include a distribution of magnetic moments is not advisable for values of $s>0.83$;

- The mathematical relationship between $T_{o}$ and $\Phi$ has been derived and the theoretical basis for the value of $\Phi_{o} \approx 0.11$ to 0.15 often observed experimentally has been presented. In addition, data from the literature has been used to verify the relationships between $T_{o}$ and $\Phi$;

- The trend in increase in magnetic anisotropy constant $K_{a}$ with decrease in nanoparticle diameter $D$ observed for $\gamma-\mathrm{Fe}_{2} \mathrm{O}_{3}$ nanoparticles, when careful consideration is given to the effects of interparticle interactions, is observed. However the variation is more rapid than the $1 / D$ variation expected from the commonly used relation: $K=K_{\text {bulk }}+\frac{6 K_{\text {surface }}}{D}$;

- The structural and magnetic characterization of Fe-Pt core-shell nanoparticles with an estimated average core diameter of $2.2 \mathrm{~nm}$ have been carried out following the methodology of Chapters $3 \& 4$ to find $T_{B}=13 \mathrm{~K}$ below which $H_{C}$ increases to $750 \mathrm{Oe}$ with decreasing temperature to $2 \mathrm{~K}$. The large coercivity and the non-saturation of $M$ in the Fe-Pt nanoparticles are likely due to the small size of the iron cores for which large portions of the spins may be canted near the surface of the core. The values of $\mu_{p}$ and $\langle\mu\rangle$ determined for lower temperatures above $T_{B}$ for the Fe-Pt nanoparticles are in good agreement with the calculated values for a $2 \mathrm{~nm}$ magnetic Fe core.

\subsection{Publications \& Presentations}

\subsubsection{Publications based on this dissertation:}

- $\quad$ K. L. Pisane, E. C. Despeaux, \& M. S. Seehra, "Magnetic relaxation and correlating effective magnetic moment with particle size distribution in maghemite nanoparticles" J. Magn. Magn. Mater. 384, 148-154 (2015).

- $\quad$ K. L. Pisane, Sobhit Singh, \& M. S. Seehra, "Synthesis, structural characterization and magnetic properties of Fe/Pt core-shell nanoparticles" J. Appl. Phys. 117, 17D708 (2015).

- M. S. Seehra \& K. L. Pisane, "Relationship between blocking temperature and strength of interparticle interaction in magnetic nanoparticle systems"-under review J. Magn. Magn. Mater. (Letter).

- K. L. Pisane \& M.S. Seehra, "Size dependence of magnetic anisotropy and interparticle interactions in maghemite nanoparticles", Manuscript under preparation. 


\subsubsection{Other publications}

- Z. Wang, K. L. Pisane, \& M. S. Seehra "Magnetic Determination of the Electronic State of Cu and Exchange Interactions in the $\alpha$ - and $\beta$-phases of Molecular Semiconductor Copper Phthalocyanine (C32H16N8Cu)" IEEE Trans. Magn.-Conf. (2015) in press

- $\quad$ K. M. Dunnick, R. Pillai, K. L. Pisane, A. B. Stefaniak, E. M. Sabolsky \& S. S. Leonard "The Effect of Cerium Oxide Nanoparticle Valence State on Reactive Oxygen Species and Toxicity” Biol. Trace Elem. Res.166, 96 (2015).

- P. Fraundorf, K. Pisane, \& R. Collins "Zero-loss/deflection map analysis" Microscopy and Microanalysis 16 (S2), 1534-1535 (2010).

\subsubsection{Presentations}

- InterMag 2015 "Magnetic Determination of the Electronic State of Copper in the Molecular Semiconductor Copper Phthalocyanine $\left(\mathrm{C}_{32} \mathrm{H}_{16} \mathrm{~N}_{8} \mathrm{Cu}\right)$ " Beijing China, May 2015 (poster);

- 59th Annual Magnetism \& Magnetic Materials Conference "Synthesis, structure and magnetic properties of Fe@Pt core-shell nanoparticles” Honolulu HI, Nov 72014 (podium presentation);

- Inaugural APS Mid-Atlantic Section Meeting "Effects of Particle Size on the Magnetic Properties of Maghemite Nanoparticles" State College PA, Oct 42014 (podium presentation);

- 2014 NanoSAFE Bioelectronics and Biosensing International Symposium "Synthesis and Magnetic Characterization of Maghemite Nanoparticles Designed for Targeted Cancer Therapy" Morgantown WV, April 272014 (poster);

- APS March Meeting “Synthesis and Magnetic Characterization of Maghemite Nanoparticles Designed for Targeted Cancer Therapy” Denver CO, March 42014 (poster);

- APS March Meeting "On the Cubic Phase Stability and Magnetic Properties of Cu-doped ZrO2" Denver CO, March 42014 (poster);

- Eighteenth Annual Meeting of the NASA-Missouri Space Grant Consortium "Unlayered Graphene in Presolar Graphene Onions", Columbia MO, April 17th, 2009; 


\section{Appendix: ACMS Measurement Sequences}

\section{Temperature Approach Methods used:}

1. Fast Settle: approach the targeted value(s) quickly, the temperature may overshoot the target slightly before reaching the targeted value

2. No O'shoot: approach the targeted value more slowly than fast settle, the temperature will not overshoot the target value, but the final approach will take much longer than the fast settle approach

\section{Field Approach Methods used:}

1. Linear: magnetic field approaches the targeted value from the current value directly

2. Oscillate: magnetic field approaches the targeted value $(0 \mathrm{Oe})$ by oscillating. e.g. if the field is 500 Oe and it is set to 0 Oe using the oscillate approach it will first go to a value $\sim-450$ Oe, then back to a positive value, etc. The purpose of the oscillate approach is to remove any remnant magnetization in the sample or magnet while setting the field to zero.

ac Measurement Sequence

Set Magnetic Field 0.00e at 100.00e/sec, Oscillate, Persistent

Wait For Field, Delay 0 secs, No Action

Set Temperature $10 \mathrm{~K}$ at $12 \mathrm{~K} / \mathrm{min}$. Fast Settle

Wait For Temperature, Delay 3600 secs, No Action

Set Temperature $2 \mathrm{~K}$ at $12 \mathrm{~K} / \mathrm{min}$. Fast Settle

Wait For Temperature, Delay 180 secs, No Action

Set Magnetic Field 300.0Oe at 100.0Oe/sec, Linear, Persistent

Wait For Field, Delay 0 secs, No Action

ACMS Locate at 5 Oe $1000 \mathrm{~Hz}$

Scan Temp from $2 \mathrm{~K}$ to $120 \mathrm{~K}$ at $12 \mathrm{~K} / \mathrm{min}$, in 60 steps, Uniform, Fast

AC Mag 5 Oe 10, 20,50,100,200,500,1000,2000, 5000 Hz 1 Sec Stickyrange Flags=33554439

End Scan

Scan Temp from $125 \mathrm{~K}$ to $200 \mathrm{~K}$ at $12 \mathrm{~K} / \mathrm{min}$, in 16 steps, Uniform, Fast

AC Mag 5 Oe 10, 20,50,100,200,500,1000,2000, 5000 Hz 1 Sec Stickyrange Flags=33554439 
End Scan

Set Temperature $300 \mathrm{~K}$ at $20 \mathrm{~K} / \mathrm{min}$. Fast Settle

Set Magnetic Field 0.0Oe at 100.0Oe/sec, Oscillate, Persistent

Wait For Temperature, Field, Delay 3600 secs, No Action

Chain Sequence C:\QdPpms\Data|Kelly-SeehralSPION 2 to 1 Dec 2014\2K_Hysteresis.seq

\section{dc Measurement Sequence: Hysteresis}

Set Temperature $10 \mathrm{~K}$ at $12 \mathrm{~K} / \mathrm{min}$. Fast Settle

Wait For Temperature, Delay 3600 secs, No Action

Set Temperature $2 \mathrm{~K}$ at 20K/min. Fast Settle

Wait For Temperature, Delay 300 secs, No Action

Scan Field from 90000.00 e to 15000.00 at 100.00 e/sec, in 16 steps, Uniform, Linear, Persistent

DC Mag 5 Scans Stickyrange Flags=33554439

End Scan

Scan Field from 10000.0Oe to 5000.0Oe at 100.00e/sec, in 6 steps, Uniform, Linear, Persistent DC Mag 5 Scans Stickyrange Flags=33554439

End Scan

Scan Field from 4000.00e to 1000.00 at 100.00 e/sec, in 16 steps, Uniform, Linear, Persistent DC Mag 5 Scans Stickyrange Flags=33554439

End Scan

Scan Field from 975.00 e to 100.00 at 100.00 e/sec, in 36 steps, Uniform, Linear, Persistent DC Mag 5 Scans Stickyrange Flags=33554439

End Scan

Scan Field from 90.00e to -90.00 at 100.00 e/sec, in 19 steps, Uniform, Linear, Persistent DC Mag 5 Scans Stickyrange Flags=33554439

End Scan

Scan Field from -100.00 e to -975.00 at 100.00 e/sec, in 36 steps, Uniform, Linear, Persistent DC Mag 5 Scans Stickyrange Flags=33554439 
End Scan

Scan Field from -1000.00 e to -4000.00 at 100.00 e/sec, in 16 steps, Uniform, Linear, Persistent DC Mag 5 Scans Stickyrange Flags=33554439

End Scan

Scan Field from -5000.0Oe to -10000.0Oe at 100.00e/sec, in 6 steps, Uniform, Linear, Persistent DC Mag 5 Scans Stickyrange Flags=33554439

End Scan

Scan Field from -15000.00 e to -90000.00 at 100.00 e/sec, in 16 steps, Uniform, Linear, Persistent DC Mag 5 Scans Stickyrange Flags=33554439

End Scan

Scan Field from -85000.0 Oe to -10000.0 Oe at 100.0 Oe/sec, in 16 steps, Uniform, Linear, Persistent DC Mag 5 Scans Stickyrange Flags=33554439

End Scan

Scan Field from -9000.00 e to -5000.00 at 100.00 e/sec, in 5 steps, Uniform, Linear, Persistent DC Mag 5 Scans Stickyrange Flags=33554439

End Scan

Scan Field from -4000.00 e to -1000.00 at 100.00 e/sec, in 16 steps, Uniform, Linear, Persistent DC Mag 5 Scans Stickyrange Flags=33554439

End Scan

Scan Field from -975.00 e to -100.00 at 100.00 e/sec, in 36 steps, Uniform, Linear, Persistent DC Mag 5 Scans Stickyrange Flags=33554439

End Scan

Scan Field from -90.0 Oe to 90.00 at 100.00 e/sec, in 19 steps, Uniform, Linear, Persistent DC Mag 5 Scans Stickyrange Flags=33554439

End Scan

Scan Field from 100.0Oe to 975.00 at 100.00 e/sec, in 36 steps, Uniform, Linear, Persistent DC Mag 5 Scans Stickyrange Flags=33554439

End Scan

Scan Field from 1000.00 e to 4000.00 at 100.00 e/sec, in 16 steps, Uniform, Linear, Persistent DC Mag 5 Scans Stickyrange Flags=33554439 


\section{End Scan}

Scan Field from 5000.0Oe to 10000.00 at 100.00 e/sec, in 6 steps, Uniform, Linear, Persistent DC Mag 5 Scans Stickyrange Flags=33554439

\section{End Scan}

Scan Field from 15000.0 Oe to 90000.00 at 100.00 e/sec, in 16 steps, Uniform, Linear, Persistent DC Mag 5 Scans Stickyrange Flags=33554439

End Scan

Set Magnetic Field 1500.0Oe at 100.0Oe/sec, Linear, Persistent

Wait For Field, Delay 0 secs, No Action

Set Magnetic Field 0.0Oe at 100.0Oe/sec, Oscillate, Persistent

Set Temperature $200 \mathrm{~K}$ at $12 \mathrm{~K} / \mathrm{min}$. Fast Settle

Wait For Temperature, Delay 1800 secs, No Action 


\section{References}

Alayoglu, S., Nilekar, A. U., Mavrikakis, M. \& Eichhorn, B. Nat. Mater. 7, 333 (2008).

Allia, P., Coisson, M., Tiberto, P. Vinai, F., Knobel, M., Novak, M. A. \& Nunes, W. C. Phys. Rev B. 64, 144420 (2001).

Andersson, J.-O., Djurberg, C., Jonsson, T., Svedlindh, P., \& Nordblad, P. Phys. Rev. B 56, 13983 (1997).

Bartolomé, J., García, L. M., Bartolomé, F., Luis, F., López-Ruiz, R, Petroff, F., Deranlot, C, Wilhelm, F. Rogalev, A., Bencok, P., Brookes, N. B., Ruiz, L. \& González-Calbet, J. M. Phys. Rev. B 77, 184420 (2008).

Berkowitz, A. E., Schuele, W. J. \& Flanders, P. J. J. Appl. Phys. 39, 1261(1968).

Berry, C. C. \& Curtis, A. S. G. J. Phys. D: Appl. Phys. 36, R 198 (2003).

Birks, J. B. Proc. Phys. Soc. 63, 65 (1950).

Bødker, F., Mørup, S., \& Linderoth, S. Phys. Rev. Lett. 72, 282 (1994).

Carpenter, E. E. J. Magn. Magn. Mater. 225, 17 (2001).

Chantrell, R. W., Walmsley, N., Gore, J. \& Maylin, M. Phys. Rev. B. 3, 024410 (2000).

Chaudhuri, R. G. \& Paria, S. Chem. Rev. 112, 2373 (2012).

Coey, J. M. D. Phys. Rev. Lett. 27, 1140 (1971).

Demchenko, P., Nedelko, N., Mitina, N., Lewińska, S., Dłużewski, P., Greneche, J. M., Ubizskii, S., Navrotskyi, S., Zaichenko, A. \& Ślawska-Waniewska, A. J. Magn. Magn. Mater. 379, 28 (2015).

Dorman, J. L., Bessais, L. \& Fiorani, D. J. Phys. C 21, 2015 (1988).

Dutta, P., Manivannan, A., Seehra, M. S., Shah, N. \& Huffman, G. P. Phys. Rev. B 70, 174428 (2004).

Fiorani, D. (Ed.), Surface Effects in Magnetic Nanoparticles, New York, NY: Springer (2005).

Fiorani, D., Testa, A. M., Lucari, F., D'Orazio, F. \& Romero, H. Physica B 320, 122 (2002).

Fiorani, D., Viticoli, S., Dormann, J. L., Tholence, J. L. \& Murani, A. P. Phys. Rev. B 30, 2776 (1984).

Gandhi, H. S., Graham, G. W. \& McCabe, R. W. J. Catal. 216, 433 (2003).

Gazeau, F., Bacri, J. C., Gendron, F., Perzynski, R., Raikher, Y. L., Stapanov, V. I. \& Dubois, E. J. Magn. Magn. Mater. 186, 175 (1998).

Grau-Crespo, R., Al-Baitai, A. Y., Saadoune, I., \& De Leeuw, N. H. J. Phys.: Condens. Matter. 22, 255401 (2010).

Gubin, S. P. (Ed.). Magnetic Nanoparticles, Weinheim, Germany: Wiley-VCH. (2009).

Hyeon, T., Le, S. S, Park, J., Chung, Y. \& Na, H. B. J. Am. Chem. Soc. 123, 12798 (2001).

Ibrahim, M. M., Zhao, J. \& Seehra, M. S. J. Mater. Res. 7, 1856 (1992).

Ibusuki, T., Kojima, S., Kitakami, O. \& Shimada, Y. IEEE Trans. Magn. 37, 2223 (2001).

Kalele, S., Gosavi, S. W., Urban, J. \& Kulkarni, S. K. Curr. Sci. 91, 1038 (2006).

Kechrakos, D. \& Trohidu, K. N. Phys. Rev. B 58, 12169 (1998). 
Kittel, C. Introduction to Solid State Physics, New York: Wiley (1976).

Kneller, E. F. \& Luborsky, F. E. J. Appl. Phys. 34, 656 (1963).

Laha, S. S., Tackett, R. J. \& Lawes, G. Physica B: Cond. Matter 448, 69, (2014).

Lu, A.-H., Salabas, E. L. \& Schüth, F. Agnew. Chem. Int. Ed. 46, 1222 (2007).

Lu, L., Zhang, W., Wang, D., Xu, X., Miao, J. \& Jiang, Y. Mater. Lett. 64, 1732 (2010).

Lundgren, L, Svendlindh, P, \& Beckman, O. J. Magn. Magn. Mater. 25, 33 (1981).

Makhlouf, S. A., Parker, F. T. \& Berkowitz, A. E. Phys. Rev. B 55, R14717 (1997).

Millan, A., Urtizberea, A., Silva, N. J. O., Palacio, F., Amaral, V. S., Snoeck, E. \& Serin, V. J. Magn. Magn. Mater. 312, L5 (2007).

Monson, T. C., Venturini, E. L., Petkov, V., Ren, Y., Lavin, J. M. \& Huber, D. L. J. Magn. Magn. Mater. 331, 156 (2013).

Morales, M. P., Veintemillas-Verdaguer, S., Montero, M. I., Serna, C. J., Roig, A., Casas, L. L., Martinez, B. \& Sandiumenge, F. Chem. Mater. 11, 3058 (1999).

Morrish, A. H. The Physical Principles of Magnetism. New York: John Wiley \& Sons. (1955).

Morrish, A. H. \& Haneda, K., J. Magn. Magn. Mater. 35, 105 (1983).

Mørup, S. Europhys. Lett. 28, 671 (1994).

Nakamoto, K. Infrared and Raman Spectra of Inorganic and Coordination Compunds, Fourth Edition New York: John Wiley \& Sons, pp. 231-233 (1986).

Nadeem, K., Krenn, H., Traussnig, T., Würschum, R., Szabó, D. V., \& Letofsky-Papst, I. J. Magn. Magn. Mater. 323, 1998 (2011).

Néel, L. Ann. Geophys. 5, 99 (1949).

Néel, L. Adv. Phys., 4, 191 (1955).

Pana, O., Leostean, C., Soran, M. L., Stefan, M., Macavei, S., Gutoiu, S., Pop, V. \& Chauvet, O. J. Alloys Compds. 574, 477 (2013).

Pankhurst, Q. A., Connolly, J., Jones, S. K., \& Dobson, J. J. Phys. D: Appl. Phys. 36, R167 (2003).

Pankhurst, Q. A., \& Pollard, R. J. Phys. Rev. Lett., 67, 248 (1991).

Pankhurst, Q. A., Thanh, N. T., Jones, S. K., \& Dobson, J. J. Phys. D: Appl. Phys. 42, 224001 (2009).

Pappa, C., Hammond, J., Johanna, G. \& Jacoboni, C. J. Phys. C: Solid St. Phys. 18, 2817 (1985).

Park, J.-I. \& Cheon, J. J. Am. Chem. Soc. 123, 5743 (2001).

Parker, F. T., Foster, M. W., Margulies, D. T. \& Berkowitz, A. E. Phys. Rev. B 47, 7885 (1993).

Pérez, N., Guardia, P., A. G. Roca, Morales, M. P., Serna, C. J., Iglesias, O., Bartolomé, F., García, F. M., Batlle, X. \& Labarta, A. Nanotechnology 19, 475704 (2008).

Pisane, K. L., Despeaux, E. C. \& Seehra, M. S. J. Magn. Magn. Mater. 384, 148 (2015).

Pisane, K. L., Singh, Sobhit \& Seehra, M. S. J. Appl. Phys. 117, 17D708 (2015). 
Punnoose, A., Phanthavady, T., Seehra, M. S., Shah, N., \& Huffman, G. P. Phys. Rev. B 69, 054425 (2004).

Punnoose, A., Seehra, M. S., van Tol, J. \& Brunel, L. C. J. Magn. Magn. Mater. 288, 168 (2005).

Rall, J. D., Seehra, M. S. \& Choi, E. S. Phys. Rev. B 82, 184403 (2010).

Respaud, M., Broto, J. M., Rakoto, H., Fert, A. R., Thomas, L., Barbara, B., Verelst, M., Snoeck, E., Lecante, P., Mosset, A., Osuna, J., Ely, T. O., Amiens, C. \& Chaudret, B. Phys. Rev. B 57, 2925 (1998).

Richardson, J. T. \& Desai, P. J. Catal. 42, 294 (1976).

Saroj, A. L., Singh, R. K. \& Chandra, S. Mater. Sci. Eng. B 178, 231 (2013).

Seehra, M. S. \& Punnoose, A Phys. Rev. B 64, 132410 (2001).

Seehra, M. S., Shim, H., Dutta, P., Manivannan, A. \& Bonevich, J. J. Appl. Phys. 97, 10J509 (2005).

Seehra, M. S., Singh, V., Dutta, P., Neeleshwar, S., Chen, Y. Y., Chen, C. L., Chou, S. W. \& Chen, C. C. J. Phys. D: Appl. Phys. 43, 145002 (2010).

Seehra, M. S., Singh, V., Song, X., Bali, S. \& Eyring, E. M. J. Phys. Chem. Solids 71, 184403 (2010).

Serna, C. J., Bødker, F., Mørup, S., Morales, M. P., Sandiumenge, F., \& Veintemillas-Verdaguer, S. Solid State Commun., 118, 437 (2001).

Shim, H., Dutta, P., Seehra M. S. \& Bonevich, J. Solid St. Commun. 145, 192 (2008).

Shim, H., Mannivannan, A., Seehra, M. S., Reddy, K. M., \& Punnoose, A. J. Appl. Phys. 99, 08Q503 (2006).

Shtrikman, S. \& Wohlfarth, E. P. Phys. Lett. 85A, 467 (1981).

Silva, N. J. O., Amaral, V. S. \& Carlos, L. D. J. Appl. Phys. 100, 054301 (2006).

Singh, V., Seehra, M. S., Bali, S., Eyring, E. M., Shah, N., Huggins, F. E. \& Huffman, G. P. J. Phys. Chem. Solids 72, 1373 (2011).

Singh, V., Seehra, M. S. \& Bonevich, J. J. Appl. Phys. 105, 07 B518 (2009).

Singh, V., Seehra, M. S., Huggins, F. E., Shah, N. \& Huffman, G. P. J. Appl. Phys. 109, 07B506 (2011).

Smart, J. S. Effective Field Theories of Magnetism, Philadelphia \& London: W. B. Saunders Co. (1966).

Soler, M. A. G, Alcantara, G. B., Soares, F. Q., Viali, W. R., Sartoratto, P. P. C., Fernandez, J. R. L., da Silva, S. W., Garg, V. K., Oliveria, A. C. \& Morais, P. C. Surface Science 601, 3921 (2007).

Thanh, N. T. (Ed.). Magnetic Nanoparticles: From Fabrication to Clinical Applications. Boca Raton, FL: CRC Press (2012).

Tholence, J. L. Solid State Commun. 88, 917 (1993).

Tiwari, S. D. \& Rajeev, K. P. Solid St. Commun. 152, 1080 (2012).

Vaishnava, P. P., Senaratne, U., Buc, E. C., Naik, R., Naik, V. M., Tsoi, G. M., \& Wenger, L. E. Phys. Rev. B 76, 024413 (2007). 
Vallejo-Fernandez, G., Whear, O., Roca, A. G., Hussain, S., Timmis, J., Patel, V. \& O’ Grady, K. J. Phys. D: Appl. Phys. 46, 312001 (2013).

Verwey, E. J., \& Heilmann, E. L. J. Chem. Phys. 15, 174 (1947).

Vincent, E., Hammann, J. \& Alba, M. Solid St. Commun. 58, 57 (1986).

Wang, C., Ravi, S., Martinez, G. V., Chinnasamy, V., Raulji, P., Howell, M., Davis, Y., Mallela, J., Seehra, M. S. \& Mohapatra, S. J. Control. Release 163, 82 (2012).

Xiao, G., Liou, S. H., Levy, A., Taylor, J. N. \& Chien, C. L. Phys. Rev. B 34, 7573 (1986).

Zeleňáková, A., Zeleňák, V., Michalík, Š., Kováč, J. \& Meisel, M. W. Phys. Rev. B 89, 104417 (2014).

Zhou, S., Varughese, B., Eichhorn, B., Jackson, G. \& McIlwrath, K. Angew. Chem. Int. Ed. 44, 4539 (2005). 